\title{
Aß42 oligomers trigger synaptic loss through CAMKK2-AMPK-dependent effectors coordinating mitochondrial fission and mitophagy
}

Annie Lee ${ }^{1,2,4 \#}$, Chandana Kondapalli ${ }^{1,2 \#}$, Daniel M. Virga ${ }^{1,2,6}$, Tommy L. Lewis Jr. ${ }^{1,2}$, So Yeon Koo ${ }^{5,7}$, Archana Ashok ${ }^{5,7}$, Georges Mairet-Coello ${ }^{8}$, Sebastien Herzig ${ }^{9}$, Reuben Shaw ${ }^{9}$, Andrew Sproul ${ }^{5,7}$ and Franck Polleux ${ }^{1,2,3 *}$

${ }^{1}$ Department of Neuroscience, Columbia University Medical Center New York, NY

${ }^{2}$ Mortimer B. Zuckerman Mind Brain Behavior Institute, New York, NY

${ }^{3}$ Kavli Institute for Brain Sciences, Columbia University Medical Center, New York, NY

${ }^{4}$ The Integrated Graduate Program in Cellular, Molecular, and Biomedical Studies, Columbia University Medical Center, New York, NY

${ }^{5}$ Department of Pathology and Cell Biology, Columbia University Medical Center, New York, NY

${ }^{6}$ Department of Biological Sciences, Columbia University, New York, NY

${ }^{7}$ Taub Institute for Research on Alzheimer's Disease and the Aging Brain, Columbia University Medical Center, New York, NY

${ }^{8}$ UCB Biopharma, Braine l'Alleud, Belgium

${ }^{9}$ Molecular and Cell Biology Laboratory- Salk Institute for Biological Studies, La Jolla, CA 92037, USA

\# These authors contributed equally to this work.

* Address correspondence to:

Franck Polleux, Ph.D.

Columbia University

Department of Neuroscience

Mortimer B. Zuckerman Mind Brain Behavior Institute

Kavli Institute for Brain Science

Jerome L. Greene Science Center

3227 Broadway

L-5-050, MC 9853

New York, NY 10027

fp2304@columbia.edu

Tel: 212-853-0407 


\section{Abstract}

During the early stages of Alzheimer's disease (AD) in both mouse models and human patients, soluble forms of Amyloid- $\beta$ 1-42 oligomers (A 3420$)$ trigger loss of excitatory synapses (synaptotoxicity) in cortical and hippocampal pyramidal neurons (PNs) prior to the formation of insoluble $A \beta$ plaques. We observed a spatially restricted structural remodeling of mitochondria in the apical tufts of CA1 PNs dendrites in the hAPPSWE,IND transgenic AD mouse model (J20), corresponding to the dendritic domain receiving presynaptic inputs from the entorhinal cortex and where the earliest synaptic loss is detected in vivo. We also observed significant loss of mitochondrial biomass in human neurons derived from a new model of human ES cells where CRISPR-Cas9-mediated genome engineering was used to introduce the 'Swedish' mutation biallelically (APP ${ }^{S W E / S W E}$ ). Recent work uncovered that $A \beta 420$ mediates synaptic loss by overactivating the CAMKK2-AMPK kinase dyad, and that AMPK is a central regulator of mitochondria homeostasis in non-neuronal cells. Here, we demonstrate that $A \beta 420$-dependent over-activation of CAMKK2-AMPK mediates synaptic loss through coordinated MFF-dependent mitochondrial fission and ULK2-dependent mitophagy in dendrites of PNs. We also found that the ability of A 3420 -dependent mitochondrial remodeling to trigger synaptic loss requires the ability of AMPK to phosphorylate Tau on Serine 262. Our results uncover a unifying stress-response pathway triggered by $A \beta O$ and causally linking structural remodeling of dendritic mitochondria to synaptic loss.

\section{Introduction}

Alzheimer's disease (AD) is the most prevalent form of dementia in humans leading to socially devastating cognitive defects with no effective treatment. AD is characterized by two hallmark lesions including amyloid plaques composed of fibrillar forms of Amyloid- $\beta(A \beta)$ and neurofibrillary tangles (NFTs) composed of aggregated, hyperphosphorylated Tau. Before the formation of amyloid plaques and NFTs, amyloidogenic processing of amyloid precursor protein (APP) by $\beta$ - and $\gamma$-secretase produces abnormal accumulation of a 42-amino acid-long amyloid beta (A 342$)$ peptide. $A \beta 42$ peptides have a strong ability to oligomerize and form dimers, trimers, and higher order oligomers that ultimately fibrillate to form $A \beta$ plaques (Sheng et al., 2012). Oligomeric forms of $A \beta 42$ (Aß420) lead to early loss of excitatory synapses (synaptotoxic effects) in cortical and hippocampal pyramidal neurons (PNs) before plaque formation, strongly suggesting that synaptotoxicity is an early event in the disease progression triggered by soluble Aß42o (Hong et al., 2016; Mairet-Coello et al., 2013; Masliah et al., 2001; Moolman et al., 2004; Shankar et al., 2007). This phenotype has been observed in various AD mouse models including the J20 transgenic mouse model (APPSWE, IND) where progressive loss of excitatory synaptic connections occur prior to A $\beta$ plaque formation (Jacobsen et al., 2006; Mairet-Coello et al., 2013; Mucke et al., 2000a). Soluble A 420 extracted biochemically from AD patients or produced synthetically also lead to rapid synaptic loss in PNs in vitro (Jin et al., 2011; Lacor et al., 2004; Lacor et al., 2007; Mairet-Coello et al., 2013; Shankar et al., 2007; Shankar et al., 2008).

A second phenotype observed in the early stages of $A D$ progression is structural abnormalities of mitochondria. Because of the terminal postmitotic status and extreme degree of compartmentalization of neurons of the central nervous system (CNS), maintaining mitochondrial homeostasis in neurons is highly dependent on the dynamic balance of fission/fusion and efficient autophagic degradation of aged and damaged mitochondria (Ashrafi and Schwarz, 2015; Lewis et al., 2018; Schwarz, 2013). Mitochondrial fission is carried out by a GTPase protein called Drp1 that needs to be recruited to the outer mitochondrial membrane (OMM) by various proteins including MFF, Fis1, MiD49, and MiD51. The fusion machinery includes Mfn1/2 that is involved in fusion of OMM and OPA1 involved in inner mitochondrial membrane fusion (Burte et al., 2015; Chan, 2006; Youle and van der Bliek, 2012). PNs show strikingly 
compartmentalized morphology of their mitochondria in the somatodendritic versus axonal domains (Lewis et al., 2018). In most cells, mitochondria play critical physiological functions that might differ between dendrites and axons such as ATP production, $\mathrm{Ca}^{2+}$ buffering, and/or lipid biosynthesis (Lewis et al., 2013). Recent evidence points to significant structural remodeling of dendritic mitochondria in hippocampal neurons in multiple AD mouse models and AD patients (Burte et al., 2015; Du et al., 2010; Itoh et al., 2013; Wang et al., 2009a; Xie et al., 2013; Zhang et al., 2016), which is one of the earliest affected brain regions in AD in mouse models and AD patients (Arriagada et al., 1992; Busche et al., 2012; David et al., 2005; Eckert et al., 2008; Rhein et al., 2009; Serrano-Pozo et al., 2011). However, the role played by changes in mitochondria structure and/or function in the disease progression in vivo, especially in relation to synaptic loss, remains untested. Moreover, the molecular mechanisms causally linking changes in mitochondria structure/function and synaptic loss remain unknown in the context of AD pathophysiology.

Results from our lab as well as others demonstrated that in neurons, A 3420 over-activates AMP-activated kinase (AMPK) in a Calcium/calmodulin Kinase Kinase protein 2 (CAMKK2)-dependent manner and preventing CAMKK2 or AMPK over-activation either pharmacologically or genetically protects hippocampal neurons from A 3420 -dependent synaptic loss in vitro and in the hAPP ${ }^{S W E}$, IND transgenic mouse model (J20) in vivo (Ma et al., 2014; Mairet-Coello et al., 2013; Son et al., 2012; Thornton et al., 2011). Importantly, AMPK is over-activated in the brain of AD patients where catalytically active AMPK accumulates in pyramidal neurons of the cortex and hippocampus (Fang et al., 2019; Vingtdeux et al., 2011). In non-neuronal cells, AMPK is a key metabolic sensor that is catalytically activated upon increasing levels of AMP/ADP (when ATP levels drop) and regulates various downstream effectors involved in maintaining mitochondrial integrity in part via two recently identified downstream effectors: mitochondrial fission factor (MFF) and Unc-51 like autophagy activating kinases 1/2 (ULK1/2) (Egan et al., 2011; Toyama et al., 2016). MFF is an outer mitochondrial protein with two identified AMPK phosphorylation sites. Upon AMPK activation, MFF enhances the recruitment of the fission protein Drp1, which is important for efficient fragmentation of mitochondria (Toyama et al., 2016), a step thought to be required for efficient mitophagy. The second and coordinated step regulated by AMPK activation are the ULK proteins. ULK1 (also called Atg1) has multiple AMPK phosphorylation sites and functions in an evolutionarily conserved process called autophagy. Autophagy is involved in degrading cytoplasmic components via lysosomes, often under nutrient stress to obtain energy. Importantly, autophagy is the only known mechanism for degrading organelles including mitochondria (mitophagy). Mounting evidence has also implicated altered autophagy in the pathogenesis of AD as abnormal autophagic vacuole (AV) build-up in neurites of AD patients at least at late stages of the disease progression (Nixon, 2013). Therefore, we wanted to test if the CAMKK2-AMPK pathway could be the first pathway identified to coherently regulate the major $A D$ phenotypes and more precisely if $A \beta 420$-dependent over-activation of AMPK triggers synaptic loss through its ability to mediate mitochondrial structural remodeling.

In the present study, we first reveal that hippocampal CA1 PNs display remarkably compartmentalized structural remodeling of dendritic mitochondria in the J20 AD mouse model in vivo: we observe significant loss of mitochondrial biomass only in the apical tuft dendrites of CA1 PNs which, most significantly, corresponds to the dendritic compartment in the hippocampus where loss excitatory synapses can be first observed in this and other AD mouse models (Siskova et al., 2014). We demonstrate that $A \beta 420$ application induces sequential mitochondrial fission and loss of mitochondrial biomass through mitophagy in dendrites of cortical PNs via coordinated phosphorylation and activation of two critical AMPK effectors: MFF and ULK2. Importantly, individual manipulation of either MFF or ULK2 prevents Aß42o-dependent dendritic spine loss, demonstrating a causal link between A 3420 -dependent mitochondria structural remodeling and synaptic maintenance. Finally, we previously identified that A $3420-$ dependent AMPK phosphorylates Tau at Serine 262 and that preventing S262 phosphorylation by AMPK protected cortical 
PNs from A 4420 -induced synaptic loss. Interestingly, we show that that expression of Tau-S262A is protective towards $A \beta 420$-induced dendritic mitochondrial remodeling and synaptic loss in cortical PNs. Our results (1) demonstrate that the CAMKK2-AMPK stress-response pathway mediates A $3420-$ dependent synaptic loss through Tau-S262 and MFF-ULK2-dependent remodeling of dendritic mitochondria, (2) provide the first 'unifying' stress response pathway triggering many of the cellular defects reported in AD mouse models and in the brain of AD patients during early stages of the disease progression, such as AB42o-dependent Tau phosphorylation, defect in $\mathrm{Ca}^{2+}$ homeostasis, increased autophagy and defective mitochondrial integrity (reviewed in (Sheng et al., 2012)) and (3) provide the first causal link between $A \beta$ - and Tau-dependent dendritic mitochondrial remodeling and synaptic loss.

\section{Results}

\section{Spatially restricted structural remodeling of mitochondria and synaptic loss in apical tufts dendrites of CA1 hippocampal PNs in the APP ${ }^{\text {SWE, IND }}$ mouse model}

The J20 mouse line is a powerful model to study the effect of amyloidosis in AD pathogenesis as it expresses the human Amyloid Precursor Protein (APP) carrying two mutations found in familial forms of AD (APP SWE, IND i.e. KM670.NL and V717F called J20 model thereafter) and show A 420 accumulation in the hippocampus (Mucke et al., 2000a). For this study, we chose to focus on the analysis of mitochondrial morphology and dendritic spine density in CA1 hippocampal neurons, which are among the first and the most affected in AD patients and AD mouse models (Perez-Cruz et al., 2011; Pozueta et al., 2013; Serrano-Pozo et al., 2011; Siskova et al., 2014). Using sparse in utero hippocampal or cortical electroporation $(\mathrm{E} 15 \rightarrow \mathrm{P} 21)$, we were able to visualize dendritic mitochondria morphology and spine density in optically-isolated dendritic segments of hippocampal CA1 PNs or layer 2/3 pyramidal neurons in vivo (Fig. S1). Interestingly, in WT mice, layer $2 / 3$ cortical PNs display elongated and fused dendritic mitochondria, forming a complex network throughout the entire dendritic arbor (Fig. S1B, D, F, H) (Lewis et al., 2018). In sharp contrast, CA1 PNs display a high degree of compartmentalization of mitochondrial morphology in dendrites of WT mice: in basal and apical oblique dendrites, mitochondria are mostly small and punctate whereas the distal apical dendrites contain elongated and fused mitochondria ((Fig. S1A, C, E, G). The transition between these two types of mitochondrial morphology is sharp and corresponds to the boundary between the hippocampal layer stratum radiatum (SR; receiving primarily inputs from CA3 PNs; Fig. 3B) and stratum lacunosum-moleculare (SLM; receiving exclusively presynaptic inputs from the medial entorhinal cortex MEC; Fig. 3B).

We applied the same experimental approach in both WT and J20 littermate mice and examined mitochondrial morphology and spine density in 3-month-old mice, a time-point where abundant $A \beta 420$ can be detected but preceding appearance of amyloid plaques (Mucke et al., 2000a). This analysis revealed that, consistent with what we observed in hippocampus of WT mice at P21 (Fig. S1), 3 monthold WT and J20 mice both display mostly small, punctate mitochondria in basal and apical oblique dendrites (Fig. 1A). This is accompanied by a trend but not significant decrease in spine density in basal and apical oblique dendrites (Fig. 1A,C). However, the distal apical dendrites of the J20 mice showed a dramatic and significant reduction of both mitochondria length and density/biomass as well as a significant decreased in spine density compared to WT littermates (Fig. 1A, C, E). These results reveal (1) a striking and unique degree of compartmentalization of mitochondrial morphology in dendrites of CA1 PNs not observed in cortical layer 2/3 PNs and (2) a spatially restricted loss of mitochondrial biomass and spine density specifically in the apical tuft dendrites of CA1 PNs in the J20 mouse model. This cooccurrence of mitochondrial remodeling and decreased spine density raised the possibility, tested below, of a potential causal relationship between A 3420 -dependent mitochondrial remodeling and synaptic maintenance. 


\section{$A \beta 420$ induces synaptic loss and dendritic mitochondrial remodeling in the same time frame}

In order to examine the temporal relationship between mitochondrial remodeling and decreased spine density, we wanted to adopt an in vitro model that would allow us to control the timing of $A \beta 420$ application. We turned to long-term cortical PNs cultures (Mairet-Coello et al., 2013) which are synaptically connected and display uniformly fused mitochondria throughout their dendritic arbor in vitro (Fig. 2) as observed in vivo (Fig. 1 and S1). We observed significant, time-dependent changes in dendritic mitochondrial structure following acute $A \beta 420$ application including both a reduction of their length (potentially through increased fission) and reduced mitochondrial density/biomass (Fig. 2A'-C'; Fig. 2D-E). In the same neurons, we observed a progressive reduction in dendritic spine density in $A \beta 420$ treated neurons between 14 and 24hr following A 3420 application (Fig. 2A-C, Fig. 2F). No significant changes in mitochondria morphology were observed in the soma or the axon of the same neurons (data not shown) or, as previously reported, when control inverted peptide (INV42) were applied at similar concentrations than Aß42o (300-450nM; Fig. S2E) (Lacor et al., 2004; Lacor et al., 2007; Mairet-Coello et al., 2013; Shankar et al., 2007). We previously published that at this dose and duration, A 3420 application does not affect neuronal viability, strongly suggesting that the effects we observe are not a secondary consequence of compromising neuronal survival (Mairet-Coello et al., 2013). Taken together, our results highlight that $A \beta 420$ application triggers a significant degree of time-dependent, structural remodeling of dendritic mitochondria including reduction of mitochondrial biomass concomitant with a reduction in spine density, which is a reliable index of excitatory synapse number in dendrites of PNs (Harnett et al., 2012).

These results show that in vivo exposure of CA1 hippocampal PNs to $A \beta 420$ resulting from overexpression of APP ${ }^{S W E, I N D}$ in the J20 AD mouse model or direct application of synthetic A 3420 at 300-450 $\mathrm{nM}$ for $24 \mathrm{~h}$ in mature cortical PNs in vitro leads to striking loss of dendritic mitochondrial biomass and synaptotoxicity. However, two potential criticisms of these approaches are (1) that they both use mouse models which could differ from the way human neurons could respond and (2) that both approaches rely on exposure of cortical or hippocampal PNs to high levels of A 3420 . In order to address both points, we generated a novel human embryonic stem cell (hESC) APPSWE/SWE knockin model. CRISPR-Cas9mediated genome engineering was used to introduce to introduce the APP ${ }^{S W E}$ mutation into both alleles of the H9 hESC cell line (WA09, WiCell). Previous knockin of the Swedish mutation into a human PSC line demonstrated increased endogenous APP processing and generation of A $\beta 420$ in an allelic dosedependent manner (Paquet et al., 2016).

APP ${ }^{S W E}$ knockin hESCs and their isogenic H9 parent control line were transdifferentiated into glutamatergic cortical neurons (iNs) using doxycycline-inducible Neurogenin2 (Ngn2) expression (Zhang et al., 2013). Following efficient Ngn2-P2A-eGFP-induced neuronal differentiation (see Methods), we transiently transfected these neurons in a sparse manner with a mitochondrial fluorescent reporter (mitoDsRed) and quantified dendritic mitochondrial morphology in control H9 and isogenic APPSWE/SWE. derived neurons (Fig. S3A). Our results demonstrate a significant reduction in dendritic mitochondrial density (Fig. S3B) and dendritic mitochondrial length (Fig. S2C) in APP ${ }^{S W E / S W E}$ human neurons compared to isogenic control neurons. As previously observed in cortical and hippocampal neurons in vitro and in vivo (Mairet-Coello et al., 2013), we also found increased AMPK activation in APPSWE/SWE neurons compared to isogenic control neurons, as measured biochemically by the ratio of phosphorylated AMPKT172 to total AMPK (Fig. S3D-E). These results demonstrate that endogenous levels of Amyloid- $\beta$ generated by processing of APP in human mutant neurons can trigger dendritic mitochondrial remodeling and loss of biomass as well as AMPK over-activation.

A 3420 application induces local mitophagy in dendrites 
We next tested whether changes in mitochondrial biomass observed upon $A \beta 420$ treatment is triggered in dendrites of PNs by local mitophagy. To that end, we examined the dynamics of autophagosomes, lysosomes, autolysosomes as well as changes in mitochondrial biomass in cortical PNs upon $A \beta 420$ treatment using sparse ex utero electroporation of plasmids encoding mito-mTagBFP2, LAMP1mEmerald, and RFP-LC3 to visualize mitochondria, lysosomes, and autophagosomes, respectively. Cortical PNs were cultured for at least 21DIV in high-density cultures, where they display mature synapses (Mairet-Coello et al., 2013), and imaged live using time-lapse confocal microscopy every 15 minutes for 14 hours following treatment with either control (vehicle) or Aß42o (Fig. 3). In control treated neurons, we observed little to no change in mitochondrial biomass or in the dynamics of LC3+ (autophagosomes), LAMP1+ (lysosomes) or LC3+/LAMP1+ (autolysosomes) vesicles (Fig. 3A-A"). On the contrary, upon A 3420 treatment, we observed a significant accumulation of both LC3+ and LAMP1+ puncta in dendrites over time as visualized in Fig. 3A (see kymograph in Fig. 3A"). In dendrites exposed to $A \beta 420$, many of the LC3+ puncta became co-localized with LAMP1+, suggesting these autolysosomes often occur locally. LC3+/LAMP1+ autolysosomes do form occasionally in control dendrites (Fig. 3A"'B). As shown in Fig. 3B, treatment with $A \beta 420$ leads to an almost 5-fold increase in the percentage of dendritic segments with increased LC3+/LAMP1+ autolysosomes accumulation compared to control treatment. Both LC3 and LAMP1 normalized fluorescence intensity showed time-dependent increases upon $A \beta 420$ application, highlighting local biogenesis of autophagic vacuoles and their coalescence with lysosomes in dendritic shafts upon $A \beta 420$ treatment, respectively (Fig. 2C-E). We also observed a significant reduction in mitochondria fluorescence (as measured via mt-mTAGBFP2, (Fig. 2E)) in a spatially restricted area close to LC3+/LAMP1+ puncta as indicated by the yellow arrow in the representative image (Fig. 3A) and the kymograph (Fig. 3A"). Not all LC3+/LAMP1+ puncta correlated with loss of a mitochondria within $14 \mathrm{~h}$ of time-lapse imaging as indicated by the red arrow in the representative image (Fig. 3A-A"). To quantify spatial dynamics of mitophagy, we defined a "mitophagy index' along segments of dendrites by measuring the intensity of mito-mTagBFP2 fluorescence using line scan intensity measurements in regions of interest (ROI) with or without LC3+/LAMP1+ puncta. This index reflects the probability of loss of mitochondrial biomass in or out of areas where autophagosomes and lysosomes coalesce to generate autolysosomes. In control conditions, the formation of LC3+/LAMP1+ puncta is not only a rare event (Fig. 3A-B), but the presence of LC3+/LAMP1+ puncta do not result in loss of mitochondria biomass therefore suggesting that basal levels of mitophagy are exceedingly low in dendrites of cortical PNs in these conditions (Fig. 3B-C). In contrast, upon A 4420 treatment, we frequently observed spatially restricted mitophagy events, only found in ROls of the dendrites that display LC3+/LAMP1+ autolysosomes demonstrating that significant levels of mitophagy occur locally in dendrites of cortical PNs upon Aß42o treatment (Fig. 3B-C).

\section{Aß420-dependent mitochondrial fragmentation and synaptotoxicity are mediated by CAMKK2- AMPK over-activation}

We have previously shown that Aß42o-dependent over-activation of the CAMKK2-AMPK kinase dyad mediates excitatory synaptic loss both in primary cortical PNs culture and in hippocampal PNs in the J20 AD mouse model, (Mairet-Coello et al., 2013). Since AMPK is a central regulator of mitochondrial homeostasis (Garcia and Shaw, 2017; Herzig and Shaw, 2018), we wanted to test if the CAMKK2-AMPK pathway was required for $A \beta 420$-induced dendritic mitochondrial remodeling in primary cortical PNs cultures in vitro using a conditional double floxed mouse line (AMPKa1 $1^{\mathrm{F} / \mathrm{F}} / \alpha 2^{\mathrm{F} / \mathrm{F}}$ ). The CA1 hippocampal region of $A M P K \alpha 1^{\mathrm{F} / \mathrm{F}} / \alpha 2^{\mathrm{F} / \mathrm{F}}$ embryos was electroporated at E15.5 with (1) a control plasmid lacking Cre recombinase or a plasmid expressing Cre recombinase along (2) with Venus fluorescent protein to visualize spine morphology and (3) mt-dsRed to examine mitochondrial morphology. Quantitative analysis indicated that in basal conditions AMPKa1/a2 null neurons have spine and mitochondria 
densities indistinguishable from wildtype neurons. Strikingly, AMPKa1/a2 null neurons are completely protected from A 3420 -induced mitochondrial density reduction (Fig. 4A) as well as synaptotoxicity as previously reported (Mairet-Coello et al., 2013). This demonstrates that over-activation of AMPK is required for both $A \beta 420$-induced mitochondria structural remodeling and spine loss in dendrites of PNs. AMPK has two upstream regulatory kinases namely, LKB1 and CAMKK2 which phosphorylates AMPK $\alpha$ subunit on T172 and significantly increases its catalytic activity (Garcia and Shaw, 2017). However, in neurons, CAMKK2 has been identified as the primary upstream regulator of AMPK (Hardie et al., 2012; Mairet-Coello et al., 2013; Son et al., 2012). Therefore, we assessed if pharmacologically blocking CAMKK2 acutely interfered with $A \beta 420$ effects on mitochondrial structural remodeling and dendritic spine loss. Pretreatment of primary neuronal cultures with STO-609, a specific CAMKK2 inhibitor (Tokumitsu et al., 2002), completely blocked Aß42o-induced mitochondrial structural remodeling (Fig. S4A-C) as well as synaptotoxicity (Fig S4A; S4D) as previously published (Mairet-Coello et al., 2013).

Aß420-induced MFF over-activation causally links mitochondrial fragmentation and synaptic loss In non-neuronal cells, AMPK has been identified as a major metabolic sensor regulating ATP homeostasis upon induction of metabolic stress through its ability to phosphorylate many downstream effectors involved in various aspects of metabolic homeostasis (Hardie et al., 2012; Herzig and Shaw, 2018). As part of this metabolic stress response, AMPK regulates mitochondrial fission through direct phosphorylation of mitochondrial fission factor (MFF) which enhances its ability to recruit Drp1 to the outer mitochondria and promotes mitochondrial fission (Toyama et al., 2016). To that end, we tested if MFF is the effector mediating AMPK-dependent A 420 -induced mitochondrial fission and if blocking MFF-dependent fission can prevent synaptic loss. Upon exposure of $A \beta 420$, cortical PNs in vitro that express MFF shRNA (knockdown efficacy shown in Lewis et al., 2018) are protected from loss of mitochondrial biomass in dendrites (Fig. 5A-C) and from synaptic loss (Fig. 5A and 5D). Interestingly, knockdown of MFF in basal condition does not affect either the dendritic mitochondrial length and density or dendritic spine density, strongly suggesting that the signaling pathway we have identified is a bona fide stress response pathway in dendrites upon $A \beta 420$ exposure, as we have previously hypothesized (Mairet-Coello and Polleux, 2014). Altogether, these results suggest a causal relationship between Aß42o-induced MFF-dependent dendritic mitochondria fission and dendritic spine loss.

\section{Aß420-induced MFF phosphorylation by AMPK is required for dendritic mitochondrial fragmentation and synaptic loss.}

To determine if MFF is phosphorylated by A 4 420-dependent AMPK over-activation in neurons, we measured AMPK-mediated phosphorylation on MFF at Serine 146 (S146; one of the AMPK phosphorylation site of Isoform 3 enriched in the brain; (Ducommun et al., 2015)) and total MFF in primary cortical neurons (Fig. S5). As a positive control to show that the MFF S146 site is regulated by AMPK activation, we over-expressed the human isoform of MFF in Neuro2A (N2A) neuroblastoma cells and treated with or without Metformin, a potent activator of AMPK (Toyama et al., 2016) (Fig. S5). Firstly, Aß42o application for $24 \mathrm{~h}$ on long-term cortical PNs cultures showed a significant increase in AMPK phosphorylation at T172 site as previously reported (Mairet-Coello et al., 2013; Thornton et al., 2011) (Fig. S5). As a consequence of AMPK activation, we observe an increase in phosphorylation of MFF at S146 in cortical neurons upon A 3420 application which is comparable to Metformin treatment of N2A cells (Fig. S5). This experiment demonstrates that $A \beta 420$ leads to AMPK dependent phosphorylation and activation of MFF in cortical PNs.

In order to test if AMPK-dependent phosphorylation of MFF is required for Aß42o-dependent mitochondrial remodeling, we took a gene replacement approach where we identified low expression levels of wild-type human MFF ( $h M F F$ WT not targeted by shRNA directed against mouse MFF) and 
hMFF carrying two point mutations (S155A and S172A referred to as $h M F F$ AA previously shown to abolish AMPK-mediated MFF function in mitochondrial fission; (Toyama et al., 2016)) that, when combined with mouse MFF shRNA, does not lead to mitochondrial fragmentation in control conditions i.e. does not lead to MF over-expression which was previously shown to be sufficient to induce dendritic mitochondrial fragmentation (Toyama et al., 2016). Primary cortical PNs expressing these titrated levels of hMFF WT showed both mitochondrial and spine loss following $24 \mathrm{hr}$ of A 3420 treatment (Fig. 6C-F) to levels comparable to control neurons exposed to $A \beta 420$, whereas neurons expressing titrated levels of hMFF AA were protected from A 420 -induced mitochondrial structural remodeling and spine loss (Fig. 6C-F). These results demonstrate a causal role of $A \beta 420$-triggered mitochondrial remodeling induced by AMPK-mediated phosphorylation of MFF and synaptic loss.

\section{Activation of ULK2 by AMPK leads to loss of mitochondrial biomass following MFF-dependent mitochondrial fission}

In non-neuronal cells under conditions of metabolic stress, AMPK activation not only triggers increased mitochondrial fission through phosphorylation and activation but also trigger mitophagy by directly phosphorylating the autophagy initiating kinase, ULK1 (Atg1). Since we observed (1) increased mitophagy in dendrites exposed to $A \beta 420$ and (2) a significant decrease in mitochondrial biomass following increased AMPK-MFF-dependent fission between 14-24h of A 3420 treatment (Fig. 1 and 5), we hypothesized that over-activation of AMPK by A 3420 also activates the observed increased levels of mitophagy by activating ULK1 and/or ULK2, two isoforms of ULK implicated in mitophagy and highly expressed in cortical neurons (Egan et al., 2011; Kim et al., 2011).

We tested first if either one or both of the two isoforms of the ULK1/2 mediate Aß42o-induced synaptotoxicity by performing shRNA-mediated knockdown of either ULK1 or ULK2 (Fig. S6A). Interestingly, we found that knockdown ULK2, but not ULK1, protected cortical PNs from Aß42o-induced spine loss at $24 \mathrm{~h}$ (Fig. S6B-C). We then tested if loss of ULK2 could protect A 420 o-treated neurons from loss of mitochondrial biomass. We sparsely expressed Cre-recombinase in primary cortical PNs isolated from a conditional ULK2 mouse line (ULK2 ${ }^{\mathrm{F} / F}$ ) (Cheong et al., 2011) along with Venus as a cell filler and mt-dsRed to visualize mitochondria. Loss of ULK2 completely blocks Aß42o-induced loss of mitochondrial biomass, however, interestingly, dendritic mitochondria remain fragmented compared to the controls (Fig. 7A-C), compatible with the AMPK-MFF dependent induction of mitochondrial fission being intact in these ULK2-deficient neurons. This further supports the current idea that mitochondrial fission and mitophagy are coupled events such that mitochondrial fragmentation must precede mitophagy (Chan, 2006; Schwarz, 2013). Interestingly, preservation of mitochondrial density/biomass upon ULK2 conditional deletion was sufficient to rescue A 4420 -induced synaptotoxicity (Fig.7A and D). Therefore, our data suggest that these two parallel effectors (MFF and ULK2) coincidentally activated by AMPK act in a concerted manner to couple fission and mitophagy in neuronal dendrites and most importantly that loss of mitochondrial biomass triggered by $A \beta 420$ is causally linked to synaptic loss.

\section{AMPK phosphorylation of ULK2 is required for A 3420 -induced dendritic mitophagy and synaptic loss}

We next tested if AMPK-mediated phosphorylation of ULK2 is required for Aß42o-dependent synaptotoxic effects in cortical PNs. Extensive studies in non-neuronal cells have established that the closest ortholog of ULK2, ULK1, is phosphorylated and thereby catalytically activated by AMPK and plays a key role in regulating mitophagy (Egan et al., 2011; Kim et al., 2011). Although the two orthologs seem to have redundant functions in non-neuronal cells, the above results uncovered that, in cortical PNs that only downregulation of ULK2, but not ULK1, completely blocks A 342 -mediated synaptotoxicity (Fig. S6). Therefore, we performed a sequence homology analysis of candidate AMPK sites identified in ULK1 and 
compared them to ULK2. Two independent groups collectively reported six AMPK-mediated phosphorylation sites on ULK1 (Egan et al., 2011; Kim et al., 2011). Using primary sequence alignment between human and mouse ULK1/2, we have found that four of these ULK1 phosphorylation sites are conserved in ULK2 and are predicted as bona fide AMPK consensus phosphorylation sites (Fig.8A and Fig. S7; S309/T441/S528/S547). To verify that these sites are the four most prevalent AMPK target sites in ULK2, we mutated these four candidate $S / T$ residues to alanine in ULK2 (ULK2-4SA) and overexpressed them in HEK293T cells. We then performed immunoprecipitation (IP) of Flag-tagged ULK2 WT and 4SA mutant from cells that are either treated with DMSO or AMPK direct agonist 991. Overexpression of constitutively active AMPK (CA-AMPK) in the presence of ULK2 WT or ULK2 4SA mutant conditions were used as a positive control. Immunoprecipitated Flag-ULK2 WT or Flag-ULK2 4SA were then immunoblotted with an antibody recognizing the optimal AMPK phospho-substrate motif. We observed basal levels of phosphorylation of ULK2 WT in untreated control which increases upon AMPK activation or when co-expressed with CA-AMPK (Fig. 8B). Importantly, ULK2 phosphorylation was completely abolished in the 4SA mutant (Fig. 8B), strongly suggesting that these four S/T sites are the main AMPK phosphorylation sites in ULK2.

Finally, we tested if ULK2 phosphorylation by AMPK is required for the loss of mitochondrial biomass and the synaptic loss induced $A \beta 420$ application. We performed gene replacement experiments taking advantage of ULK2 ${ }^{\mathrm{F} / \mathrm{F}}$ primary cortical PNs co-electroporated with Cre (or delta Cre as negative control) along with ULK2-WT (positive control) or ULK2-4SA or kinase inactive ULK2 (ULK2-KI as negative control). We found that expression of ULK2-WT in ULK2-null cortical PNs enabled both loss of mitochondrial biomass and synaptic loss induced by Aß42o, but the re-introduction of ULK2 KI or ULK2 4SA had no effect on mitochondrial biomass and did not prevent dendritic spine loss (Fig. 8C-F). These results confirm that AMPK mediated phosphorylation and activation of ULK2 mediate Aß42o-dependent synaptic loss through its ability to induce mitophagy and loss of mitochondrial biomass.

\section{Phosphorylation of Tau on Serine 262 is required for A $\beta 420$-induced mitochondrial remodeling and synaptotoxicity}

We and others previously shown that AMPK phosphorylates human Tau (hTau) on the KGxS motif present at Serine 262 embedded within the R1 microtubule binding domain (Mairet-Coello and Polleux, 2014; Thornton et al., 2011) and that expressing a form of hTau that cannot be phosphorylated at S262 (hTau-S262A) blocked Aß42o-induced synaptic loss in cortical and hippocampal PNs (Mairet-Coello and Polleux, 2014). Since hTau phosphorylation has been involved in Drp1-mediated mitochondrial fission in multiple fly and mouse AD models (DuBoff et al., 2012; Manczak and Reddy, 2012), we tested specifically if AMPK-dependent hTauS262 phosphorylation could link Aß420-induced mitochondrial remodeling and synaptotoxicity. Indeed, expression of hTau-S262A (but not wildtype hTau) protected neurons from Aß420-induced mitochondrial remodeling and synaptotoxicity (Fig. 9A-D). This result strongly suggests that AMPK-dependent Tau phosphorylation on S262 participates to the MFF-ULK2-dependent mitochondrial remodeling and synaptic loss triggered by $A \beta 420$.

\section{Discussion}

Loss of excitatory glutamatergic synapses in cortical and hippocampal PNs has been reported as an early event in AD progression, primarily driven by soluble $A \beta 420$ and Tau hyperphosphorylation (Hsieh et al., 2006; Ittner et al., 2010; Lacor et al., 2007; Mucke et al., 2000a; Mucke et al., 2000b; Roberson et al., 2011; Roberson et al., 2007; Shankar et al., 2007; Sheng et al., 2012). This loss of connectivity is thought to be responsible for the early cognitive defects characterizing AD patients including defective learning and memory (Sheng et al., 2012; Terry et al., 1991). Recently, mitochondrial remodeling and dysfunction in PNs have emerged as a prominent cellular phenotype in AD (Cho et al., 2009; Fang et al., 
2019; Wang et al., 2009a; Wang et al., 2009b; Zhang et al., 2016). However, whether this structural remodeling of dendritic mitochondria is causally linked to excitatory synaptic loss was unknown, in part because the molecular mechanism mediating Aß42o-dependent mitochondrial remodeling are currently unknown.

AMPK is best-characterized as a metabolic sensor in non-neuronal cells (Herzig and Shaw, 2018). Importantly, in AD patients, catalytically active AMPK is abnormally accumulated in the cytoplasm of PNs of CA1, entorhinal cortex, and neocortex (Vingtdeux et al., 2011). AMPK over-activation has been consistently reported in AD patient brain samples (Ma et al., 2014; Vingtdeux et al., 2011), in various AD mouse models (Ma et al., 2014; Mairet-Coello et al., 2013; Son et al., 2012), as well as in vitro upon acute treatment of Aß42o (Ma et al., 2014; Mairet-Coello et al., 2013; Thornton et al., 2011; Yoon et al., 2012). The CAMKK2-AMPK signaling pathway is unique in its involvement in multiple phenotypes observed during the progression of $A D$. The present study and previous reports collectively implicate this signaling pathway as the first to unify multiple phenotypes reported in the brain of $A D$ patients and in $A D$ mouse models, including disruption of $\mathrm{Ca}^{2+}$ homeostasis, APP processing, hTau phosphorylation, mitochondrial remodeling, increased autophagy, and synaptic loss (Marinangeli et al., 2018; Nixon, 2005, 2013; Sheng et al., 2012). There is growing evidence that one of the earliest event upon $A \beta 420$ application is disrupted cytoplasmic $\mathrm{Ca}^{2+}$ homeostasis that drives PNs hyperexcitability (see below) (Arbel-Ornath et al., 2017; Busche et al., 2012; Grienberger et al., 2012; Kuchibhotla et al., 2008; Lerdkrai et al., 2018; Palop and Mucke, 2010). This increased $\mathrm{Ca}^{2+}$ accumulation precedes spine morphological changes (Arbel-Ornath et al., 2017), strongly suggesting that $\mathrm{Ca}^{2+}$-dependent signaling pathways, including the CAMKK2-AMPK pathway, occur early in the disease progression and plays a critical role in reduction of synaptic plasticity and synaptic maintenance (Hsieh et al., 2006; Ma et al., 2014; Malinow, 2012) . Interestingly, Aß42oinduced AMPK over-activation has been found to induce a vicious cycle of increased $A \beta 420$ production via activation of JNK3-dependent APP processing (Yoon et al., 2012). Furthermore, AMPK links the two key players of AD: A 420 and hTau. Catalytically active AMPK has been shown to co-localize with phosphorylated-S262 hTau in brains of AD patients (Vingtdeux et al., 2011), and previous work highlights that hyperactivation of AMPK by $A \beta 420$ and /or other AMPK-like kinases such as NUAK1 leads to Tau phosphorylation on two of its serine residues (S262 and S356; (Lasagna-Reeves et al., 2016; MairetCoello et al., 2013)) embeded in the R1 and R4 microtubule binding domains respectively (Buee et al., 2000). These results identified for the first time the CAMKK2-AMPK signaling pathway as a critical link between Aß42o to Tau phosphorylation in the induction of synaptic loss (Mairet-Coello et al., 2013; Mairet-Coello and Polleux, 2014; Thornton et al., 2011).

In this paper, we identified two new critical effectors of AMPK mediating the effects of A 420 on synaptic loss. We show that AMPK over-activation by $A \beta 420$ coordinates multiple aspects of mitochondrial remodeling by co-regulating mitochondrial fission and mitophagy through its activation of MFF and ULK2, respectively. For the first time, we demonstrate that $A \beta 420$ mediates loss of excitatory synapses through MFF-ULK2-dependent mitochondrial fission and mitophagy. We have identified that Aß42o-mediated AMPK over-activation also signal through phosphorylation of Tau on S262 in parallel to MFF-ULK2 phosphorylation/activation and lead to dendritic mitochondrial remodeling and synapse loss. Further work will be required to understand if A 3420 -induced AMPK-Tau signaling plays a direct role in mitochondrial fission and/or mitophagy machinery or plays an indirect role through its postsynaptic signaling function (Ittner and Gotz, 2011; Ittner et al., 2010) and/or its ability to regulate microtubule dynamics (Qu et al., 2017) thereby indirectly influence mitochondrial remodeling.

Lastly, what makes this molecular mechanism attractive for development of new therapeutic approaches is that it is a true bonafide stress-response signaling pathway (Mairet-Coello and Polleux, 2014) i.e. these AMPK-dependent pathways are dispensable for normal neuronal development and/or synaptic maintenance in adult cortical and hippocampal neurons (Mairet-Coello et al., 2013; Mairet-Coello and 
Polleux, 2014; Williams et al., 2011). Genetic manipulations of AMPK or its downstream effectors do not have any identified phenotypic effects on neurons under basal conditions, but only upon induction of metabolic stress or A 3420 application in neurons.

Mitochondrial remodeling and dysfunction are converging mechanisms of disease pathogenesis shared by multiple neurodegenerative diseases (ND). This is in part explained by the disruptions of mitochondrial homeostasis, motility, and dynamics observed in various ND as well as genetic evidence suggesting a strong association between genes involved in mitochondrial function and various adult on-set ND syndromes (Itoh et al., 2013; Schon and Przedborski, 2011). In AD specifically, altered mitochondrial dynamics have been implicated by the changes of mitochondrial fission and fusion protein expression levels in AD patient brains (Wang et al., 2009a) and various AD mouse models (Wang et al., 2008), as well as altered posttranslational modification of Drp1 thought to disrupt mitochondrial dynamics (Cho et al., 2009; Wang et al., 2009a). In line with our results, Wang, et al., (2009) also observe that in the presence of $A \beta 420$, dendritic mitochondria decrease in length and density, which is correlated with reduced spine density. Interestingly, a recent study suggested a significant degree of mitochondrial structural remodeling in various $A D$ mouse models and $A D$ patients in the $C A 1$ of the hippocampal region using 3D EM reconstructions (Zhang et al., 2016). Our present study further corroborates these observations and provides a molecular mechanism mediating A 3420 -dependent mitochondrial remodeling and synaptic loss during early stages of $A D$.

A second cellular defect thought to contribute to several neurodegenerative diseases including $A D$ is a disruption of autophagy-lysosomal pathway (Nixon, 2013). Although the cellular and molecular mechanisms of autophagy have been examined using non-neuronal cells (Mizushima, 2007), recent results demonstrated unique, compartment-specific regulation of autophagy in neurons. Local biogenesis of autophagosomes has been observed in distal axons and traffic retrogradely towards the soma in order to fuse with lysosomes, whereas autophagosomes in the soma are more stationary (Maday and Holzbaur, 2016). However, the dynamics of autophagy in dendrites remains largely unexplored. Much of what we know on neuronal autophagy has been primarily focused on axonal and presynaptic autophagy dynamics, where it mediates efficient synaptic transmission by regulating the turnover of synaptic proteins (Uytterhoeven et al., 2015). Interestingly, autophagosome (AP) formation occurs in distal axons and can traffic retrogradely to fuse with lysosomes that are localized closer to the soma (Maday and Holzbaur, 2016). Autophagy has received significant attention in $A D$ research, in part due to the abnormal accumulation of AP and autolysosomes (AL) in dystrophic neurites of AD patients (Nixon, 2005). Moreover, familial mutations and polymorphisms associated with $A D$ link to dysfunction of the autophagic-lysosomal pathway. Mutations of the Presenillin (PS) 1 and 2 proteins have been shown to disrupt lysosomal acidification and autophagy (Lee et al., 2015). AD associated genes such as PICALM, BIN1, RAB11, and VPS34 are involved in various initiation, sorting, and trafficking processes of autophagy (Ando et al., 2016; Miyagawa et al., 2016; Morel et al., 2013; Udayar et al., 2013). However, the majority of the cellular phenotypes involving AP and AL buildup in neurons have been described at late stages of $A D$ progression when neurodegeneration involving actual neuronal cell loss occurs (Nixon, 2005) . More recent results suggested that, in AD mouse models, autophagic flux is disrupted (Son et al., 2012) and expression level of mitophagy genes are elevated during the early stages of AD (Sorrentino et al., 2017). Our results demonstrate that A 420 triggers an AMPK-ULK2 dependent increase in mitophagy resulting in a significant local degradation of dendritic mitochondria and most interestingly, that this cell biological step is causally linked with synaptic loss since we rescue dendritic spine loss induced by $A \beta 420$ in cortical PNs where ULK2 phosphorylation by AMPK is disabled.

Our in vivo analysis also reveals a striking and previously unknown degree of compartmentalization of mitochondria morphology in dendrites of CA1 PNs: in basal and apical oblique dendrites, mitochondria are small and punctate whereas the distal apical dendrites contain elongated and fused mitochondria 
(Fig. S1). The transition between these two types of mitochondrial morphology is sharp and actually corresponds to the transition between two hippocampal layers, stratum radiatum (sr) and stratum lacunosum moleculare (s/m) where the earliest excitatory synaptic loss is observed in this mouse model. This is particularly relevant because the synapse made between medial entorhinal cortical inputs and the apical tufts of CA1 PNs in stratum lacunosum moleculare has been described as one of the central synapse defective in late onset forms of AD (Small et al., 2011). Future investigations will need to determine why this synapse is particularly vulnerable during early stages of AD progression.

As discussed above, disruption of $\mathrm{Ca}^{2+}$ homeostasis has been observed in various $\mathrm{AD}$ mouse models (Busche et al., 2012; Grienberger et al., 2012; Lerdkrai et al., 2018), in wildtype PNs exposed to acute treatment of oligomeric $A \beta 42$ in vivo (Arbel-Ornath et al., 2017), and neuronal culture exposed to $A \beta 420$ in vitro (Demuro et al., 2005; Guo et al., 1999). Recent results suggested that disruption of $\mathrm{Ca}^{2+}$ dynamics is largely due to $A \beta 420$, as immune-depletion of $A \beta 420$ can prevent this phenotype (Arbel-Ornath et al., 2017) and increase the population of hyperactive neurons (Busche et al., 2012; Grienberger et al., 2012). Possible explanations of this increased cytoplasmic $\mathrm{Ca}^{2+}$ accumulation include increased mobilization of $\mathrm{Ca}^{2+}$ from extracellular space through NMDAR and VGCC, increased leakage of $\mathrm{Ca}^{2+}$ from intracellular $\mathrm{Ca}^{2+}$ storing organelles such as the ER, and loss of $\mathrm{Ca}^{2+}$ buffering capacity by the neurons. Currently, there is evidence supporting that $\mathrm{Ca}^{2+}$ is derived from the extracellular influx of $\mathrm{Ca}^{2+}$ via NMDAR (ArbelOrnath et al., 2017; Sheng et al., 2012) as well as the $\mathrm{Ca}^{2+}$ released from the ER via the $\mathrm{IP}_{3} \mathrm{R}$ and RyR (Chan et al., 2000; Stutzmann et al., 2004). Regardless of the $\mathrm{Ca}^{2+}$ source, PNs ultimately experience intracellular $\mathrm{Ca}^{2+}$ increase that can activate $\mathrm{Ca}^{2+}$-dependent signaling pathway, including our CAMKK2AMPK pathway. There may be a complex positive feed-forward loop where A 3420 -dependent, fast NMDAR-dependent $\mathrm{Ca}^{2+}$ influx (Arbel-Ornath et al., 2017) over-activates the CAMKK2-AMPK pathway (Mairet-Coello et al., 2013), resulting in reduction of mitochondrial biomass, i.e. mitochondrial matrix volume, and therefore could lead to reduced $\mathrm{Ca}^{2+}$ buffering capacity, further increasing cytoplasmic $\mathrm{Ca}^{2+}$ accumulation and CAMKK2 over-activation. Future work will need to address if the striking but spatially restricted degree of $A \beta 420$-dependent reduction in dendritic mitochondrial biomass and the reduction in spine density identified in the present study in the apical tuft dendrites of CA1 PNs in the J20 AD mouse model contributes to degradation of the spatial tuning properties of these neurons at early stages of the disease progression.

\section{Acknowledgments}

We thank members of the Polleux lab for feedback and discussion, as well as Karen Duff, Ulrich Hengst, Carol Troy for critically evaluating the manuscript. We thank Benoit Viollet (INSERM, France) for providing the AMPK double conditional mouse lines. We thank Fan Wang (Duke University) for providing ULK1 and ULK2 shRNA constructs. We thank Qiaolian Lu and Miyako Hirabayashi for excellent management of our mouse colony. This work was supported by grants from the NIH (NS089456) (FP), a F31 Award from NIH (AL), a K99 award (NS091526) (TLL), the Henry and Marilyn Taub Foundation (AS), the Ludwig Foundation (FP, AS) and an award from the Fondation Roger De Sproelberch (FP). 


\section{Material and methods}

\section{Animals}

Mice were used according to protocols approved by the Institutional Animal Care and Use Committee (IACUC) at Columbia University and in accordance with National Institutes of Health guidelines. Timepregnant CD1 females were purchased from Charles Rivers. 129/SvJ, C57BI/6J nontransgenic mice and hemizygous transgenic mice from line J20 (hereafter referred as J20) (The Jackson Laboratory stock \#006293) were maintained in a 12-hour light/dark cycle. J20 mice express human APP carrying the Swedish and Indiana mutations under a PDGF $\beta$ promoter (Mucke et al., 2000a; Palop et al., 2007). AMPKa $1^{\mathrm{F} / \mathrm{F}} \alpha 2^{\mathrm{F} / \mathrm{F}}$ double conditional knockout mice were a generous gift from Dr. Benoit Viollet (INSERM, Institut Cochin, Paris-France). Ulk2 ${ }^{\mathrm{F} / \mathrm{F}}$ mice were obtained from The Jackson Laboratory stock \#017977 (Cheong et al., 2011). Timed-pregnant females were obtained by overnight breeding with males of the same strain. Noon the day after the breeding was considered as E0.5.

\section{Synthetic $A \beta 42$ oligomers preparation}

A 342 (rPeptide or Creative Peptide) was processed to generate A $\beta 42$ oligomers as described previously (Mairet-Coello et al., 2013). Briefly, lyophilized Aß42 peptide was dissolved in hexafluoro-2-propanol (HFIP; Sigma-Aldrich) for 2 hours to allow monomerization. HFIP was removed by speed vacuum, and the monomers were stored in $-80^{\circ} \mathrm{C}$. A $\beta 42$ monomers were dissolved in anhydrous dimethyl sulfoxide (DMSO) to make a $5 \mathrm{mM}$ solution, then added to cold phenol red-free F12 medium (Life Technology) to make a $100 \mathrm{uM}$ solution. The solution was incubated at $4^{\circ} \mathrm{C}$ for two days and centrifuged at $12,000 \times \mathrm{g}$ for $10 \mathrm{~min}$ at $4^{\circ} \mathrm{C}$ in order to discard the fibrils. The supernatant containing the $4^{\circ} \mathrm{C}$ oligomers were assayed for protein content using the BCA kit (Thermo Fisher Scientific). For control experiments, vehicle treatment corresponded to the same volume of DMSO and F12 media used for $A \beta 420$ treatment and for Figure S2, a peptide corresponding to the inverted sequence of AB42 (INV42; rPeptide and Creative Peptide) was processed as for $A \beta 42$ oligomerization. For western blotting of $A \beta 42$ oligomers, $16.5 \%$ TrisTricine SDS-PAGE was performed. Synthetic A 442 oligomers were prepared in $2 X$ Tricine sample buffer (BIORAD) without reducing agent and resolved by SDS-PAGE. The separated proteins were transferred onto Immobilin-FL PVDF membrane (EMD Millipore), blocked for 1 hour with Odyssey blocking buffer (LICOR) and probed with 6E10 monoclonal antibody (Covance) overnight at $4^{\circ} \mathrm{C}$. Secondary antibody incubation was performed for 1 hour at room temperature with IRDye 680RD goat anti-mouse secondary (LICOR) and the blot was visualized using the Odyssey Imaging system. To estimate the relative concentration of synthetic A 42 peptide monomer versus oligomers (dimers and trimers), we performed quantitative western blotting (see Fig. S2E) and measured the ratio of optical density measured for the monomeric peptide versus the dimer/trimer bands. Using this approach, we estimated that a $1 \mu \mathrm{M}$ concentration of oligomerized peptide, contains approximately $300-450 \mathrm{nM}$ of effective dimers/trimers.

\section{Primary cortical culture and ex utero electroporation}

Cortices from E15.5 mouse embryos were dissected followed by dissociation in complete Hank's balanced salt solution (cHBSS) containing papain (Worthington) and DNase I (100ug/mL, Sigma) for 15 minutes at $37^{\circ} \mathrm{C}$, washed three times, and manually triturated in DNase I $(100 \mathrm{ug} / \mathrm{mL})$ containing neurobasal medium (Life Technology) supplemented with B27 (1x, Thermo Fischer Scientific,), FBS (2.5\%, Gibson) N2 (1x, Thermo Fischer Scientific), glutaMAX (2mM, Gibco). Cells were plated at $10.0 \mathrm{X}$ $10^{4}$ cells per $35 \mathrm{~mm}$ glass bottom dish (Mattek) that has been coated with poly-D-lysine $(1 \mathrm{mg} / \mathrm{mL}$, Sigma) over night. One-third of the medium was changed every 5 days thereafter with non-FBS containing medium and maintained for $20-25$ days in $5 \% \mathrm{CO}_{2}$ incubator at $37^{\circ} \mathrm{C}$. Ex utero electroporation was performed as previously published (Courchet et al., 2013). See the Supplemental Experimental Procedures for details and constructs. Plasmids used for ex utero electroporation where all in pCAG 
vector backbone (Guerrier et al., 2009) expressing the following cDNAs: LAMP1-mEmerald (Lewis et al., 2016), mito-DsRed and Venus (Courchet et al., 2013), mRFP-LC3 (originally from https://www.addgene.org/21075/ and subcloned into pCAG).

\section{In utero hippocampal electroporation}

In utero electroporation targeting the hippocampus was performed as previously described (Hand and Polleux, 2011; Mairet-Coello et al., 2013) with slight modifications as described in (Szczurkowska et al., 2016) to target the embryonic hippocampus at E15.5. See Supplemental Experimental Procedures for more details.

\section{Imaging}

Imaging on dissociated neurons was performed between 20-25 DIV in 1,024 x 1,024 resolution with a Nikon Ti-E microscope equipped with A1R laser-scanning confocal microscope using the Nikon software NIS-Elements (Nikon, Melville, NY, USA). We used the following objective lenses (Nikon): 10x PlanApo; NA 0.45 (for images of hippocampal slices), 60X Apo TIRF; NA 1.49 (for analysis of spine density and mitochondrial morphology in cultured neurons), 100x H-TIRF; and NA 1.49 (for analysis of spine densities and mitochondrial morphology in brain slices). Dendritic spine density was quantified on secondary or tertiary dendritic branches that were proximal to the cell body, on z projections for cultured neurons and in the depth of the $z$ stack for slices, using FiJi software (ImageJ; NIH). See Supplemental Experimental Procedures for more details.

\section{hESC culture}

H9 (WA09; WiCell) and its genome edited derivatives were maintained on StemFlex (Life) and Cultrex substrate (Biotechne), and routinely split $\sim$ twice a week with ReLeSR (Stem Cell Technologies) in the presence of ROCKi (Y-27632; Selleckchem). H9 is a commercially available hESC line on the NIH Registry (\# 0062) and Dr. Sproul has approval to genome edit and differentiate this hESC line by the Columbia University Human Embryonic and Human Embryonic Stem Cell Research Committee.

\section{APP ${ }^{\text {Swe }}$ knockin hESC line generation}

The APP ${ }^{\text {Swe }}$ mutation (KM670/671NL) was knocked into both alleles of H9 using CRISPR/Cas9, bi-allelic knockin of $A P P^{S w e}$ has been demonstrated to have increased $A \beta$ production relatively to monoallelic knockin in a control iPSC line (Paquet et al., 2016). In brief, a sgRNA targeting Exon16 of APP was designed and subcloned into the MLM3636 vector, a gift from Keith Joung (Addgene plasmid \# 43860; http://www.addgene.com/43860). A ssDNA HDR template (IDT) was designed in which the APPSwe mutation disrupted the PAM and introduced a de novo Xba1 restriction site. The sgRNA, Cas9-GFP (a gift from Kiran Musunuru (Addgene plasmid \# 44719; http://www.addgene.org/44719/), and ssDNA HDR template were electroporated (Lonza nucleofector) into feeder-free H9 hESCs, followed by cell sorting on GFP signal and plating at low density on MEFs (MTI-GlobalStem). Individual clones were manually picked into a 96 well format, subsequently split into duplicate plates, one of which were used to generate sgDNA as had been done previously (Paquet et al., 2016). For each clone, exon 16 of APP was amplified and initially screened by restriction digest with Xba1 (NEB). Sanger sequencing was used to confirm the mutation, and successful knockin clones were expanded and banked. Potential off-target effects of CRISPR/Cas 9 cleavage were analyzed by Sanger sequencing of the top 5 predicted off-target genomic locations [https://mit.crispr.edu], which demonstrated a lack of indels for multiple clones. One $\mathrm{APP}^{\text {Swe/APP }}{ }^{\text {Swe }}$ knockin line was analyzed and demonstrated to have a normal karyotype (G-banding, Columbia University Clinical Cytology Laboratory), and was used in the present work (Cl. 160). 


\section{Transdifferentiation of hESCs into cortical-like pyramidal neurons}

pLV-TetO-hNGN2-eGFP-Puro was generously provided by Kristen Brennand (Ho et al., 2016) and FUdeltaGW-rtTA was a gift from Konrad Hochedlinger (Addgene plasmid \# 19780; http://www.addgene.org/19780/) (Maherali et al., 2008). Concentrated lentiviruses for these two plasmids were made using Lenti-X concentrator (Takara) as has been done previously (Sun et al., 2019). Permanent doxycycline inducible Ngn2-eGFP lines were generated by reverse infecting $\mathrm{H} 9$ and H9:APP ${ }^{\text {Swe/Swe }}$ hESC lines with NGN2-eGFP and rtTA and subsequent selection by puromycin.

For transdifferentiation of these two lines of hESCs into cortical neurons (iNs; (Zhang et al., 2013)), H9 and H9:APP ${ }^{\text {Swe/Swe }}$ lines were plated at $\sim 165,000$ cells per 12 well-well in N2/B27 medium (Topol et al., 2015) on PEI ( $0.1 \%$ Sigma)/laminin $(10 \mathrm{ug} / \mathrm{mL}$, Biotechne) coated plates, supplemented with $1 \mathrm{ug} / \mathrm{mL}$ doxycycline (Sigma), $10 \mathrm{ng} / \mathrm{mL}$ BDNF and NT3 (Biotechne) and $1 \mathrm{ug} / \mathrm{mL}$ laminin (Biotechne). BDNF, NT3 and laminin were added at similar levels for all subsequent feeds for the duration of the experiment. One day post plating cells were treated with puromycin $(1 \mathrm{ug} / \mathrm{mL}$, Fisher). The following day cells were treated with $2 \mathrm{uM} \mathrm{AraC} \mathrm{(Biotechne)} \mathrm{for} 4$ days, and $100 \mathrm{ng} / \mathrm{mL}$ on subsequent feeds. One week post infection cells were transitioned into BrainPhys (Stem Cell Technologies). Two weeks post infection, 10\% mouse astrocyte conditioned media (ACM; ScienCell) was also added to the medium. Transfection of mitoDsRed2 was done at day 21-23 post infection using Lipofectamine 3000 (Life), and cells were fixed and analyzed 4 days later. A full media change (1/2 saved conditioned media) was performed $\sim 6 \mathrm{~h}$ post transfection. Note that iNs had poor survival from Lipofectamine transfection without either ACM or primary mouse glia present.

\section{Cell Lysis and Immunoprecipitation}

Neuron and differentiated hESC cultures were washed with cold PBS and lysed in Triton Lysis Buffer: 20mM HEPES (pH 7.5), 150mM NaCl, 1 mM EDTA, 1 mM EGTA, 1\% Triton X-100, 0.25M Sucrose, Complete ${ }^{\mathrm{TM}}$ protease inhibitor (Roche), and phosphoSTOP (Roche). Lysates were incubated at $4^{\circ} \mathrm{C}$ for 15 minutes and cleared at $15,000 \times \mathrm{g}$ for 15 minutes at $4^{\circ} \mathrm{C}$. Total protein was normalized using Bio-rad Protein Assay Dye (Bio-rad).

For western blotting of phospho-T172 AMPK and total AMPK, equal amounts of lysates were loaded on a Mini-Protean TGX (4-20\%) SDS-PAGE (Bio-rad). The separated proteins were transferred onto polyvinylidene difluoride membrane (PVDF, Bio-Rad), blocked for 1 hour with blocking buffer containing $5 \%$ fat-free dry milk in TBS-T. Membranes were then incubated overnight at $4{ }^{\circ} \mathrm{C}$ with different primary antibodies diluted in the same blocking buffer. Incubations with HRP conjugated secondary antibodies were performed for 1 hour at room temperature, and visualization was performed by chemiluminescence. For phospho-MFF and MFF immunodetection, equal amounts of lysates $(80 \mu \mathrm{g})$ were immunoprecipitated with MFF or HA (as negative control for specific binding) to enhance MFF proteins. SureBeads ${ }^{\mathrm{TM}}$ Protein A Magnetic Beads were washed three times with lysis buffer and added to equilibrated lysates for 1 hour at $4^{\circ} \mathrm{C}$. The beads were washed 2 times with lysis buffer, two times with lysis buffer without Triton X-100, and then eluted by boiling in SDS lysis buffer for 5 minutes at $95^{\circ} \mathrm{C}$ and resolved by SDS-PAGE (Biorad) gel. The separated proteins were transferred onto polyvinylidene difluoride membrane (PVDF, BioRad), blocked for 1 hour with blocking buffer containing 5\% BSA in Tris-buffered saline solution. Membranes were incubated overnight at $4^{\circ} \mathrm{C}$ with either phospho or total MFF primary antibodies diluted in the same blocking buffer. Clean-Blot ${ }^{\mathrm{TM}}$ IP Detection Kit (Thermo Fisher Scientific) was used to detect the bands of interest without the detection of denatured IgG as MFF protein size is close to both heavy and light chain. After overnight primary antibody incubation, membranes were incubated with Dilute 
Clean-Blot IP Detection Reagent at 1:100 for 1 hour at room temperature and visualization was performed by enhanced chemiluminescence.

For phospho-ULK2 experiments, 293T cells were transfected using Lipofectamine 2000 (Life Technologies) according to manufacturer's instructions with pcDNA3 plasmids encoding flag tagged WT ULK2, flag tagged K39I ULK2 (ULK2 KI) or flag tagged S309A,T441A,S528A,S547A ULK2 (SA4). Where indicated, cells were co-transfected with a constitutively active truncated version of AMPK 1 (caAMPK).

Transfected cell lysates were harvested 40-48 hours post transfection. 1 hour after changing the media with fresh media containing vehicle or $50 \mu \mathrm{M}$ compound 991 (Glixx laboratories, Inc.), cells were washed with cold PBS and lysed in lysis buffer: $20 \mathrm{mM}$ Tris pH 7.5, $150 \mathrm{mM} \mathrm{NaCl}, 1 \mathrm{mM}$ EDTA, 1mM EGTA, 1\% Triton X-100, $2.5 \mathrm{mM}$ pyrophosphate, $50 \mathrm{mM} \mathrm{NaF}, 5 \mathrm{mM}$-glycero-phosphate, $50 \mathrm{nM}$ calyculin A, $1 \mathrm{mM}$ $\mathrm{Na3VO} 4$, and protease inhibitors (Roche). Lysates were incubated at $4^{\circ} \mathrm{C}$ for 15 minutes and cleared at $16,000 \mathrm{~g}$ for 15 minutes at $4^{\circ} \mathrm{C}$. Total protein was normalized using BCA protein kit (Pierce) and lysates were resolved on SDS-PAGE gel. Immunoprecipitations were performed by adding magnetic M2 FLAG beads (Sigma) to equilibrated lysates for 2 hours at $4^{\circ} \mathrm{C}$. The beads were washed three times with lysis buffer and then eluted by boiling in SDS lysis buffer for 5 minutes and resolved by SDS-PAGE.

Antibodies used in the paper are the following: anti-phospho-T172-AMPKa $(40 \mathrm{H} 9,1: 1000$, Cell Signaling); AMPKa1/2 (1:1000, Cell Signaling); MFF (1:1000, Proteintech); phospho-S146/172-MFF (1:1000, Cell Signaling); pAMPK motif (CST, \#5759S); total AMPK $\alpha$ (CST, \#2532), Flag M2 (Sigma, F7425), pAMPK (CST, \#2535), actin (Sigma, A5441). HRP-coupled secondary antibodies to mouse (AP124P) or rabbit (AP132P) were from Millipore. Li-Cor Fluorescence-coupled secondary antibodies to mouse or rabbit were from Li-Cor Biosciences.

\section{Statistics}

Statistical analyses were performed with Prism 6 (GraphPad Software). The statistical test applied for data analysis is indicated in the corresponding figure legend. Experimental groups where all distributions were Gaussian/normal were assessed using the unpaired t-test for two-population comparison, or oneway ANOVA with Dunnett's post hoc test for multiple comparisons. Nonparametric tests including the Mann-Whitney $U$ test for two-population comparison and kruskal-Wallis with Dunn's posttest for multiple comparisons were applied when distributions were not Gaussian. Unless otherwise noted, data are expressed as mean \pm SEM. For dendritic spine and mitochondrial morphology analysis, all data were obtained from at least three independent experiments or at least three individual mice. The test was considered significant when $p<0.05$. For all analyses, the following apply: ${ }^{*} p<0.05 ;{ }^{* *} p<0.01 ;{ }^{* * *} p<0.001$; ${ }^{* * * *} p<0.0001$; ns, not significant with $p>0.05$. 


\section{Figure Legends}

Figure 1. A $\beta 420$ induces dendritic mitochondrial fragmentation and dendritic spine loss within the same time frame.

(A-C') Representative images of primary cortical neurons at 21 days in vitro (DIVs). Neurons express Venus $(A, B, C)$ and mito-DsRed (A', B', C') to assess spine density and mitochondrial morphology, respectively. At 20 DIVs, neurons were treated with either 24 hours of control vehicle, 14 or 24 hours of Aß42o (300-450nM- see Material and Methods for details). High magnification of secondary dendrites is shown below whole neurons shown at low magnification. (D) Quantification of dendritic mitochondrial length. (E) Quantification of dendritic mitochondria density. Dendritic mitochondria density was calculated by summing up the length of all the mitochondria then dividing it by the length of the dendrite examined. (F) Quantification of dendritic spine density calculated by dividing the number of spines by the length of the dendrite examined. All the analysis was done blind to the experimental conditions, and was done by manual counting using FIJI. All of the statistical analyses were performed with Prism 6 (GraphPad Software). Data is represented by box plots displaying minimum to maximum values, with the box denoting $25^{\text {th }}, 50^{\text {th }}$ (median) and $75^{\text {th }}$ percentile from 3 independent experiments. $\mathrm{n}_{\text {control }}=53$ dendrites, 278 mitochondria; $n_{14 h r} A_{\beta 42}=47$ dendrites; 380 mitochondria; $n_{24 h r} A \beta 42=51$ dendrites; 400 mitochondria. Statistical analysis was performed using Kruskal-Wallis test followed by Dunn's post-hoc test in (D-F). The test was considered significant when $p<0.05$ with the following criteria: ${ }^{*} p<0.05$; ${ }^{* *} p<0.01$; ${ }^{* * *} p<0.001 ;{ }^{* * *} p<0.0001$; ns, not significant. Scale bar for high magnification dendritic segments $=2 \mu \mathrm{m}$.

\section{Figure. 2. A $\beta 420$ treatment induces local mitophagy in dendrites}

(A) Dendritic segments (secondary) from primary cortical PNs at 21-25 DIVs electroporated with plasmids encoding mito-mTagBFP2, Lamp1-mEmerald, and RFP-LC3 to visualize mitochondria, lysosomes, and autophagosomes, respectively, using ex utero electroporation on embryos at E15.5. At 21DIVs, neurons were treated with control vehicle or $A \beta 420$ and imaged live with time-lapse microscopy every 15 minutes for 14 hours. The captured image shows dendrites at 0 and 14 hours for both conditions. Yellow box labels the zoomed region shown in ( $\left.\mathbf{A}^{\prime}\right)$ illustrating that in control, there is no significant change of LAMP1+ (lysosomes) and LC3+ (autophagosomes) or LC3/LAMP1 double-positive (autolysosomes) vesicles dynamics or accumulation and no significant change in mitochondrial morphology (see also Movie S1 and S2). In contrast, in dendrites of AB42o treated cortical PNs, sites of LAMP1-LC3 doublepositive autolysosome accumulation often results in loss of mitochondria as indicated by yellow arrow in $A^{\prime}$. We note that some sites do not display loss of mitochondrial signal as within the $14 \mathrm{~h}$ of imaging following A 420 application (red arrow in Fig. 2A). (A') Representative kymograph of mitochondria, lysosomes, and autophagosomes in dendrites from Fig. 2A. (B) Percentage of dendritic segments showing accumulation of both LC3+ autophagosomes, LAMP1+ lysosomes or LC3/ LAMP1-double positive autolysosomes. Data is represented by box plots displaying minimum to maximum values, with the box denoting $25^{\text {th }}, 50^{\text {th }}$ (median) and $75^{\text {th }}$ percentile from 3 independent experiments. $\mathrm{n}_{\text {control }}=15$ neurons; $n_{A \beta 42}=24$ neurons. (C) Random dendritic segments analyzed in B were selected to measure levels of mitophagy. To obtain a spatially specific measurement of mitophagy, intensity line scan measurements were taken across different dendritic regions from the same dendrite. For each dendrite, line scans were drawn in regions without and with LC3 and LAMP1 puncta at 14 hours and the intensity of mitochondrial signal was measured at time point zero and 14 hours. Fold change of mitochondrial intensity at a very spatially focused area was calculated. In control, mitochondrial index across the same dendrite is relatively unchanged in regions with and without LC3 and LAMP1 accumulation, suggesting that mitochondria are not targeted for LC3/LAMP1 degradation. In A 342 treated condition, mitochondrial index along the same dendrite can show significant difference. Only regions of LC3 and LAMP1 
accumulation show mitochondrial loss. Data is represented as bar graph from $\mathrm{n}$ control conditions $=33$ dendrites; $\mathrm{n}_{\mathrm{A} \beta 42 \text { conditions }}=44$ dendrites. One-way ANOVA with Kruskal-Wallis followed by Dunn's posthoc test were applied. The test was considered significant when $p<0.05$ with the following criteria: * $p<0.05 ;{ }^{* *} p<0.01 ;{ }^{* *} p<0.001 ;{ }^{* * * *} p<0.0001$; ns, not significant. Scale bar $=2 \mu \mathrm{m}$.

Figure 3. In AD mouse model, CA1 pyramidal neurons display spatially restricted mitochondrial fragmentation and loss of biomass in distal apical tufts

(A) Representative images of CA1 PN dendrites from different regions of WT control and J20 mice. CA1 PNs were electroporated with pCAG-Venus and pCAG-mito-DsRed by in utero electroporation in E15.5 WT and J20 mouse embryos. Both mouse groups were fixed and imaged at 3 months postnatal (P90). Dendrites from basal, oblique, and distal apical are magnified to show spine density and mitochondrial morphology. The three types of dendrites were characterized as displayed in (B) for both WT and J20 mice. (C-E) Quantification of spine density $(C)$ and individual mitochondrial length (D) and mitochondrial density (fraction of dendritic segment length occupied by mitochondria) ( $E$ ). Data is represented by box plots displaying minimum to maximum values, with the box denoting $25^{\text {th }}, 50^{\text {th }}$ (median) and $75^{\text {th }}$ percentile from at least 3 independent in utero electroporated mice. $\mathrm{N}_{\text {basal } W T}=47$ dendrites, 595 mitochondria; $\mathrm{N}_{\text {basal APP }}=32$ dendrites, 495 mitochondria; $\mathrm{N}_{\text {oblique } W T}=46$ dendrites, 630 mitochondria; $\mathrm{N}_{\text {oblique APP }}=33$ dendrites, 507 mitochondria; $\mathrm{N}_{\text {distal } W T}=39$ dendrites, 284 mitochondria; $\mathrm{N}_{\text {distal APP }}=32$ dendrites; 426 mitochondria. All the analysis was done blind to the experimental conditions, and was manually counted using FIJI. Statistical analysis was performed using Kruskal-Wallis test followed by Dunn's post-hoc test in (C-E). The test was considered significant when $p<0.05$ with the following criteria: ${ }^{*} p<0.05 ;{ }^{* *} p<0.01 ;{ }^{* *} p<0.001 ;{ }^{* * *} p<0.0001 ; n s, p>0.05$. Scale bar $=2 \mu \mathrm{m}$.

Figure 4. Oligomeric $A \beta 42$ induced synaptotoxicity and dendritic mitochondrial fragmentation is AMPK dependent

(A) Dendritic segments (secondary) of primary cortical neuron at 21 DIV. Embryos from AMPKa1/2 double conditional knockout (AMPKa $1^{\mathrm{F} / \mathrm{F}} / 2^{\mathrm{F} / \mathrm{F}}$ ) were ex utero electroporated at $\mathrm{E} 15.5$ with plasmids expressing Venus, mito-DsRed, and either scrambled Cre (control) or Cre recombinase. Neurons were treated at 20 DIV with either control or A 420 for $24 \mathrm{hr}$. (B-D) Quantification of mitochondria length (B), and mitochondrial density (C), and spine density (D) in individual dendritic segments. All the analysis was done blind to the experimental conditions, and was done by manual counting using FIJI. All of the statistical analyses were performed with Prism 6 (GraphPad Software). Data is represented by box plots displaying minimum to maximum values, with the box denoting $25^{\text {th }}, 50^{\text {th }}$ (median) and $75^{\text {th }}$ percentile from 3 independent experiments. $n$-Cre control $=27$ dendrites, 128 mitochondria; $\mathrm{n}_{\text {-Cre }} \mathrm{A} \beta 420=29$ dendrites; 204 mitochondria; $n_{+ \text {Cre control }}=29$ dendrites; 143 mitochondria; $n_{+C r e ~ A \beta 420}=24$ dendrites, 112 mitochondria. Statistical analysis was performed using Kruskal-Wallis test followed by Dunn's post-hoc test in (B-D). The test was considered significant when $p<0.05$ with the following criteria: ${ }^{*} p<0.05$; ${ }^{* *} p<0.01$; ${ }^{* * *} p<0.001 ;{ }^{* * * *} p<0.0001 ; n s$, not significant. Scale bar $=2 \mu \mathrm{m}$.

Figure 5. MFF is required for $A \beta 420$ induced dendritic mitochondrial fragmentation

(A) Representative images of secondary dendritic segments of primary cortical neurons at 21 DIV. Embryos at E15.5 were ex utero electroporated with plasmids encoding Venus, mito-DsRed, and either with control shRNA or an shRNA specific for mouse MFF (MFF shRNA). Neurons were treated at 20 DIV with either control or A 420 for 24 hours. Knockdown of MFF blocks both the synapse and dendritic mitochondrial fragmentation. (B) Quantification of dendritic mitochondrial length, and (C) dendritic mitochondrial density, and (D) spine density. All the analysis was done blind to the experimental 
conditions, and was done by manual counting using FIJI. All of the statistical analyses were performed with Prism 6 (GraphPad Software). Data is represented by box plots displaying minimum to maximum values, with the box denoting $25^{\text {th }}, 50^{\text {th }}$ (median) and $75^{\text {th }}$ percentile from 3 independent experiments. $\mathrm{n}_{\mathrm{PLKO}}$ control $=36$ dendrites, 188 mitochondria; $\mathrm{n}_{\mathrm{PLKO}} \mathrm{A} \beta 420=41$ dendrites; 300 mitochondria; $\mathrm{n}_{\text {MFFshRNA control }}$ = 36 dendrites; 167 mitochondria; $\mathrm{n}_{\text {MFFshRNA A } 420}=30$ dendrites, 125 mitochondria. Statistical analysis was performed using Kruskal-Wallis test followed by Dunn's post-hoc test in (B-D). The test was considered significant when $p<0.05$ with the following criteria: ${ }^{*} p<0.05 ;{ }^{* *} p<0.01 ;{ }^{* * *} p<0.001$; ${ }^{* * \star *} p<0.0001$; ns, not significant. Scale bar for magnified dendritic segments $=2 \mu \mathrm{m}$.

Figure 6. A 3420 induces AMPK-dependent MFF phosphorylation at two serine sites required for A 3420 -dependent dendritic mitochondrial fragmentation and spine loss

(A) Representative images of secondary dendritic segments of primary cortical neurons at 21 DIVs. Embryos at E15.5 were ex utero electroporated with pCAG-Venus and pCAG-mito-DsRed to visualize spines and mitochondria, respectively. Neurons were also electroporated with MFFshRNA along with either low levels of pCAG-MFF-WT or pCAG-MFF-AA mutant constructs and treated with either control or A 420 for 24 hours. Gene replacement (MFFshRNA + MFF AA) with a form of MFF that cannot be phosphorylated by AMPK blocks dendritic mitochondrial fragmentation and spine loss. (B) Quantification of dendritic mitochondrial length, and (C) dendritic mitochondrial density, and (D) spine density. All the analysis were done blind to the experimental conditions, and performed by manual counting using FIJI. Data is represented by box plots displaying minimum to maximum values, with the box denoting $25^{\text {th }}, 50^{\text {th }}$ (median) and $75^{\text {th }}$ percentiles from 3 independent experiments. $\mathrm{n}_{\text {MFFshRNA }+ \text { MFFWT Control }}=31$ dendrites, 166 mitochondria; $\mathrm{n}_{\text {MFFshRNA + MFFWT Aß420 }}=29$ dendrites; 228 mitochondria; $\mathrm{n}_{\text {MFFshRNA + MFFAA control }}=35$ dendrites; 164 mitochondria; $\mathrm{n}_{\text {MFFshRNA + MFFAA AB420 }}=28$ dendrites, 137 mitochondria. Statistical analysis was performed using Kruskal-Wallis test followed by Dunn's post-hoc test in (D-F). The test was considered significant when $p<0.05$ with the following criteria: ${ }^{*} p<0.05 ;{ }^{* *} p<0.01 ;{ }^{* * *} p<0.001 ;{ }^{* * * *} p<0.0001$; ns, not significant. Scale bar in A: $2 \mu \mathrm{m}$.

Figure 7. ULK2 acts in a concerted manner with MFF and leads to loss of mitochondrial biomass following MFF-dependent mitochondrial fragmentation

(A) Representative images of secondary dendritic segments of primary cortical neurons at 21DIV. $\mathrm{ULK}^{\mathrm{F} / \mathrm{F}}$ embryos were ex utero electroporated at E15.5 with plasmids encoding with Venus and mitoDsRed as well as either scrambled Cre (control; -Cre) or Cre recombinase (+Cre). Neurons were treated at 21 DIV with either control or A 420 for 24 hours. Deletion of ULK2 in cortical PNs blocks A 3420 -induced loss of dendritic spines and loss of mitochondrial biomass. (B-D) Quantification of dendritic mitochondrial length (B), dendritic mitochondrial density (C), and dendritic spine density (D). Analysis was done blind to the experimental conditions by manual counting using FIJI. Statistical analysis was performed using one-way ANOVA with Kruskal-Wallis followed by Dunn's post-hoc test in (B-D). Data is represented by box plots displaying minimum to maximum values, with the box denoting $25^{\text {th }}, 50^{\text {th }}$ (median) and $75^{\text {th }}$ percentile from 3 independent experiments. N-CRE DMSOcontrol $=36$ dendrites, 193

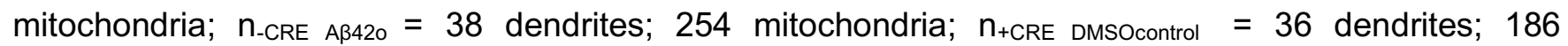
mitochondria; $n_{+C R E ~ A B 420}=35$ dendrites, 435 mitochondria. The test was considered significant when $p<0.05$ with the following criteria: ${ }^{*} p<0.05 ;{ }^{* *} p<0.01 ;{ }^{* *} p<0.001 ;{ }^{* * * *} p<0.0001$; ns, not significant. Scale bar for magnified dendritic segments $=2 \mu \mathrm{m}$. 
Figure 8. AMPK mediated phosphorylation of ULK2 is crucial for oligomeric A 342 induced synaptotoxicity and loss of mitochondrial biomass

(A) Schematic of mULK2 domain structure highlighting the four predicted AMPK-mediated phosphorylation sites (see Fig. S5B) conserved in ULK1 and ULK2. (B) Flag-mULK2 WT or mULK2 4SA (the four conserved phosphorylation sites shown in Fig. S5B are mutated to alanine) is over-expressed in HEK293T cells co-expressing either GST or GST-caAMPKa1(1-312) (constitutively active AMPK). Cells were treated with DMSO or $50 \mu \mathrm{M}$ compound 991 for 1 hour. $5 \mathrm{mg}$ of whole cell lysate was immunoprecipitated with anti-Flag M2 Agarose and immunoblotted with AMPK substrate motif antibody and Flag M2 monoclonal antibody. $25 \mu \mathrm{g}$ of lysates were immunoblotted for $\mathrm{p}$-Thr172 AMPK, total AMPK and actin. (C) Representative high magnification images of dendritic segments showing dendritic spines in the upper panel and mitochondria in the lower panel. ULK2 ${ }^{\mathrm{F} / \mathrm{F}}$ embryos were ex utero electroporated at E15.5 with control vector (-Cre) or Cre recombinase encoding plasmid along with plasmids expressing Venus and mito-DsRed. The Cre-reconstituted (gene replacement) conditions were also coelectroporated with either mULK2 WT (Wild-type), mULK2 KI (K39I) (Kinase inactive) or mULK2 4SA (see Fig. S5). Neurons were treated at 20DIV with either control or A 420 for 24 hours. Abolishing ULK2 kinase-activity (ULK2 KI) or mutating AMPK-mediated phosphorylation sites on ULK2 (ULK2 4SA) can block A 342 oligomer induced synaptotoxicity and loss of mitochondrial biomass. (D) Quantification of dendritic mitochondrial length, and (E) dendritic mitochondrial density, and (F) spine density. Analysis was done blind to the experimental conditions by manual counting using FIJI. Statistical analysis was performed using one-way ANOVA with Kruskal Wallis followed by Dunn's post-hoc test in (D-F). Data is represented by box plots displaying minimum to maximum values, with the box denoting $25^{\text {th }}, 50^{\text {th }}$ (median) and $75^{\text {th }}$ percentile from 3 independent experiments. NuLK2WT DMSOcontrol $=32$ dendrites, 194 mitochondria; nULK2WT Aß42o $=30$ dendrites; 289 mitochondria; nULK2KI DMSOcontrol $=36$ dendrites; 183 mitochondria; nULK2KI Aß420 = 34 dendrites, 449 mitochondria; nULK24SA DMSOcontrol $=34$ dendrites; 198 mitochondria; nULK24SA A $420=32$ dendrites, 472 mitochondria. The test was considered significant when $p<0.05$ with the following criteria: ${ }^{*} p<0.05 ;{ }^{* *} p<0.01 ;{ }^{* *} p<0.001 ;{ }^{* * *} p<0.0001$; ns, not significant. Scale bar for magnified dendritic segments $=2 \mu \mathrm{m}$.

\section{Figure 9. Tau phosphorylation at $\mathbf{S} 262$ is required for $\mathrm{A} \beta 420$-induced dendritic mitochondrial remodeling}

(A) Representative images of secondary dendritic segments of primary cortical PNs at 21 DIV. E15.5 mouse embryos were ex utero electroporated with plasmids encoding Venus, mitoDsRed only or in combination with WT hTau or a non-phosphorylatable mutant hTau-S262A. Neurons were treated at 20 DIV with either vehicle or $A \beta 420$ at $1 \mu \mathrm{M}$ equivalent for 24 hours. Overexpression of hTau in the presence of $A \beta 420$ decreases spine density, mitochondrial length, and mitochondrial density, while over expression of hTauS262A blocked oligomer-induced spine loss and a decrease in density, but not mitochondrial fragmentation. (B) Quantification of dendritic mitochondrial length, (C) dendritic mitochondrial density, and (D) dendritic spine density. All of the analyses were done blind to the experimental conditions, and was done by manual counting in FIJI. All of the statistical analyses were performed in Prism 6 (GraphPad Software). Data is represented by box plots displaying minimum to maximum values, with the box denoting 25th, 50th (median) and 75th percentile from 3-6 independent experiments. $\mathrm{n}_{\text {control }}=81$

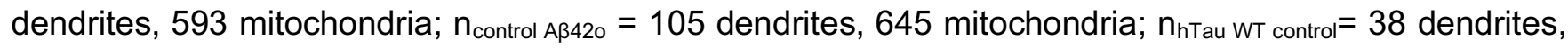
258 mitochondria; $\mathrm{n}_{\mathrm{hTau}} \mathrm{WT}$ A $4420=79$ dendrites, 725 mitochondria; $\mathrm{n}_{\mathrm{hTauS} 262 \mathrm{~A} \text { control }}=81$ dendrites, 555 mitochondria; $n_{\mathrm{hTauS262A} \mathrm{A} 420}=89$ dendrites, 756 mitochondria. Statistical analyses were performed using One-way ANOVA followed by Kruskal-Wallis Multiple Comparisons in (B-D). The test was considered significant when $p<0.05$ with the following criteria: ${ }^{*} p<0.05 ;{ }^{* *} p<0.01$; ${ }^{* * *} p<0.001 ;{ }^{* * *} p<0.0001$; ns, not significant. 


\section{References}

Ando, K., Tomimura, K., Sazdovitch, V., Suain, V., Yilmaz, Z., Authelet, M., Ndjim, M., Vergara, C., Belkouch, M., Potier, M.C., et al. (2016). Level of PICALM, a key component of clathrin-mediated endocytosis, is correlated with levels of phosphotau and autophagy-related proteins and is associated with tau inclusions in AD, PSP and Pick disease. Neurobiol Dis 94, 32-43. 10.1016/j.nbd.2016.05.017

Arbel-Ornath, M., Hudry, E., Boivin, J.R., Hashimoto, T., Takeda, S., Kuchibhotla, K.V., Hou, S., Lattarulo, C.R., Belcher, A.M., Shakerdge, N., et al. (2017). Soluble oligomeric amyloid-beta induces calcium dyshomeostasis that precedes synapse loss in the living mouse brain. Mol Neurodegener 12, 27. 10.1186/s13024-017-0169-9

Arriagada, P.V., Marzloff, K., and Hyman, B.T. (1992). Distribution of Alzheimer-type pathologic changes in nondemented elderly individuals matches the pattern in Alzheimer's disease. Neurology 42, 1681-1688.

Ashrafi, G., and Schwarz, T.L. (2015). PINK1- and PARK2-mediated local mitophagy in distal neuronal axons. Autophagy 11, 187-189. 10.1080/15548627.2014.996021

Buee, L., Bussiere, T., Buee-Scherrer, V., Delacourte, A., and Hof, P.R. (2000). Tau protein isoforms, phosphorylation and role in neurodegenerative disorders. Brain Res Brain Res Rev 33, 95-130.

Burte, F., Carelli, V., Chinnery, P.F., and Yu-Wai-Man, P. (2015). Disturbed mitochondrial dynamics and neurodegenerative disorders. Nat Rev Neurol 11, 11-24. 10.1038/nrneurol.2014.228

Busche, M.A., Chen, X., Henning, H.A., Reichwald, J., Staufenbiel, M., Sakmann, B., and Konnerth, A. (2012). Critical role of soluble amyloid-beta for early hippocampal hyperactivity in a mouse model of Alzheimer's disease. Proc Natl Acad Sci U S A 109, 8740-8745. 10.1073/pnas. 1206171109

Chan, D.C. (2006). Mitochondria: dynamic organelles in disease, aging, and development. Cell 125, 1241-1252. 10.1016/j.cell.2006.06.010

Chan, S.L., Mayne, M., Holden, C.P., Geiger, J.D., and Mattson, M.P. (2000). Presenilin-1 mutations increase levels of ryanodine receptors and calcium release in PC12 cells and cortical neurons. J Biol Chem 275, 18195-18200. 10.1074/jbc.M000040200

Cheong, H., Lindsten, T., Wu, J., Lu, C., and Thompson, C. (2011). Ammonia-induced autophagy is independent of ULK1/ULK2 kinases. PNAS 108, 11121-11126. 10.1073/pnas.1115120108

Cho, D.-H., Nakamura, T., Fang, J., Cieplak, P., Godzik, A., Gu, Z., and Lipton, S. (2009). SNitrosylation of Drp1 Mediates $\beta$-Amyloid Related Mitochondrial Fission and Neuronal Injury. Science 324, 102-105.

Courchet, J., Lewis, T.L., Jr., Lee, S., Courchet, V., Liou, D.Y., Aizawa, S., and Polleux, F. (2013). Terminal axon branching is regulated by the LKB1-NUAK1 kinase pathway via presynaptic mitochondrial capture. Cell 153, 1510-1525. 10.1016/j.cell.2013.05.021

David, D.C., Hauptmann, S., Scherping, I., Schuessel, K., Keil, U., Rizzu, P., Ravid, R., Drose, S., Brandt, U., Muller, W.E., et al. (2005). Proteomic and functional analyses reveal a mitochondrial dysfunction in P301L tau transgenic mice. J Biol Chem 280, 23802-23814. 10.1074/jbc.M500356200

Demuro, A., Mina, E., Kayed, R., Milton, S.C., Parker, I., and Glabe, C.G. (2005). Calcium dysregulation and membrane disruption as a ubiquitous neurotoxic mechanism of soluble amyloid oligomers. J Biol Chem 280, 17294-17300. 10.1074/jbc.M500997200

Du, H., Guo, L., Yan, S., Sosunov, A., McKhann, G., and Yan, S.S. (2010). Early deficits in synaptic mitochondria in an Alzheimer's disease mouse model. PNAS 107, 18670-18675.

DuBoff, B., Gotz, J., and Feany, M.B. (2012). Tau promotes neurodegeneration via DRP1 mislocalization in vivo. Neuron 75, 618-632. 10.1016/j.neuron.2012.06.026

Ducommun, S., Deak, M., Sumpton, D., Ford, R.J., Nunez Galindo, A., Kussmann, M., Viollet, B., Steinberg, G.R., Foretz, M., Dayon, L., et al. (2015). Motif affinity and mass spectrometry proteomic approach for the discovery of cellular AMPK targets: identification of mitochondrial fission factor as a new AMPK substrate. Cell Signal 27, 978-988. 10.1016/j.cellsig.2015.02.008 
Eckert, A., Hauptmann, S., Scherping, I., Meinhardt, J., Rhein, V., Drose, S., Brandt, U., Fandrich, M., Muller, W.E., and Gotz, J. (2008). Oligomeric and fibrillar species of beta-amyloid (A beta 42) both impair mitochondrial function in P301L tau transgenic mice. J Mol Med (Berl) 86, 1255-1267. 10.1007/s00109-008-0391-6

Egan, D.F., Shackelford, D.B., Mihaylova, M.M., Gelino, S., Kohnz, R.A., Mair, W., Vasquez, D.S., Joshi, A., Gwinn, D.M., Taylor, R., et al. (2011). Phosphorylation of ULK1 (hATG1) by AMPactivated protein kinase connects energy sensing to mitophagy. Science 331, 456-461. 10.1126/science.1196371

Fang, E.F., Hou, Y., Palikaras, K., Adriaanse, B.A., Kerr, J.S., Yang, B., Lautrup, S., Hasan-Olive, M.M., Caponio, D., Dan, X., et al. (2019). Mitophagy inhibits amyloid-beta and tau pathology and reverses cognitive deficits in models of Alzheimer's disease. Nat Neurosci 22, 401-412. $10.1038 / \mathrm{s} 41593-018-0332-9$

Garcia, D., and Shaw, R.J. (2017). AMPK: Mechanisms of Cellular Energy Sensing and Restoration of Metabolic Balance. Mol Cell 66, 789-800. 10.1016/j.molcel.2017.05.032

Grienberger, C., Rochefort, N.L., Adelsberger, H., Henning, H.A., Hill, D.N., Reichwald, J., Staufenbiel, M., and Konnerth, A. (2012). Staged decline of neuronal function in vivo in an animal model of Alzheimer's disease. Nat Commun 3, 774. 10.1038/ncomms1783

Guerrier, S., Coutinho-Budd, J., Sassa, T., Gresset, A., Jordan, N.V., Chen, K., Jin, W.L., Frost, A., and Polleux, F. (2009). The F-BAR domain of srGAP2 induces membrane protrusions required for neuronal migration and morphogenesis. Cell 138, 990-1004. 10.1016/j.cell.2009.06.047

Guo, Q., Fu, W., Sopher, B.L., Miller, M.W., Ware, C.B., Martin, G.M., and Mattson, M.P. (1999). Increased vulnerability of hippocampal neurons to excitotoxic necrosis in presenilin-1 mutant knock-in mice. Nat Med 5, 101-106. 10.1038/4789

Hand, R., and Polleux, F. (2011). Neurogenin2 regulates the initial axon guidance of cortical pyramidal neurons projecting medially to the corpus callosum. Neural Dev 6, 30. 10.1186/1749-8104-6-30

Hardie, D.G., Ross, F.A., and Hawley, S.A. (2012). AMPK: a nutrient and energy sensor that maintains energy homeostasis. Nat Rev Mol Cell Biol 13, 251-262. 10.1038/nrm3311

Harnett, M.T., Makara, J.K., Spruston, N., Kath, W.L., and Magee, J.C. (2012). Synaptic amplification by dendritic spines enhances input cooperativity. Nature 491, 599-602. 10.1038/nature11554

Herzig, S., and Shaw, R.J. (2018). AMPK: guardian of metabolism and mitochondrial homeostasis. Nat Rev Mol Cell Biol 19, 121-135. 10.1038/nrm.2017.95

Ho, S.M., Hartley, B.J., Tcw, J., Beaumont, M., Stafford, K., Slesinger, P.A., and Brennand, K.J. (2016). Rapid Ngn2-induction of excitatory neurons from hiPSC-derived neural progenitor cells. Methods 101, 113-124. 10.1016/j.ymeth.2015.11.019

Hong, S., Beja-Glasser, V.F., Nfonoyim, B.M., Frouin, A., Li, S., Ramakrishnan, S., Merry, K.M., Shi, Q., Rosenthal, A., Barres, B.A., et al. (2016). Complement and microglia mediate early synapse loss in Alzheimer mouse models. Science 352, 712-716. 10.1126/science.aad8373

Hsieh, H., Boehm, J., Sato, C., Iwatsubo, T., Tomita, T., Sisodia, S., and Malinow, R. (2006). AMPAR removal underlies Abeta-induced synaptic depression and dendritic spine loss. Neuron 52, 831843. 10.1016/j.neuron.2006.10.035

Itoh, K., Nakamura, K., lijima, M., and Sesaki, H. (2013). Mitochondrial dynamics in neurodegeneration. Trends Cell Biol 23, 64-71. 10.1016/j.tcb.2012.10.006

Ittner, L.M., and Gotz, J. (2011). Amyloid-beta and tau--a toxic pas de deux in Alzheimer's disease. Nat Rev Neurosci 12, 65-72. 10.1038/nrn2967

Ittner, L.M., Ke, Y.D., Delerue, F., Bi, M., Gladbach, A., van Eersel, J., Wolfing, H., Chieng, B.C., Christie, M.J., Napier, I.A., et al. (2010). Dendritic function of tau mediates amyloid-beta toxicity in Alzheimer's disease mouse models. Cell 142, 387-397. 10.1016/j.cell.2010.06.036

Jacobsen, J.S., Wu, C.C., Redwine, J.M., Comery, T.A., Arias, R., Bowlby, M., Martone, R., Morrison, J.H., Pangalos, M.N., Reinhart, P.H., et al. (2006). Early-onset behavioral and synaptic deficits in a mouse model of Alzheimer's disease. Proc Natl Acad Sci U S A 103, 5161-5166. 10.1073/pnas.0600948103

Jin, M., Shepardson, N., Yang, T., Chen, G., Walsh, D., and Selkoe, D.J. (2011). Soluble amyloid betaprotein dimers isolated from Alzheimer cortex directly induce Tau hyperphosphorylation and neuritic degeneration. Proc Natl Acad Sci U S A 108, 5819-5824. 10.1073/pnas.1017033108 
Kim, J., Kundu, M., Viollet, B., and Guan, K.L. (2011). AMPK and mTOR regulate autophagy through direct phosphorylation of Ulk1. Nat Cell Biol 13, 132-141. 10.1038/ncb2152

Kuchibhotla, K.V., Goldman, S.T., Lattarulo, C.R., Wu, H.Y., Hyman, B.T., and Bacskai, B.J. (2008). Abeta plaques lead to aberrant regulation of calcium homeostasis in vivo resulting in structural and functional disruption of neuronal networks. Neuron 59, 214-225. 10.1016/j.neuron.2008.06.008

Lacor, P.N., Buniel, M.C., Chang, L., Fernandez, S.J., Gong, Y., Viola, K.L., Lambert, M.P., Velasco, P.T., Bigio, E.H., Finch, C.E., et al. (2004). Synaptic targeting by Alzheimer's-related amyloid beta oligomers. J Neurosci 24, 10191-10200. 10.1523/JNEUROSCI.3432-04.2004

Lacor, P.N., Buniel, M.C., Furlow, P.W., Clemente, A.S., Velasco, P.T., Wood, M., Viola, K.L., and Klein, W.L. (2007). Abeta oligomer-induced aberrations in synapse composition, shape, and density provide a molecular basis for loss of connectivity in Alzheimer's disease. J Neurosci 27 , 796-807. 10.1523/JNEUROSCI.3501-06.2007

Lasagna-Reeves, C.A., de Haro, M., Hao, S., Park, J., Rousseaux, M.W., Al-Ramahi, I., Jafar-Nejad, P., Vilanova-Velez, L., See, L., De Maio, A., et al. (2016). Reduction of Nuak1 Decreases Tau and Reverses Phenotypes in a Tauopathy Mouse Model. Neuron 92, 407-418. 10.1016/j.neuron.2016.09.022

Lee, J.H., McBrayer, M.K., Wolfe, D.M., Haslett, L.J., Kumar, A., Sato, Y., Lie, P.P., Mohan, P., Coffey, E.E., Kompella, U., et al. (2015). Presenilin 1 Maintains Lysosomal $\mathrm{Ca}(2+)$ Homeostasis via TRPML1 by Regulating vATPase-Mediated Lysosome Acidification. Cell Rep 12, 1430-1444. 10.1016/j.celrep.2015.07.050

Lerdkrai, C., Asavapanumas, N., Brawek, B., Kovalchuk, Y., Mojtahedi, N., Olmedillas Del Moral, M., and Garaschuk, O. (2018). Intracellular $\mathrm{Ca}(2+)$ stores control in vivo neuronal hyperactivity in a mouse model of Alzheimer's disease. Proc Natl Acad Sci U S A. 10.1073/pnas. 1714409115

Lewis, T.L., Jr., Courchet, J., and Polleux, F. (2013). Cell biology in neuroscience: Cellular and molecular mechanisms underlying axon formation, growth, and branching. J Cell Biol 202, 837848. 10.1083/jcb.201305098

Lewis, T.L., Jr., Kwon, S.K., Lee, A., Shaw, R., and Polleux, F. (2018). MFF-dependent mitochondrial fission regulates presynaptic release and axon branching by limiting axonal mitochondria size. Nat Commun 9, 5008. 10.1038/s41467-018-07416-2

Lewis, T.L., Jr., Turi, G.F., Kwon, S.K., Losonczy, A., and Polleux, F. (2016). Progressive Decrease of Mitochondrial Motility during Maturation of Cortical Axons In Vitro and In Vivo. Curr Biol 26, 26022608. 10.1016/j.cub.2016.07.064

Ma, T., Chen, Y., Vingtdeux, V., Zhao, H., Viollet, B., Marambaud, P., and Klann, E. (2014). Inhibition of AMP-activated protein kinase signaling alleviates impairments in hippocampal synaptic plasticity induced by amyloid beta. J Neurosci 34, 12230-12238. 10.1523/JNEUROSCI.1694-14.2014

Maday, S., and Holzbaur, E.L. (2016). Compartment-Specific Regulation of Autophagy in Primary Neurons. J Neurosci 36, 5933-5945. 10.1523/JNEUROSCI.4401-15.2016

Maherali, N., Ahfeldt, T., Rigamonti, A., Utikal, J., Cowan, C., and Hochedlinger, K. (2008). A highefficiency system for the generation and study of human induced pluripotent stem cells. Cell Stem Cell 3, 340-345. 10.1016/j.stem.2008.08.003

Mairet-Coello, G., Courchet, J., Pieraut, S., Courchet, V., Maximov, A., and Polleux, F. (2013). The CAMKK2-AMPK kinase pathway mediates the synaptotoxic effects of Abeta oligomers through Tau phosphorylation. Neuron 78, 94-108. 10.1016/j.neuron.2013.02.003

Mairet-Coello, G., and Polleux, F. (2014). Involvement of 'stress-response' kinase pathways in Alzheimer's disease progression. Curr Opin Neurobiol 27, 110-117. 10.1016/j.conb.2014.03.011

Malinow, R. (2012). New developments on the role of NMDA receptors in Alzheimer's disease. Curr Opin Neurobiol 22, 559-563. 10.1016/j.conb.2011.09.001

Manczak, M., and Reddy, P.H. (2012). Abnormal interaction between the mitochondrial fission protein Drp1 and hyperphosphorylated tau in Alzheimer's disease neurons: implications for mitochondrial dysfunction and neuronal damage. Hum Mol Genet 21, 2538-2547. 10.1093/hmg/dds072

Marinangeli, C., Didier, S., Ahmed, T., Caillerez, R., Domise, M., Laloux, C., Begard, S., Carrier, S., Colin, M., Marchetti, P., et al. (2018). AMP-Activated Protein Kinase Is Essential for the Maintenance of Energy Levels during Synaptic Activation. iScience 9, 1-13.

10.1016/j.isci.2018.10.006 
Masliah, E., Mallory, M., Alford, M., DeTeresa, R., Hansen, L.A., McKeel, D.W., Jr., and Morris, J.C. (2001). Altered expression of synaptic proteins occurs early during progression of Alzheimer's disease. Neurology 56, 127-129.

Miyagawa, T., Ebinuma, I., Morohashi, Y., Hori, Y., Young Chang, M., Hattori, H., Maehara, T., Yokoshima, S., Fukuyama, T., Tsuji, S., et al. (2016). BIN1 regulates BACE1 intracellular trafficking and amyloid-beta production. Hum Mol Genet 25, 2948-2958. 10.1093/hmg/ddw146

Mizushima, N. (2007). Autophagy: process and function. Genes Dev 21, 2861-2873. 10.1101/gad.1599207

Moolman, D.L., Vitolo, O.V., Vonsattel, J.P., and Shelanski, M.L. (2004). Dendrite and dendritic spine alterations in Alzheimer models. J Neurocytol 33, 377-387.

10.1023/B:NEUR.0000044197.83514.64

Morel, E., Chamoun, Z., Lasiecka, Z.M., Chan, R.B., Williamson, R.L., Vetanovetz, C., Dall'Armi, C., Simoes, S., Point Du Jour, K.S., McCabe, B.D., et al. (2013). Phosphatidylinositol-3-phosphate regulates sorting and processing of amyloid precursor protein through the endosomal system. Nat Commun 4, 2250. 10.1038/ncomms3250

Mucke, L., Maslia, E., Yu, G.Q., Mallory, M., Rockenstein, E., Tatsuno, G., Hu, K., Kholodenko, D., Johnson-Wood, K., and McConlogue, L. (2000a). High-level neuronal expression of abeta 1-42 in wild-type human amyloid protein precursor transgenic mice synaptotoxicity without plaque formation. J Neurosci 20, 4050-4058.

Mucke, L., Masliah, E., Yu, G.Q., Mallory, M., Rockenstein, E.M., Tatsuno, G., Hu, K., Kholodenko, D., Johnson-Wood, K., and McConlogue, L. (2000b). High-level neuronal expression of abeta 1-42 in wild-type human amyloid protein precursor transgenic mice: synaptotoxicity without plaque formation. J Neurosci 20, 4050-4058.

Nixon, R.A. (2005). Endosome function and dysfunction in Alzheimer's disease and other neurodegenerative diseases. Neurobiol Aging 26, 373-382. 10.1016/j.neurobiolaging.2004.09.018

Nixon, R.A. (2013). The role of autophagy in neurodegenerative disease. Nat Med 19, 983-997. 10.1038/nm.3232

Palop, J.J., Chin, J., Roberson, E.D., Wang, J., Thwin, M.T., Bien-Ly, N., Yoo, J., Ho, K.O., Yu, G.Q., Kreitzer, A., et al. (2007). Aberrant excitatory neuronal activity and compensatory remodeling of inhibitory hippocampal circuits in mouse models of Alzheimer's disease. Neuron 55, 697-711. 10.1016/j.neuron.2007.07.025

Palop, J.J., and Mucke, L. (2010). Amyloid-beta-induced neuronal dysfunction in Alzheimer's disease: from synapses toward neural networks. Nat Neurosci 13, 812-818. 10.1038/nn.2583

Paquet, D., Kwart, D., Chen, A., Sproul, A., Jacob, S., Teo, S., Olsen, K.M., Gregg, A., Noggle, S., and Tessier-Lavigne, M. (2016). Efficient introduction of specific homozygous and heterozygous mutations using CRISPR/Cas9. Nature 533, 125-129. 10.1038/nature17664

Perez-Cruz, C., Nolte, M.W., van Gaalen, M.M., Rustay, N.R., Termont, A., Tanghe, A., Kirchhoff, F., and Ebert, U. (2011). Reduced spine density in specific regions of CA1 pyramidal neurons in two transgenic mouse models of Alzheimer's disease. J Neurosci 31, 3926-3934.

10.1523/JNEUROSCI.6142-10.2011

Pozueta, J., Lefort, R., Ribe, E.M., Troy, C.M., Arancio, O., and Shelanski, M. (2013). Caspase-2 is required for dendritic spine and behavioural alterations in J20 APP transgenic mice. Nat Commun 4, 1939. 10.1038/ncomms2927

Qu, X., Yuan, F.N., Corona, C., Pasini, S., Pero, M.E., Gundersen, G.G., Shelanski, M.L., and Bartolini, F. (2017). Stabilization of dynamic microtubules by mDia1 drives Tau-dependent Abeta1-42 synaptotoxicity. J Cell Biol 216, 3161-3178. 10.1083/jcb.201701045

Rhein, V., Song, X., Wiesner, A., Ittner, L.M., Baysang, G., Meier, F., Ozmen, L., Bluethmann, H., Drose, S., Brandt, U., et al. (2009). Amyloid-beta and tau synergistically impair the oxidative phosphorylation system in triple transgenic Alzheimer's disease mice. Proc Natl Acad Sci U S A 106, 20057-20062. 10.1073/pnas.0905529106

Roberson, E.D., Halabisky, B., Yoo, J.W., Yao, J., Chin, J., Yan, F., Wu, T., Hamto, P., Devidze, N., Yu, G.Q., et al. (2011). Amyloid-beta/Fyn-induced synaptic, network, and cognitive impairments depend on tau levels in multiple mouse models of Alzheimer's disease. J Neurosci 31, 700-711. 10.1523/JNEUROSCI.4152-10.2011 
Roberson, E.D., Scearce-Levie, K., Palop, J.J., Yan, F., Cheng, I.H., Wu, T., Gerstein, H., Yu, G.Q., and Mucke, L. (2007). Reducing endogenous tau ameliorates amyloid beta-induced deficits in an Alzheimer's disease mouse model. Science 316, 750-754. 10.1126/science.1141736

Schon, E.A., and Przedborski, S. (2011). Mitochondria: the next (neurode)generation. Neuron 70, 10331053. 10.1016/j.neuron.2011.06.003

Schwarz, T.L. (2013). Mitochondrial trafficking in neurons. Cold Spring Harb Perspect Biol 5. 10.1101/cshperspect.a011304

Serrano-Pozo, A., Frosch, M.P., Masliah, E., and Hyman, B.T. (2011). Neuropathological alterations in Alzheimer disease. Cold Spring Harb Perspect Med 1, a006189. 10.1101/cshperspect.a006189

Shankar, G.M., Bloodgood, B.L., Townsend, M., Walsh, D.M., Selkoe, D.J., and Sabatini, B.L. (2007). Natural oligomers of the Alzheimer amyloid-beta protein induce reversible synapse loss by modulating an NMDA-type glutamate receptor-dependent signaling pathway. J Neurosci 27, 28662875. 10.1523/JNEUROSCI.4970-06.2007

Shankar, G.M., Li, S., Mehta, T.H., Garcia-Munoz, A., Shepardson, N.E., Smith, I., Brett, F.M., Farrell, M.A., Rowan, M.J., Lemere, C.A., et al. (2008). Amyloid-beta protein dimers isolated directly from Alzheimer's brains impair synaptic plasticity and memory. Nat Med 14, 837-842. 10.1038/nm1782

Sheng, M., Sabatini, B.L., and Sudhof, T.C. (2012). Synapses and Alzheimer's disease. Cold Spring Harb Perspect Biol 4. 10.1101/cshperspect.a005777

Siskova, Z., Justus, D., Kaneko, H., Friedrichs, D., Henneberg, N., Beutel, T., Pitsch, J., Schoch, S., Becker, A., von der Kammer, H., et al. (2014). Dendritic structural degeneration is functionally linked to cellular hyperexcitability in a mouse model of Alzheimer's disease. Neuron 84, 1023-1033. 10.1016/j.neuron.2014.10.024

Small, S.A., Schobel, S.A., Buxton, R.B., Witter, M.P., and Barnes, C.A. (2011). A pathophysiological framework of hippocampal dysfunction in ageing and disease. Nat Rev Neurosci 12, 585-601. 10.1038/nrn3085

Son, S.M., Jung, E.S., Shin, H.J., Byun, J., and Mook-Jung, I. (2012). Abeta-induced formation of autophagosomes is mediated by RAGE-CaMKKbeta-AMPK signaling. Neurobiol Aging 33, 1006 e1011-1023. 10.1016/j.neurobiolaging.2011.09.039

Sorrentino, V., Romani, M., Mouchiroud, L., Beck, J.S., Zhang, H., D'Amico, D., Moullan, N., Potenza, F., Schmid, A.W., Rietsch, S., et al. (2017). Enhancing mitochondrial proteostasis reduces amyloidbeta proteotoxicity. Nature 552, 187-193. 10.1038/nature25143

Stutzmann, G.E., Caccamo, A., LaFerla, F.M., and Parker, I. (2004). Dysregulated IP3 signaling in cortical neurons of knock-in mice expressing an Alzheimer's-linked mutation in presenilin1 results in exaggerated $\mathrm{Ca} 2+$ signals and altered membrane excitability. J Neurosci 24, 508-513. 10.1523/JNEUROSCI.4386-03.2004

Sun, J., Carlson-Stevermer, J., Das, U., Shen, M., Delenclos, M., Snead, A.M., Koo, S.Y., Wang, L., Qiao, D., Loi, J., et al. (2019). CRISPR/Cas9 editing of APP C-terminus attenuates beta-cleavage and promotes alpha-cleavage. Nat Commun 10, 53. 10.1038/s41467-018-07971-8

Szczurkowska, J., Cwetsch, A.W., dal Maschio, M., Ghezzi, D., Ratto, G.M., and Cancedda, L. (2016). Targeted in vivo genetic manipulation of the mouse or rat brain by in utero electroporation with a triple-electrode probe. Nat Protoc 11, 399-412. 10.1038/nprot.2016.014

Terry, R.D., Masliah, E., Salmon, D.P., Butters, N., DeTeresa, R., Hill, R., Hansen, L.A., and Katzman, R. (1991). Physical basis of cognitive alterations in Alzheimer's disease: synapse loss is the major correlate of cognitive impairment. Ann Neurol 30, 572-580. 10.1002/ana.410300410

Thornton, C., Bright, N.J., Sastre, M., Muckett, P.J., and Carling, D. (2011). AMP-activated protein kinase (AMPK) is a tau kinase, activated in response to amyloid beta-peptide exposure. Biochem $\mathrm{J}$ 434, 503-512. 10.1042/BJ20101485

Tokumitsu, H., Inuzuka, H., Ishikawa, Y., Ikeda, M., Saji, I., and Kobayashi, R. (2002). STO-609, a specific inhibitor of the $\mathrm{Ca}(2+) /$ calmodulin-dependent protein kinase kinase. J Biol Chem 277 , 15813-15818. 10.1074/jbc.M201075200

Topol, A., Tran, N.N., and Brennand, K.J. (2015). A guide to generating and using hiPSC derived NPCs for the study of neurological diseases. J Vis Exp, e52495. 10.3791/52495 
Toyama, E., Herzig, S., Courchet, J., Lewis Jr., T., Loson, O., Hellberg, K., Young, N., Chen, H., Polleux, F., Chan, D.C., et al. (2016). AMP-activated protein kinase mediates mitochondrial fission in response to energy stress. Science 351, 275-281.

Udayar, V., Buggia-Prevot, V., Guerreiro, R.L., Siegel, G., Rambabu, N., Soohoo, A.L., Ponnusamy, M., Siegenthaler, B., Bali, J., Aesg, et al. (2013). A paired RNAi and RabGAP overexpression screen identifies Rab11 as a regulator of beta-amyloid production. Cell Rep 5, 1536-1551. 10.1016/j.celrep.2013.12.005

Uytterhoeven, V., Lauwers, E., Maes, I., Miskiewicz, K., Melo, M.N., Swerts, J., Kuenen, S., Wittocx, R., Corthout, N., Marrink, S.J., et al. (2015). Hsc70-4 Deforms Membranes to Promote Synaptic Protein Turnover by Endosomal Microautophagy. Neuron 88, 735-748. 10.1016/j.neuron.2015.10.012

Vingtdeux, V., Davies, P., Dickson, D.W., and Marambaud, P. (2011). AMPK is abnormally activated in tangle- and pre-tangle-bearing neurons in Alzheimer's disease and other tauopathies. Acta Neuropathol 121, 337-349. 10.1007/s00401-010-0759-x

Wang, X., Su, B., Lee, H.G., Li, X., Perry, G., Smith, M.A., and Zhu, X. (2009a). Impaired balance of mitochondrial fission and fusion in Alzheimer's disease. J Neurosci 29, 9090-9103. 10.1523/JNEUROSCI.1357-09.2009

Wang, X., Su, B., Siedlak, S., Moreira, P., Fujioka, H., Wang, Y., Casadesus, G., and Zhu, X. (2008). Amyloid Beta overproduction causes abnormal mitochondrial dynamics via differential modulation of mitochondrial fission/fusion proteins. PNAS 105, 19318-19323.

Wang, X., Su, B., Zheng, L., Perry, G., Smith, M.A., and Zhu, X. (2009b). The role of abnormal mitochondrial dynamics in the pathogenesis of Alzheimer's disease. J Neurochem 109 Suppl 1, 153-159. 10.1111/j.1471-4159.2009.05867.x

Williams, T., Courchet, J., Viollet, B., Brenman, J.E., and Polleux, F. (2011). AMP-activated protein kinase (AMPK) activity is not required for neuronal development but regulates axogenesis during metabolic stress. Proc Natl Acad Sci U S A 108, 5849-5854. 10.1073/pnas.1013660108

Xie, H., Guan, J., Borrelli, L.A., Xu, J., Serrano-Pozo, A., and Bacskai, B.J. (2013). Mitochondrial alterations near amyloid plaques in an Alzheimer's disease mouse model. J Neurosci 33, 1704217051. 10.1523/JNEUROSCI.1836-13.2013

Yoon, S.O., Park, D.J., Ryu, J.C., Ozer, H.G., Tep, C., Shin, Y.J., Lim, T.H., Pastorino, L., Kunwar, A.J., Walton, J.C., et al. (2012). JNK3 perpetuates metabolic stress induced by Abeta peptides. Neuron 75, 824-837. 10.1016/j.neuron.2012.06.024

Youle, R.J., and van der Bliek, A.M. (2012). Mitochondrial fission, fusion, and stress. Science 337, 1062-1065. 10.1126/science.1219855

Zhang, L., Trushin, S., Christensen, T.A., Bachmeier, B.V., Gateno, B., Schroeder, A., Yao, J., Itoh, K., Sesaki, H., Poon, W.W., et al. (2016). Altered brain energetics induces mitochondrial fission arrest in Alzheimer's Disease. Sci Rep 6, 18725. 10.1038/srep18725

Zhang, Y., Pak, C., Han, Y., Ahlenius, H., Zhang, Z., Chanda, S., Marro, S., Patzke, C., Acuna, C., Covy, J., et al. (2013). Rapid single-step induction of functional neurons from human pluripotent stem cells. Neuron 78, 785-798. 10.1016/j.neuron.2013.05.029 


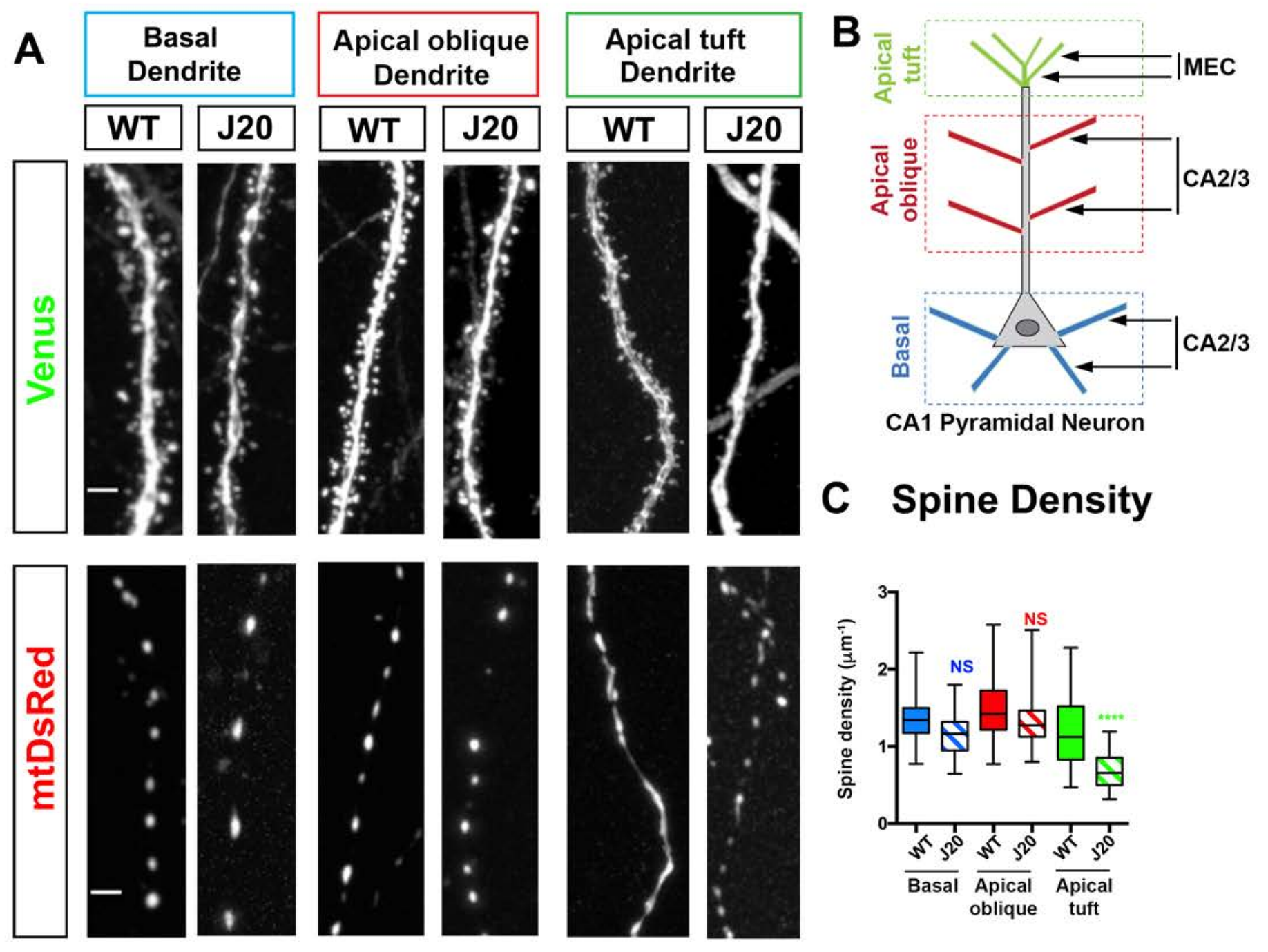

D Mitochondria Length

E Mitochondria Density
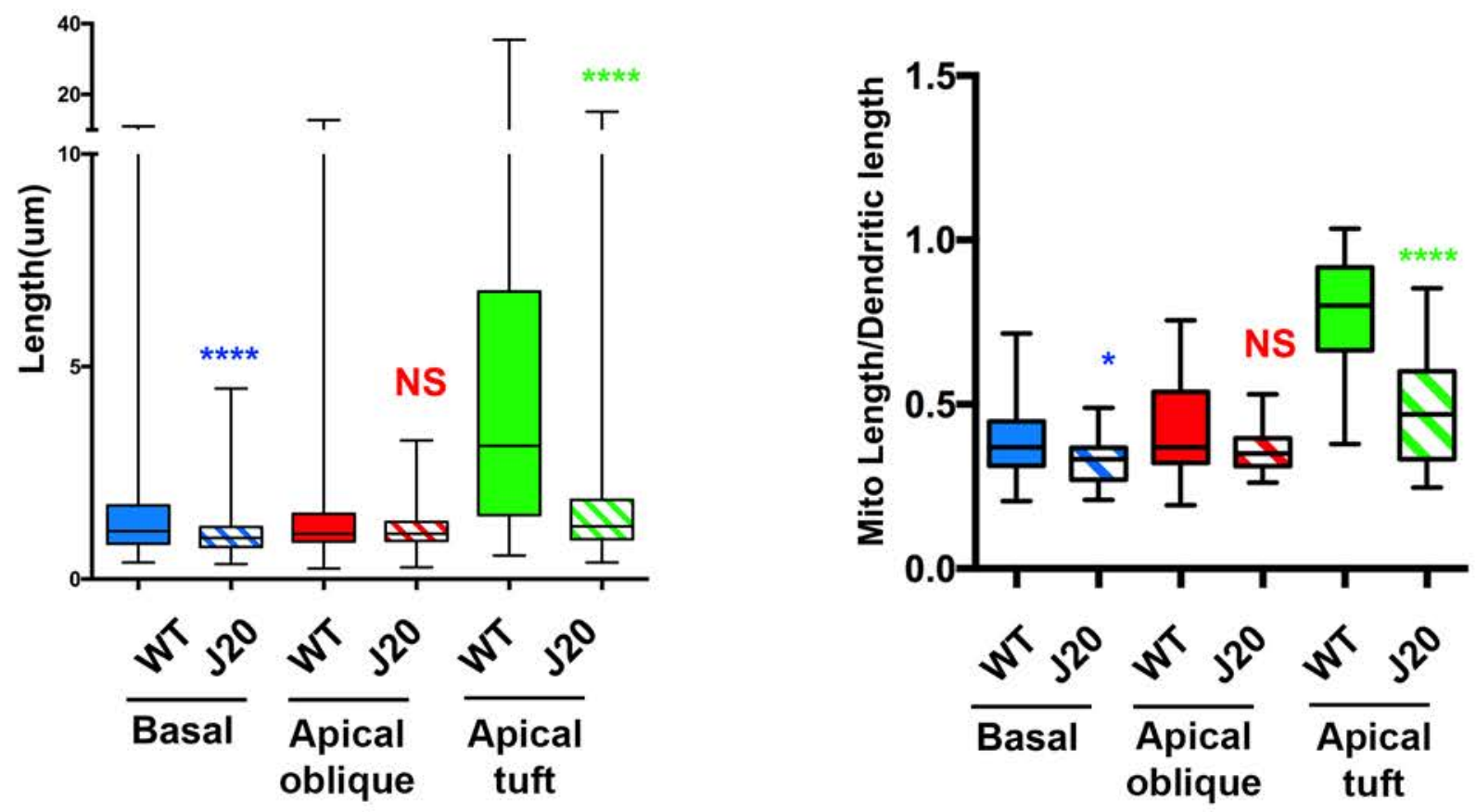

Lee, Kondapalli et al.

Figure 1 

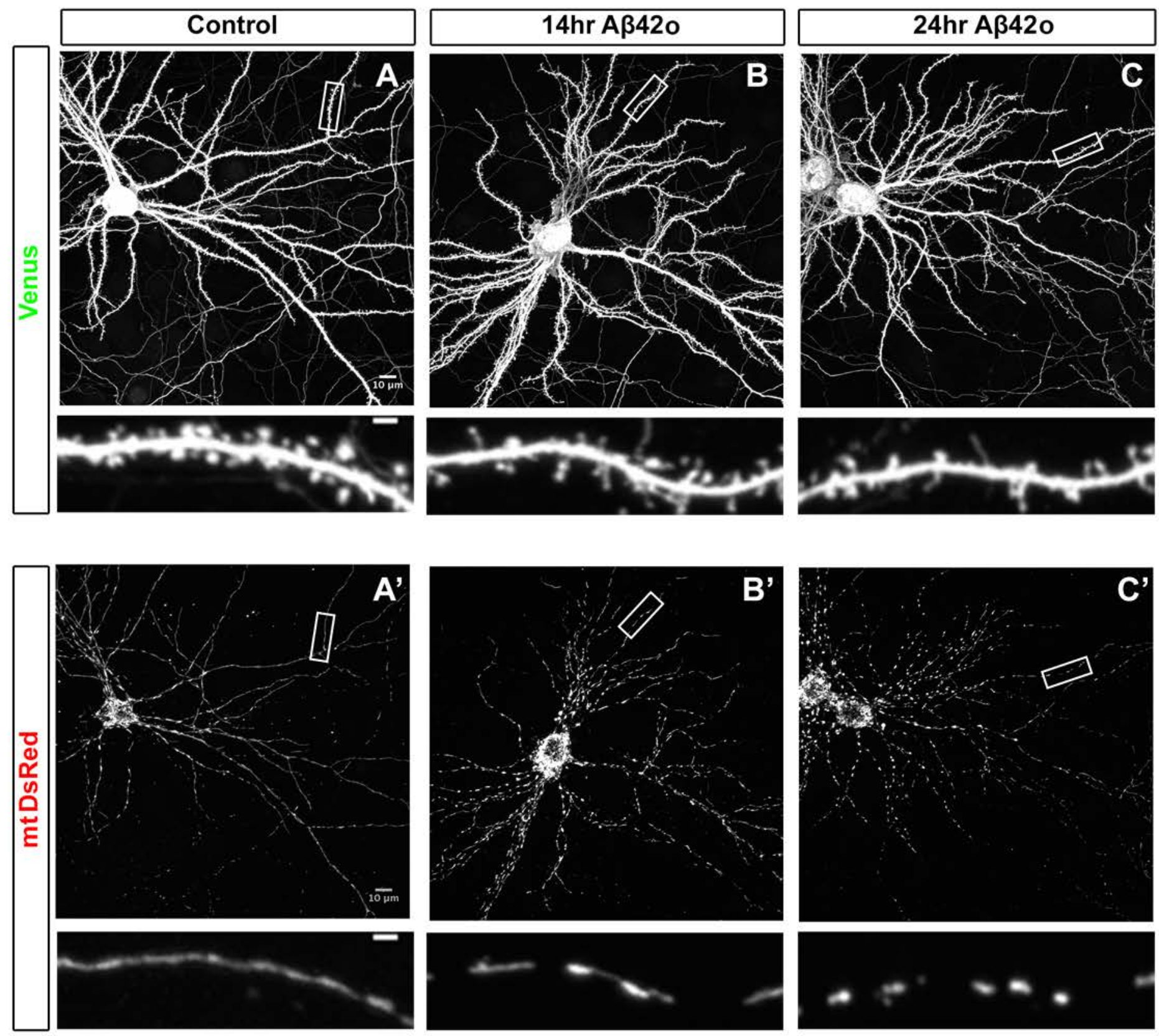

D Mitochondria Length

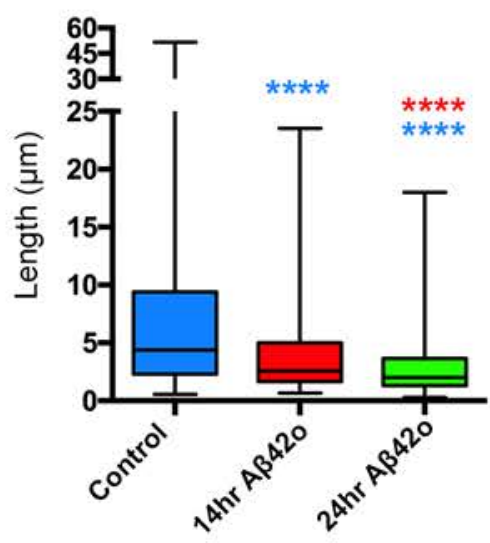

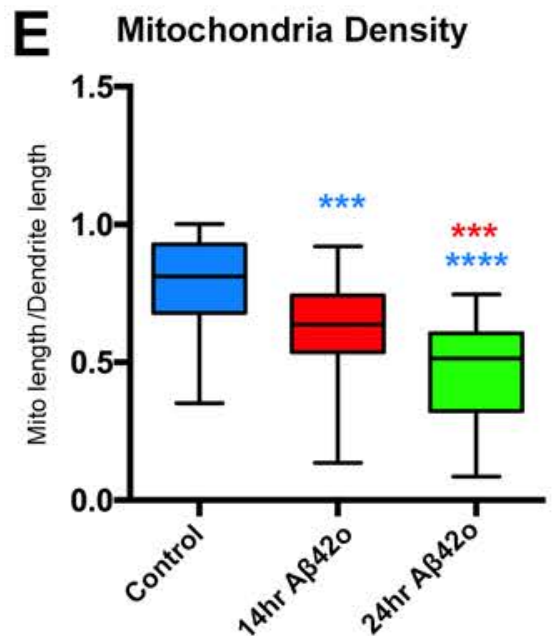
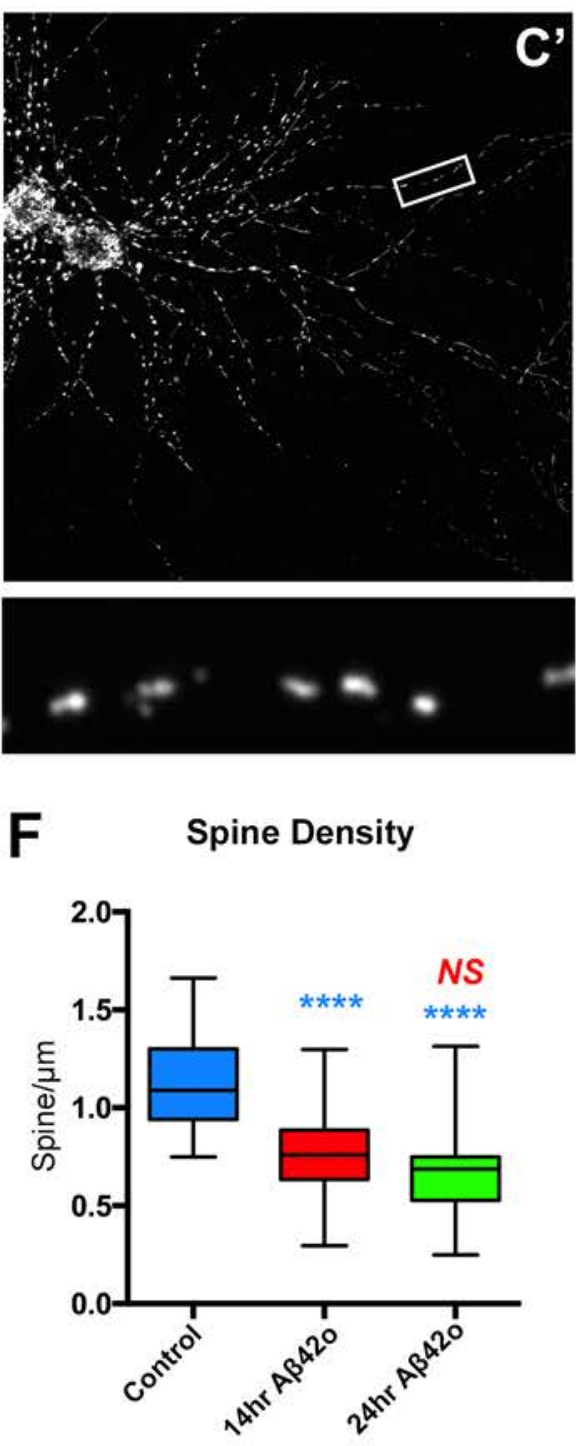

Lee, Kondapalli et al. Figure 2 
A
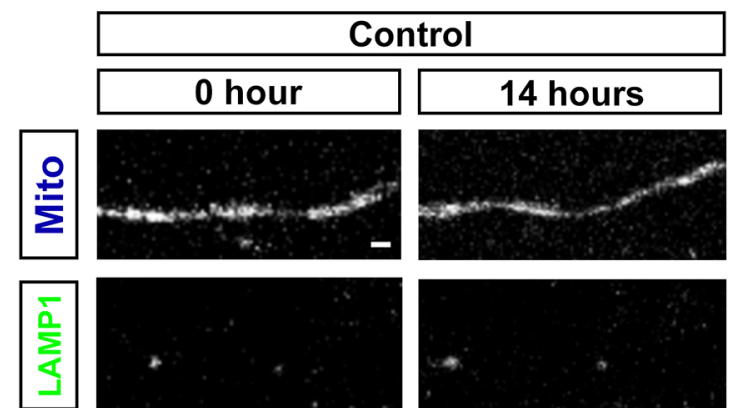

ณ

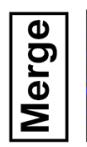

$\mathbf{A}^{\prime}$
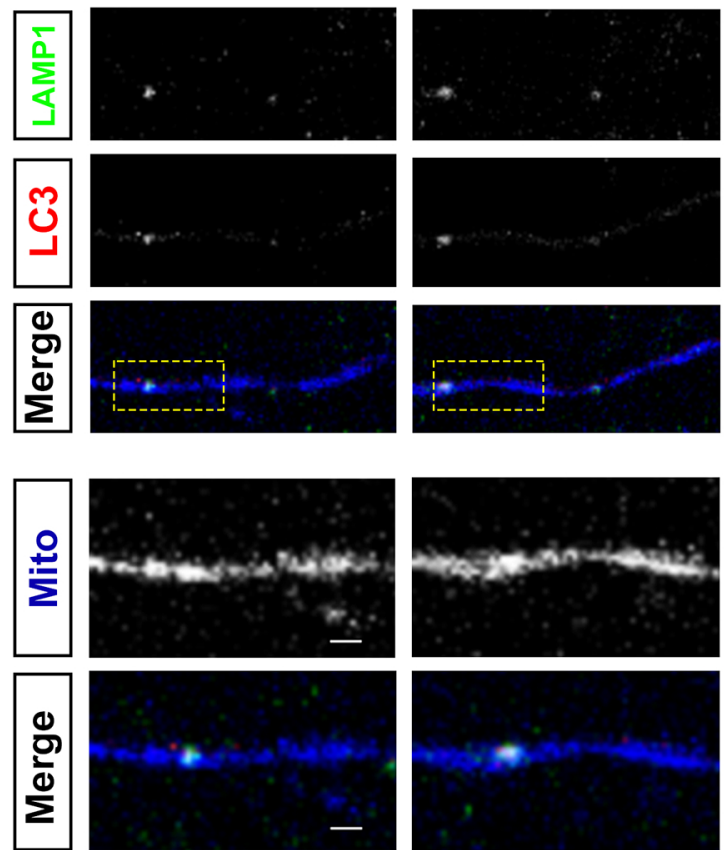
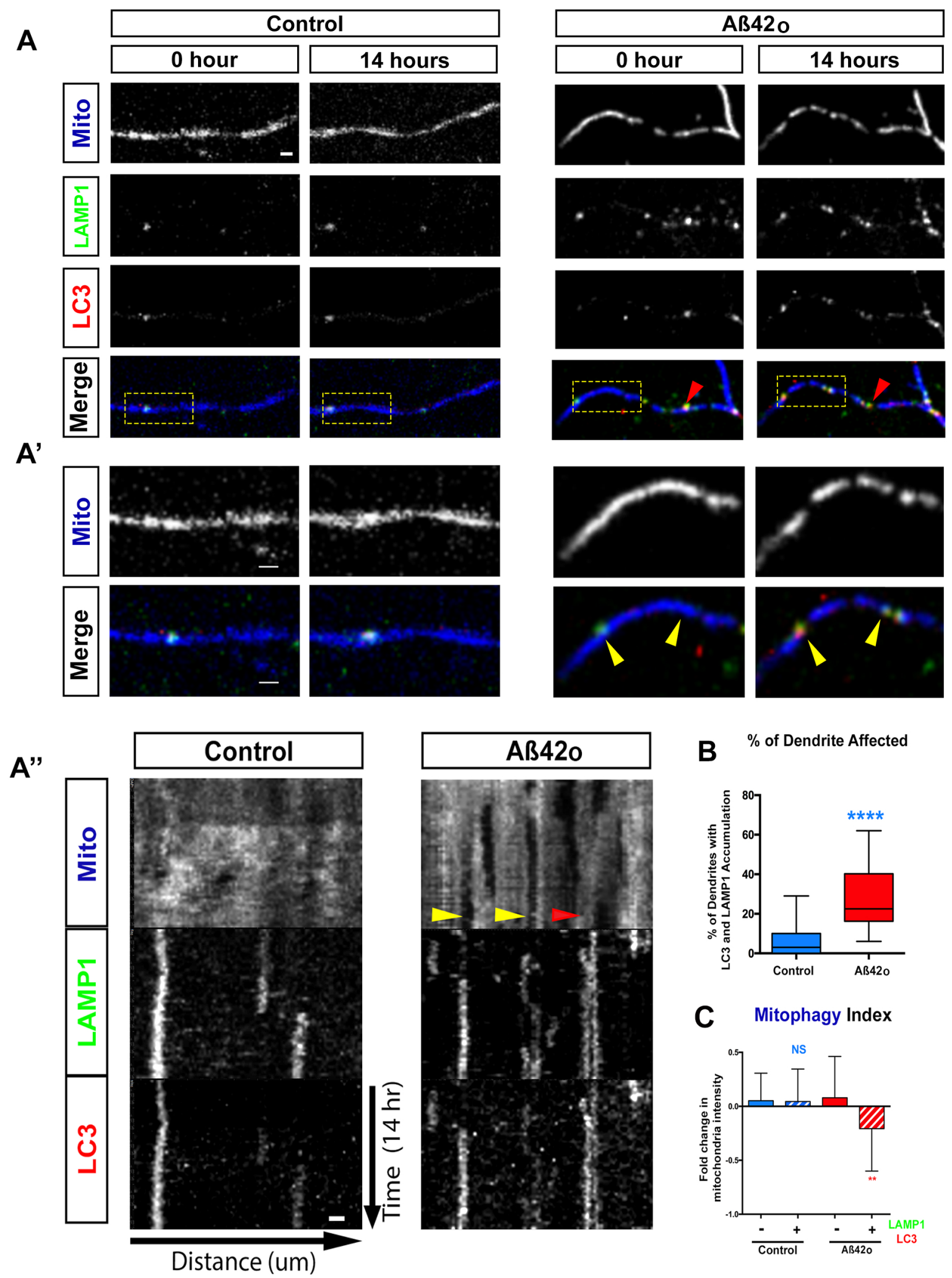

B $\%$ of Dendrite Affected

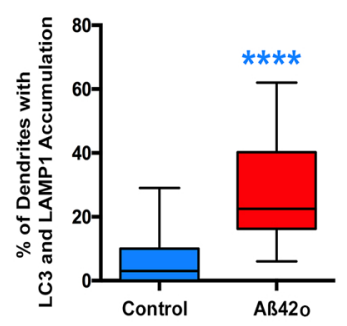

C Mitophagy Index

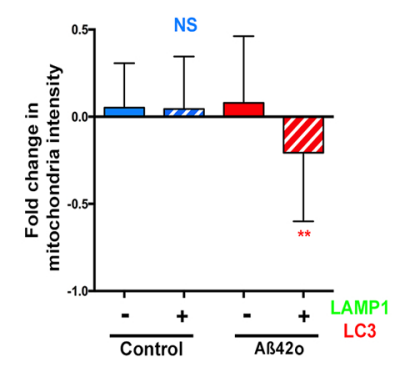

Lee, Kondapalli et al. Figure 3 

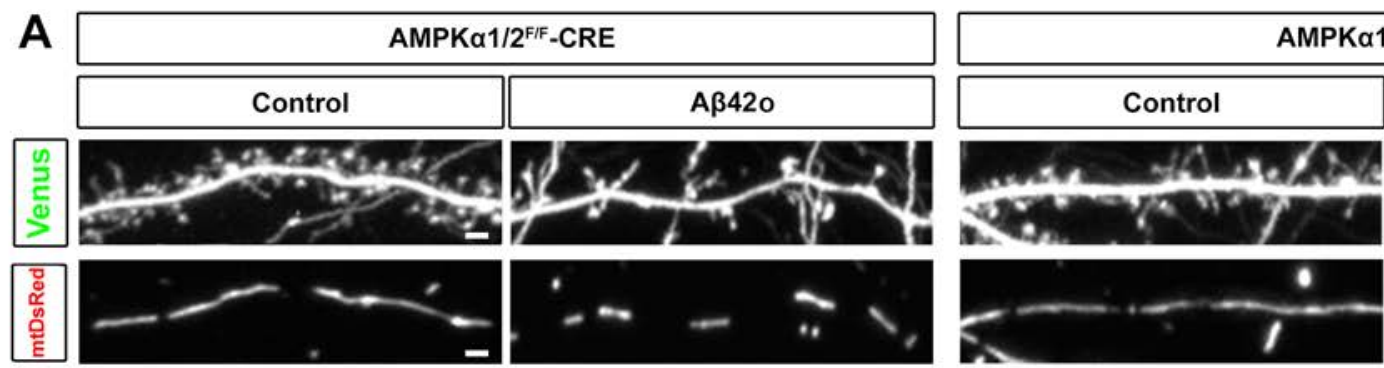

B Mitochondria Length

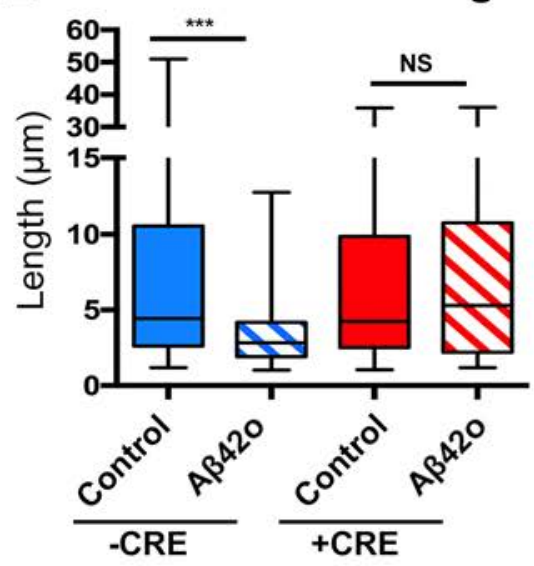

C Mitochondria Density

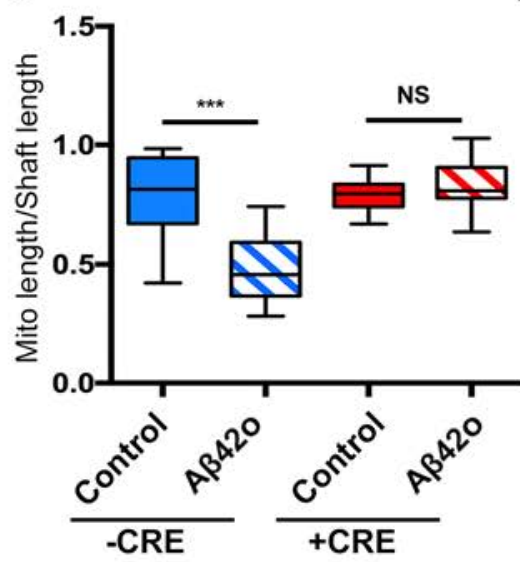

D Spine Density

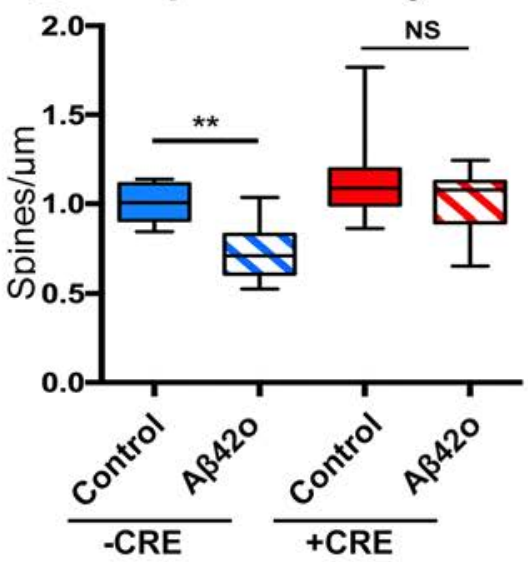

Lee, Kondapalli et al. Figure 4 


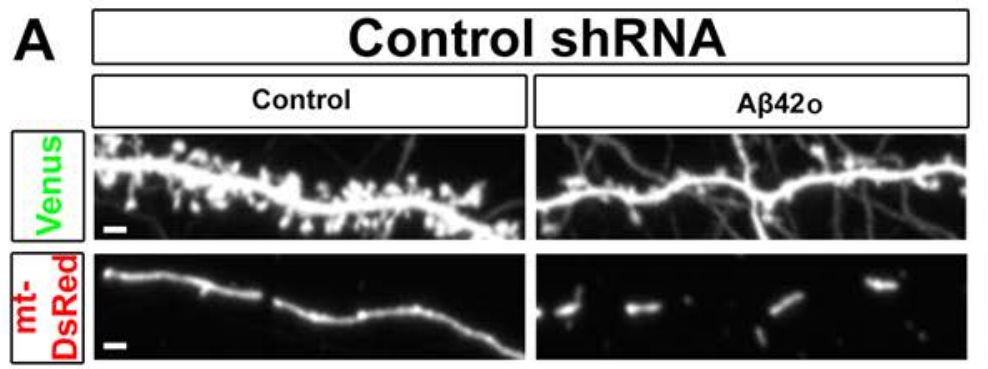

B Mitochondria Length

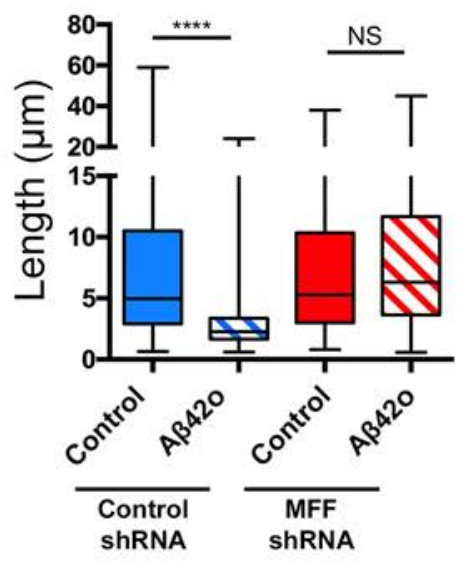

C Mitochondria Density

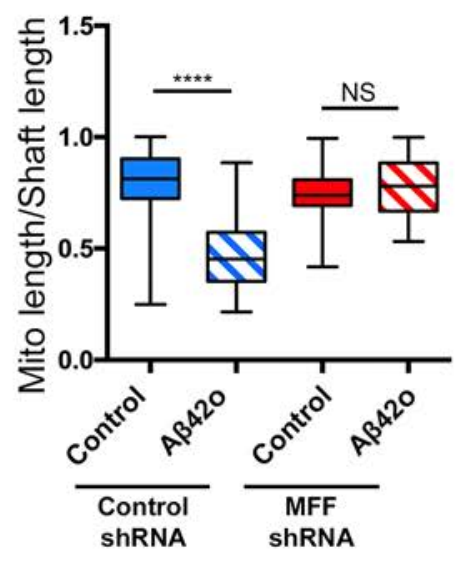

\section{MFF shRNA}

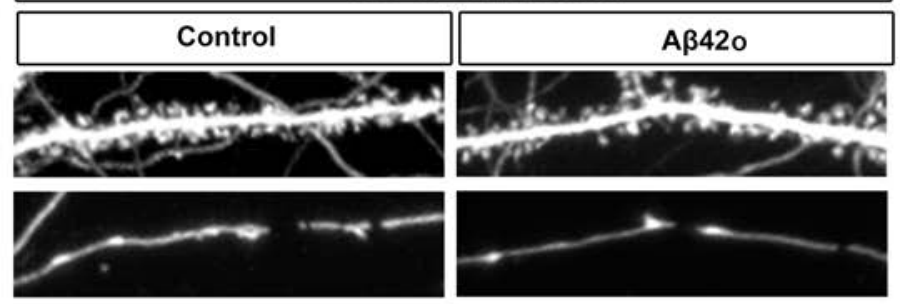

\section{Spine Density}

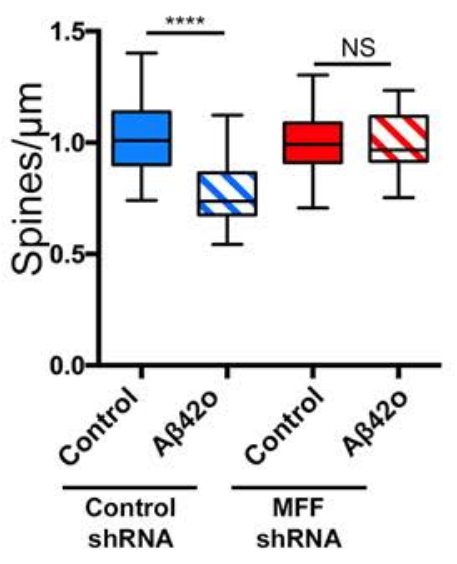

Lee, Kondapalli et al. Figure 5 


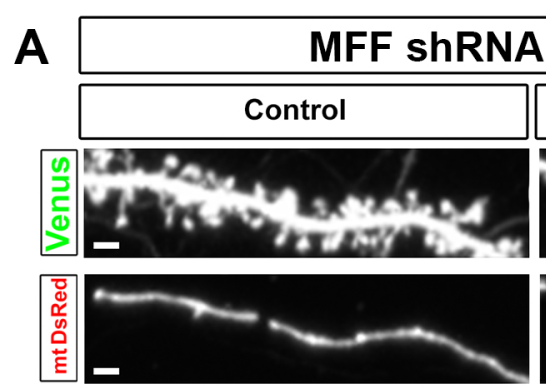

B

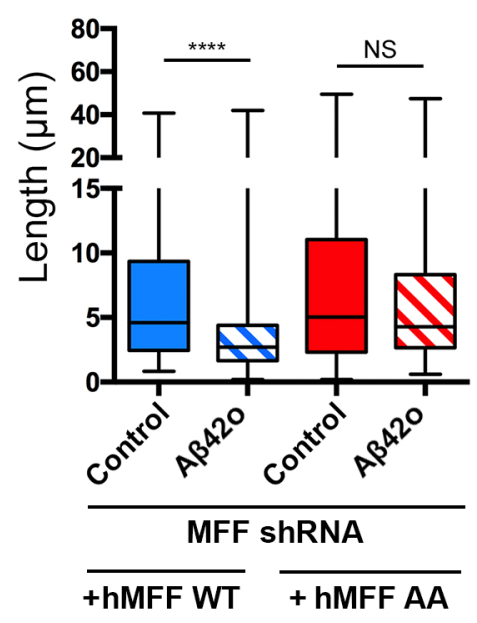

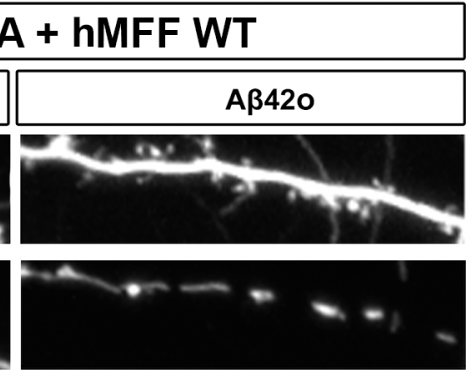

C Mitochondria Density

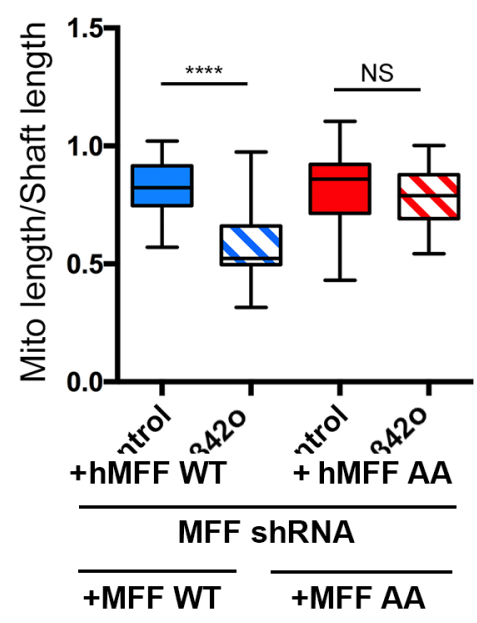

MFF shRNA + hMFF AA

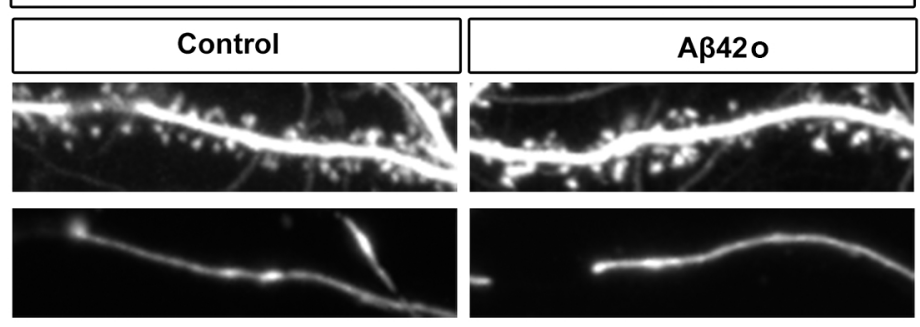

D Spine Density

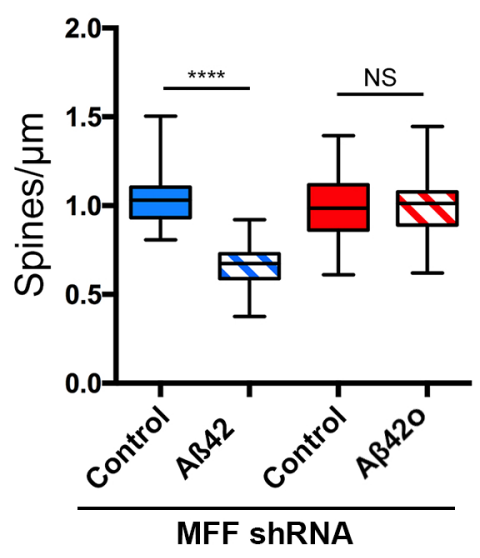

$\overline{\text { +hMFF WT }}$ 
A

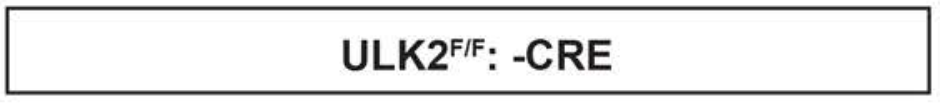

ULK2 $^{\mathrm{F} / \mathrm{F}}:+$ CRE
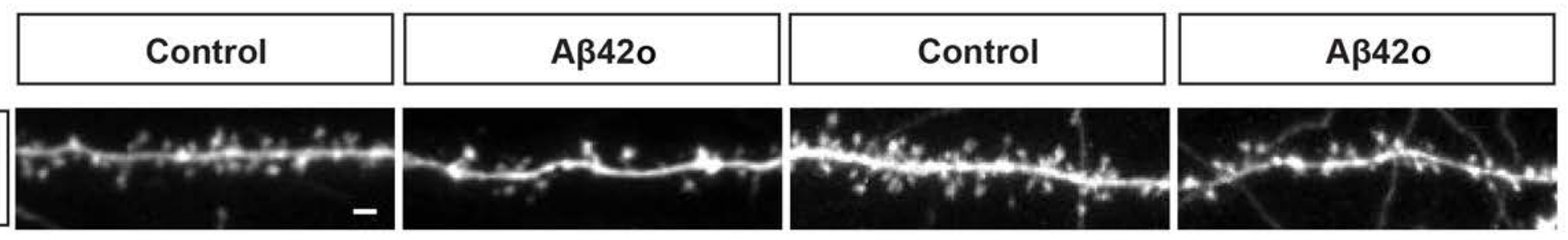

\begin{tabular}{|c|}
\hline $\begin{array}{l}\overline{0} \\
\frac{\alpha}{0} \\
\frac{0}{0}\end{array}$ \\
\hline
\end{tabular}
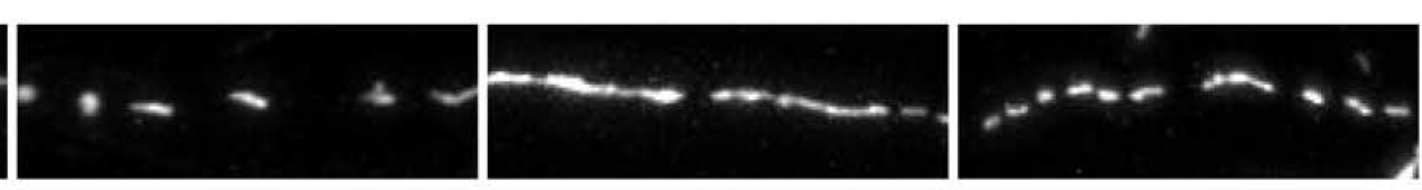

B Mitochondria length

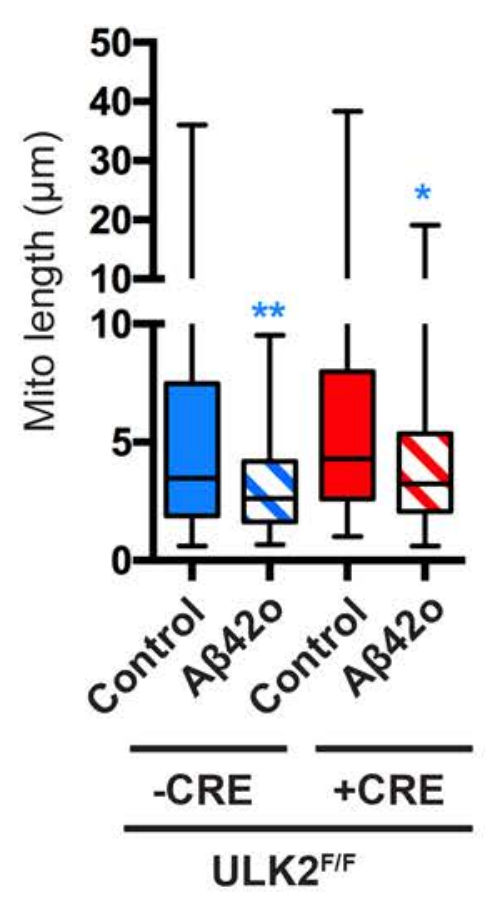

C Mitochondria density

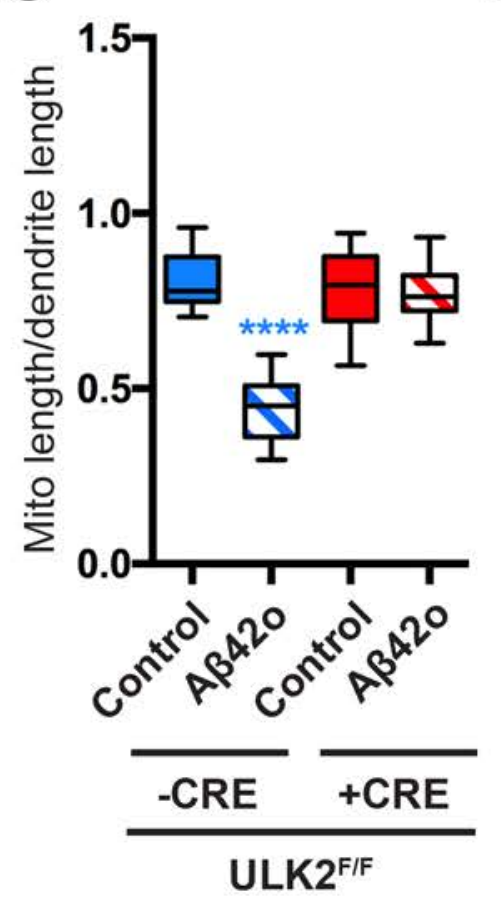

D Spine density

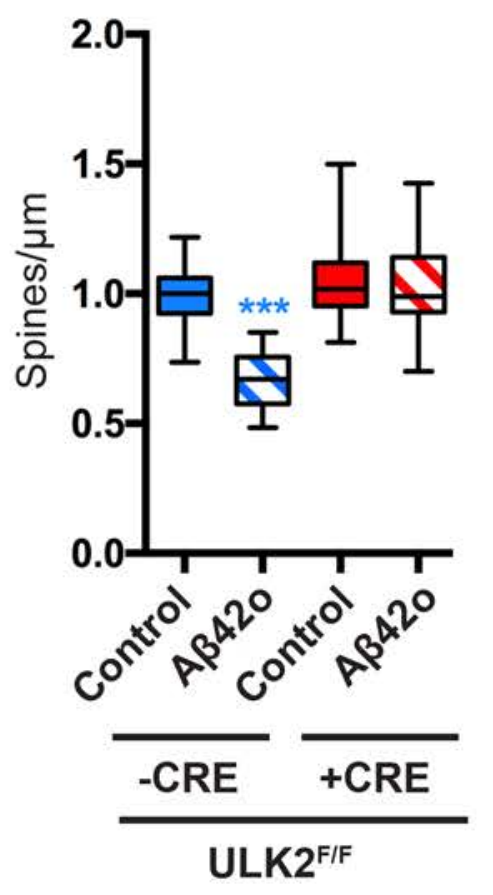

Lee, Kondapalli et a.

Figure 7 
bioRxiv preprint doi: https://doi.org/10.1101/637199; this version posted May 14, 2019. The copyright holder for this preprint (which was not certified by peer review) is the author/ffunder, who has granted bioRxiv a license to display the preprint in perpetuity. It is made available under

\begin{tabular}{|c|c|c|c|}
\multicolumn{4}{c}{ S441 S547 aCC-BY-NC-ND } \\
\\
1
\end{tabular}
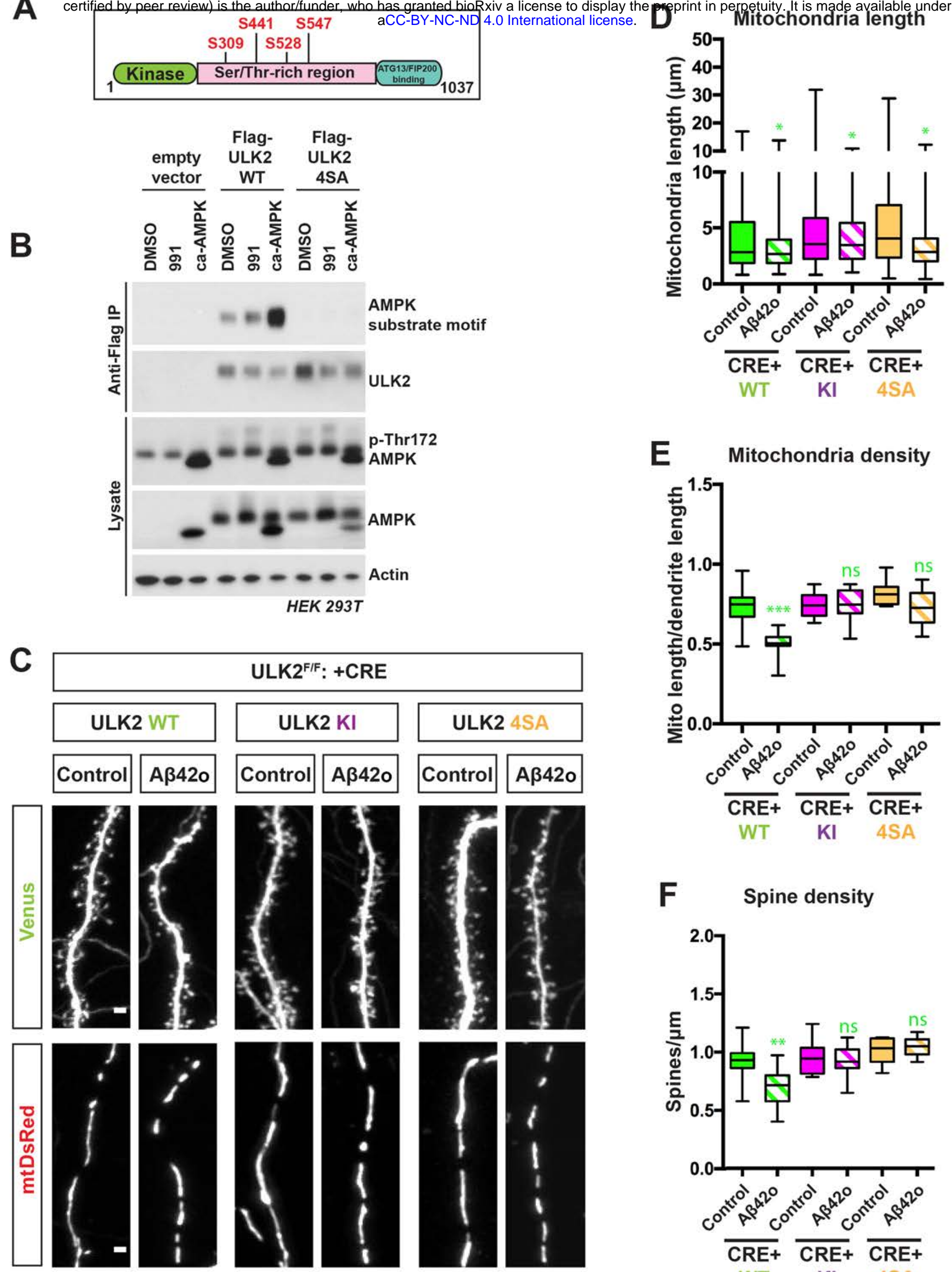

E Mitochondria density

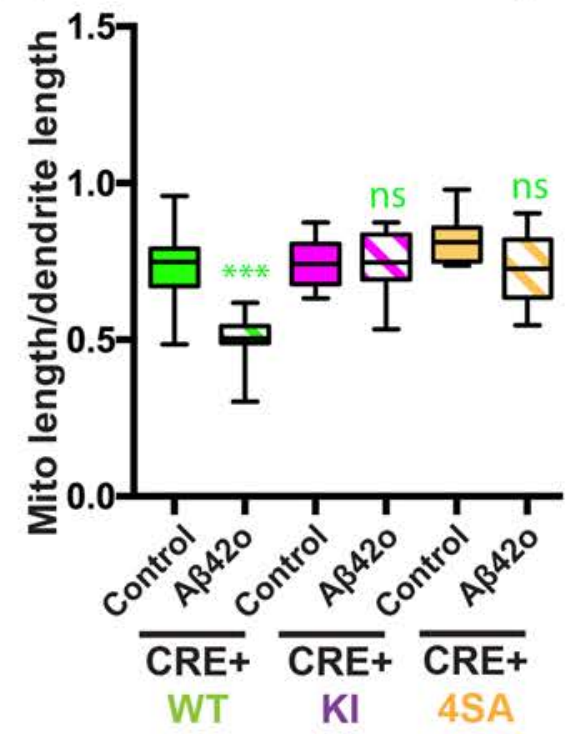

F Spine density

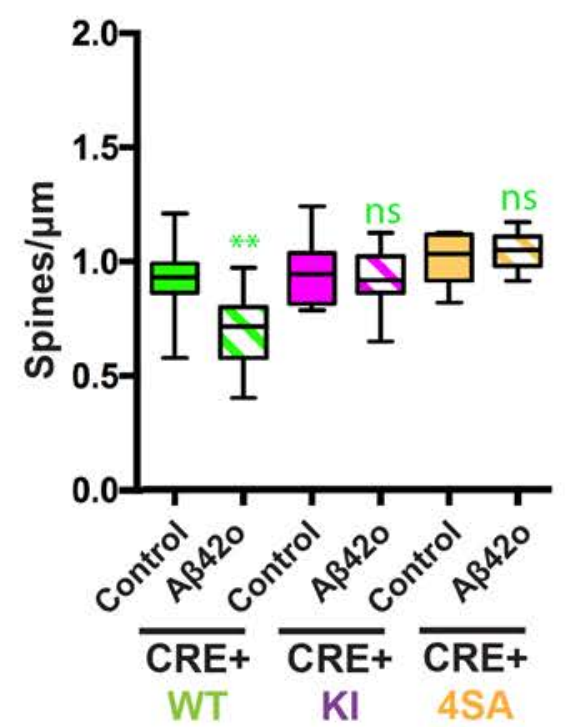

Lee, Kondapalli et al.

Figure 8 
A

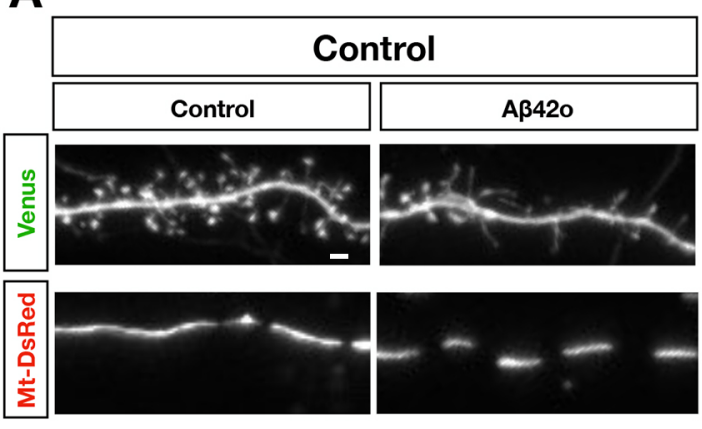

B

Mitochondria Length

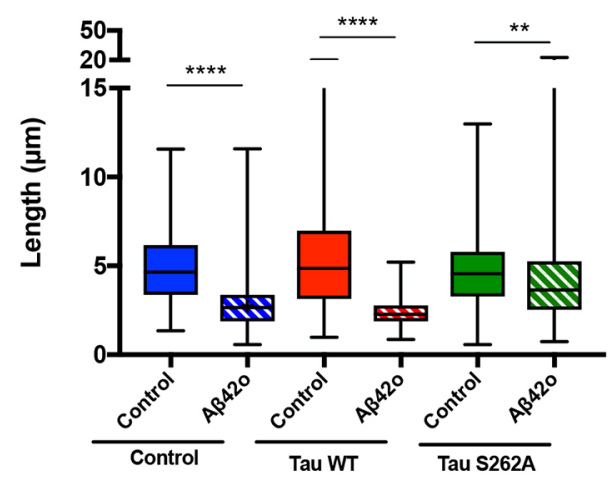

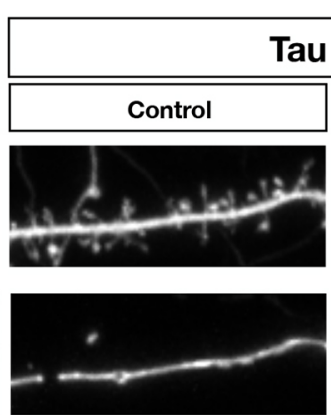

C Mitochondria Density

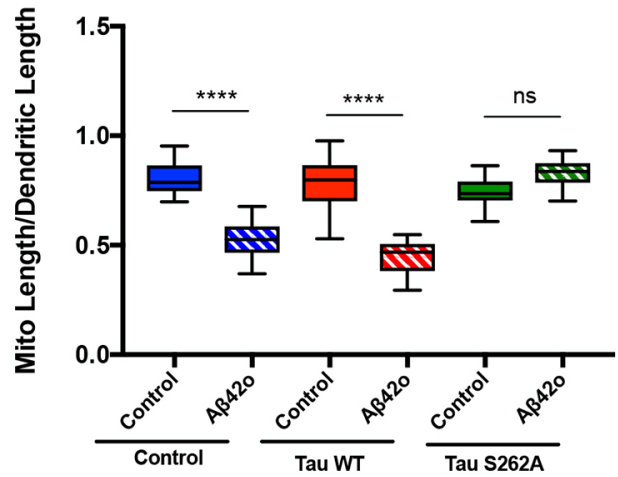

Tau S262A
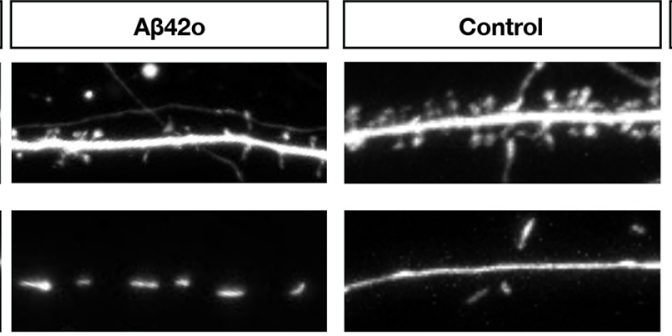

D

Spine Density

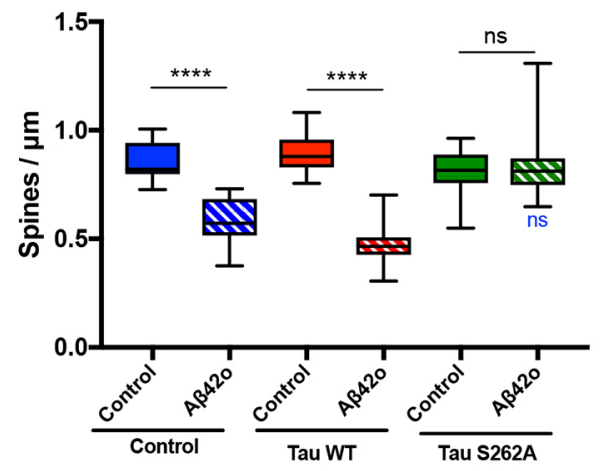

Lee, Kondapalli et al. Figure 9 
bioRxiv preprint doi: https://doi.org/10.1101/637199; this version posted May 14, 2019. The copyright holder for this preprint (which was not certified by peer review) is the author/funder, who has granted bioRxiv a license to display the preprint in perpetuity. It is made available under aCC-BY-NC-ND 4.0 International license.

\section{Supplemental Figures}

Aß42 oligomers trigger synaptic loss through

AMPK-dependent activation of mitochondrial fission and mitophagy

Lee, Kondapalli et al.

\section{SUPPLEMENTARY FIGURES 1-7}



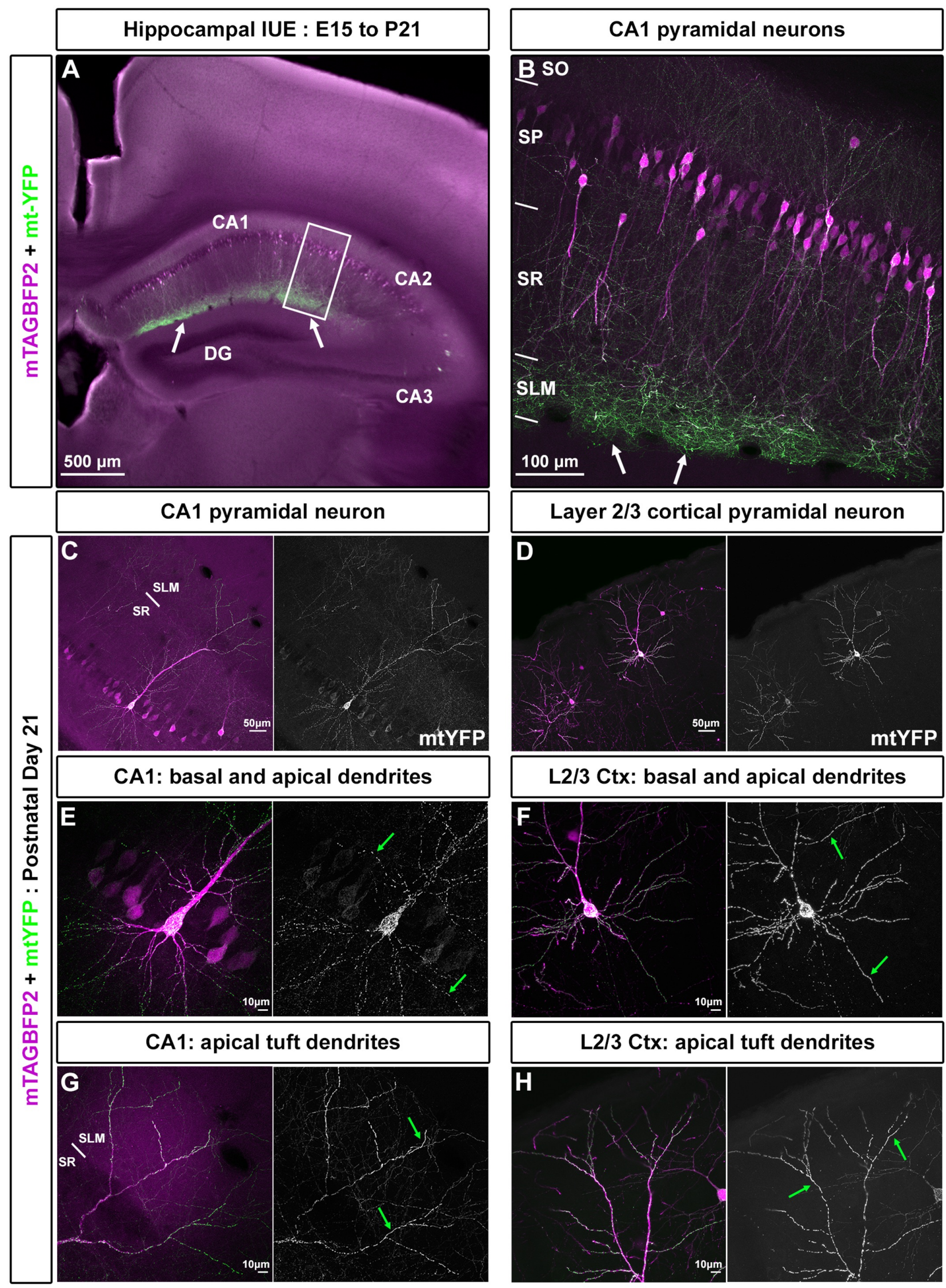

\section{Layer 2/3 cortical pyramidal neuron}

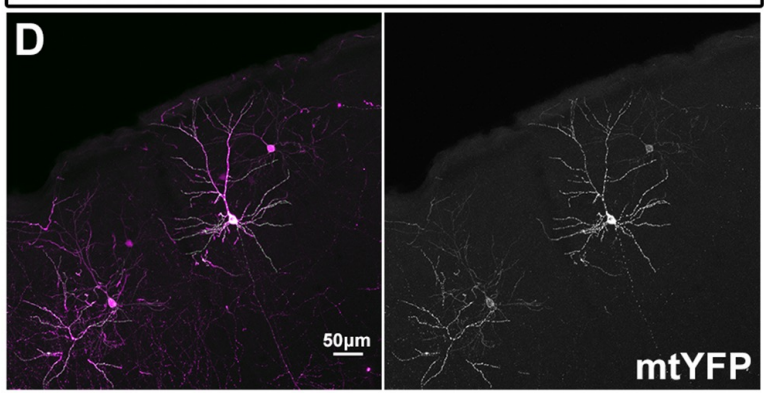

L2/3 Ctx: basal and apical dendrites
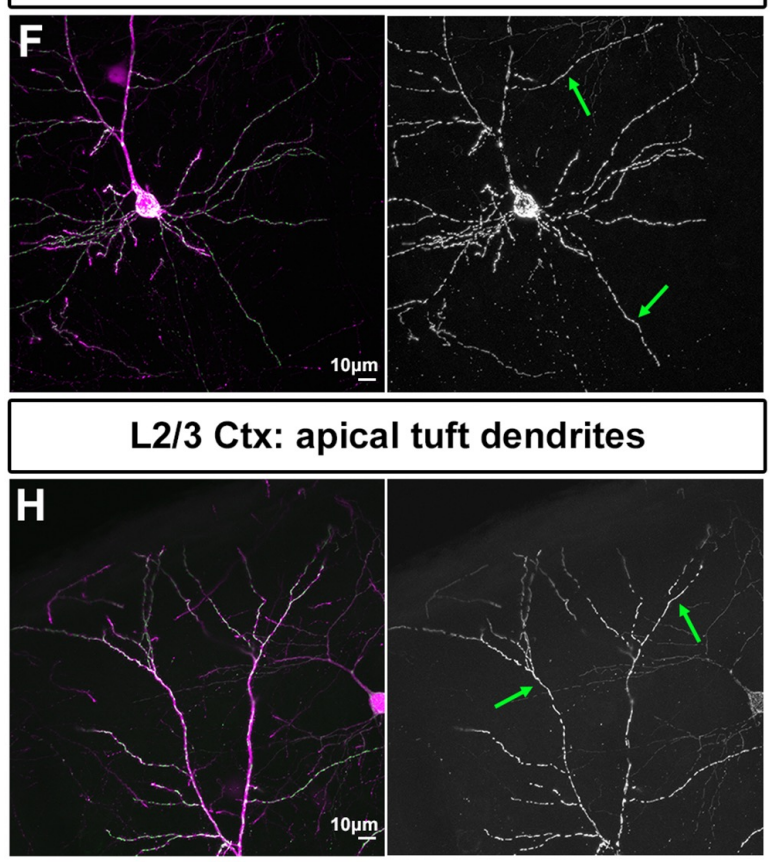
Figure S1. Hippocampal CA1 pyramidal neurons are characterized by highly compartmentalized dendritic mitochondria morphology compared to layer 2/3 cortical pyramidal neurons.

\section{Related to Figure 1}

(A) Low magnification image of hippocampal CA1 pyramidal neurons in utero electroporated at E15.5 with pCAGmTagBFP2 (purple) and pCAG-mito-YFP (green) imaged at P21. (B) High magnification image of panel A highlighting that the distal apical dendrites in the SLM layer contain high density of mitochondria compared to the basal and oblique dendrites (in so and sr, respectively). (C-D) Low magnification of a single CA1 pyramidal neuron and a single Layer 2/3 cortical pyramidal neuron. (E) High magnification of CA1 PN shown in C highlighting that mitochondria in the basal and oblique dendrites are small and fragmented (arrowheads). (F) High magnification of cortical L2/3 PN shown in D, highlighting that mitochondria morphology is homogenously elongated (arrow) in basal and proximal apical dendrites. (G) High magnification of CA1 PN shown in C highlighting that mitochondria morphology in the distal apical tuft dendrites (s/m) are elongated unlike those found in basal and oblique dendrites of the same cell (see E). (H) High magnification of cortical L2/3 PN highlighting that mitochondria morphology is homogenously elongated in distal apical dendrites similar to those found in both basal and oblique dendrites in the same neuron (see F). Abbreviations: slm, stratum lacunosum moleculare; sr, stratum radiatum; so, stratum oriens; sp, stratum pyramidalis. 

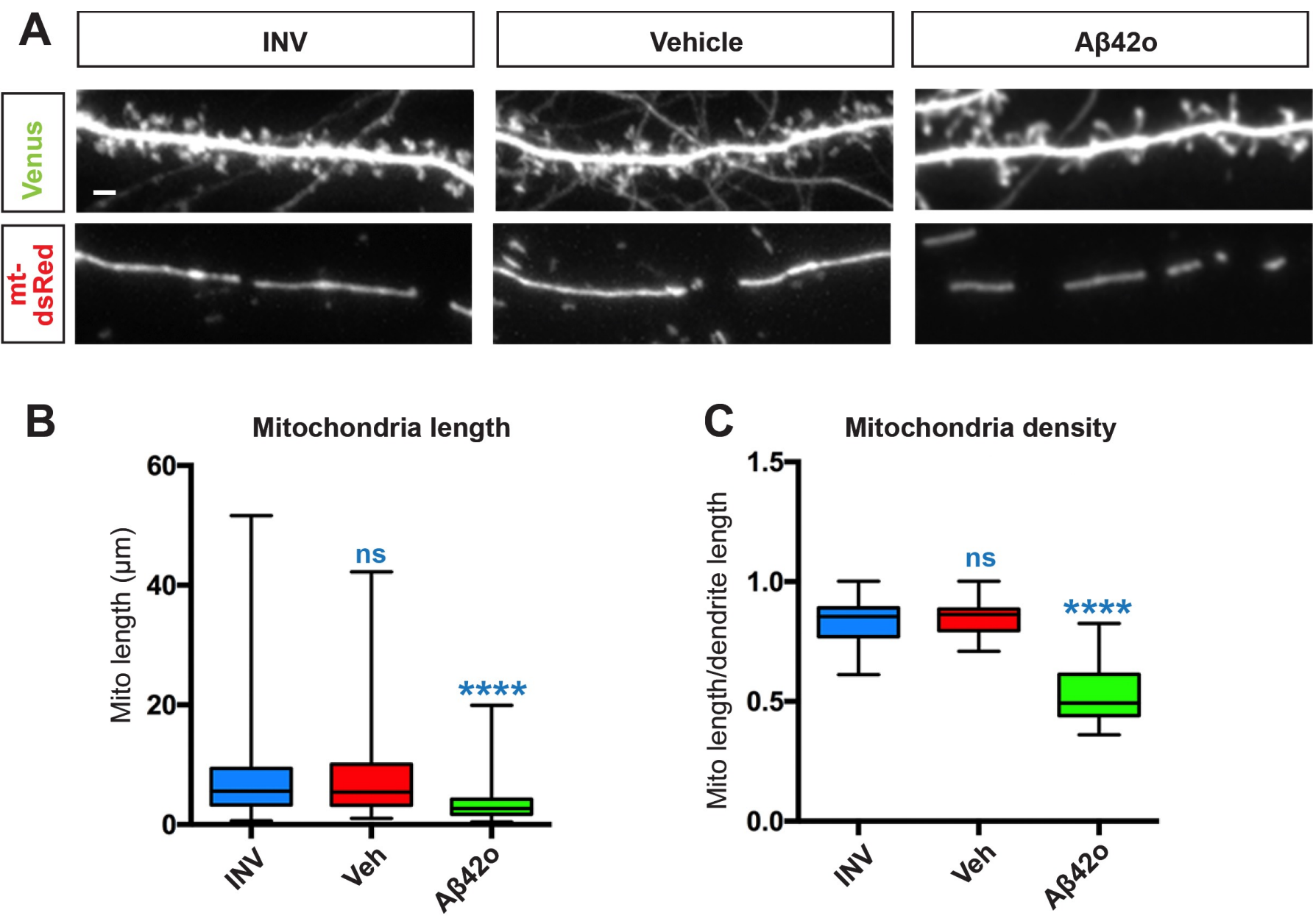

$\mathbf{D}$
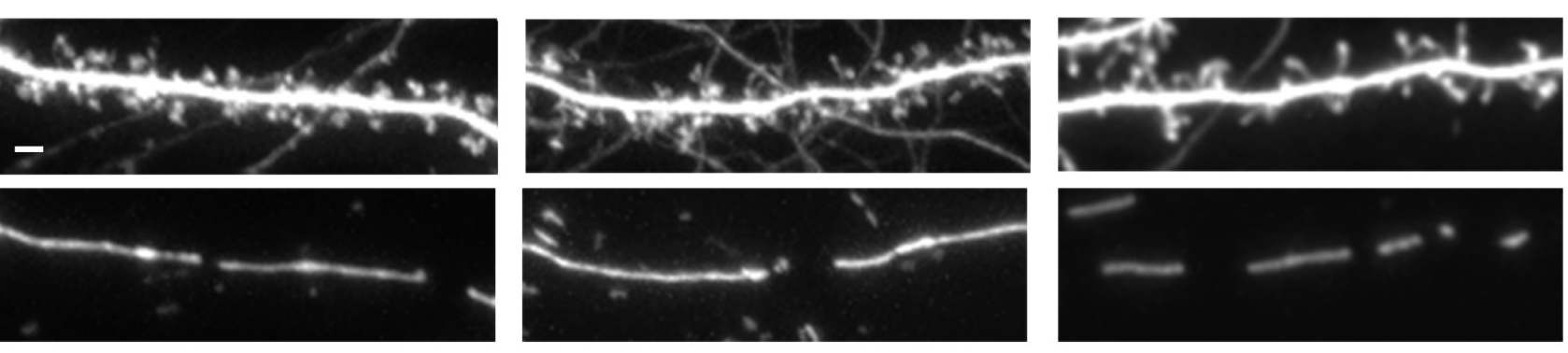

B

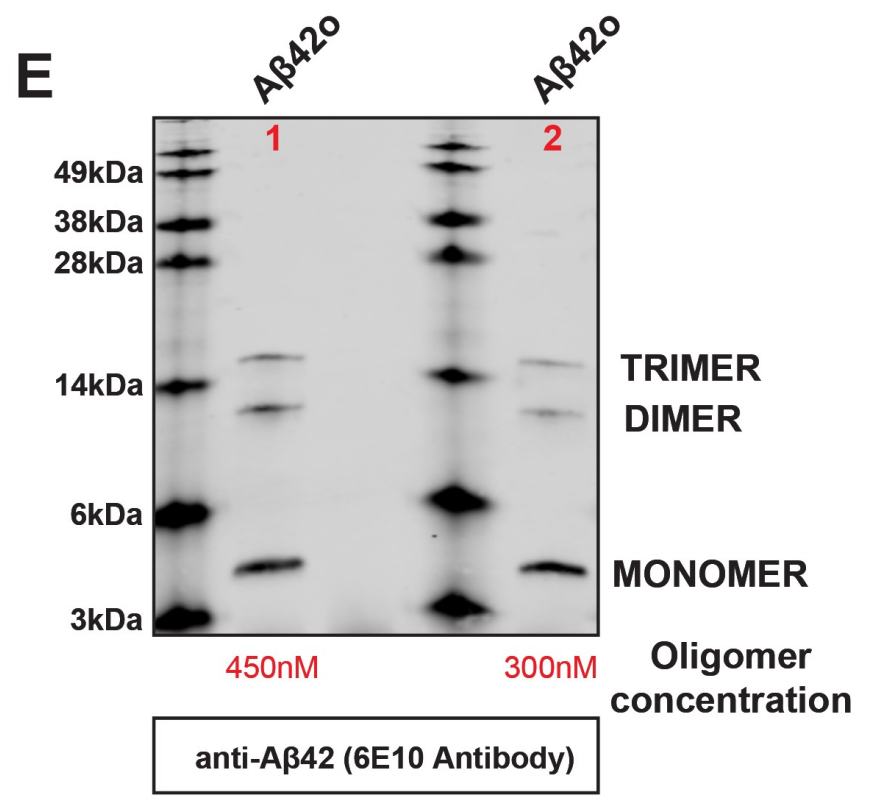

Figure S2. Treatment of inverse oligomeric A 342 does not influence mitochondrial morphology or spine density. 


\section{Related to Figure 2}

(A) Representative high magnification images of dendritic segments showing dendritic spines in the upper panel and mitochondria in the lower panel. Treatment of inverse $A \beta 420$ does not affect spine density or mitochondrial morphology and have similar parameters as DMSO treated neurons (B) Mitochondrial length for inverse A 342 is comparable to DMSO treated neurons while $A \beta 42$ oligomer treatment significantly reduces mitochondrial length. (C) Mitochondrial density for inverse $A \beta 42$ is comparable to DMSO treated neurons while A 342 oligomer treatment significantly reduces mitochondrial density. (D) Spine density quantification for inverse $A \beta 42$ is comparable to DMSO treated neurons while A 442 oligomer treatment significantly reduces spine density. All the analysis was done blind to the experimental conditions, and was done by manual counting using FIJI. Data is represented by box plots displaying minimum to maximum values, with the box denoting $25^{\text {th }}, 50^{\text {th }}$ (median) and $75^{\text {th }}$ percentile from 3 independent experiments. $\mathrm{n}_{\mathrm{INV}}=41$ dendrites, 243 mitochondria; $\mathrm{n}_{\mathrm{veh}}=31$ dendrites; 178 mitochondria; $n_{A \beta 420}=33$ dendrites, 245 mitochondria. Statistical analysis was performed using Kruskal-Wallis test followed by Dunn's post-hoc test in (B-D). The test was considered significant when $p<0.05$ with the following criteria: * $p<0.05 ;{ }^{* *} p<0.01 ;{ }^{* * *} p<0.001 ;{ }^{* * *} p<0.0001$; ns, not significant. Scale bar $=2 \mu \mathrm{m}$. (E) $16.5 \%$ Tris-Tricine SDS-PAGE was performed to resolve $A \beta 42$ oligomers from the peptide monomer that were generated as described in Material and Methods. Immuno-blotting was performed with 6E10 antibody which shows a mixture of monomers, dimers and trimers in lane 1 and 2 (two independent oligomerization experiments). For quantifying the relative oligomer/monomer concentration in lane 1 and 2, Near-infrared fluorescence signal intensity was measured for dimer+trimer and monomer using an Odyssey Imager. Signal intensity values for Lane1: Dimer+Trimer-1510; monomer-1830. Signal intensity values for Lane2: Dimer+Trimer-736; monomer-1720. 
A
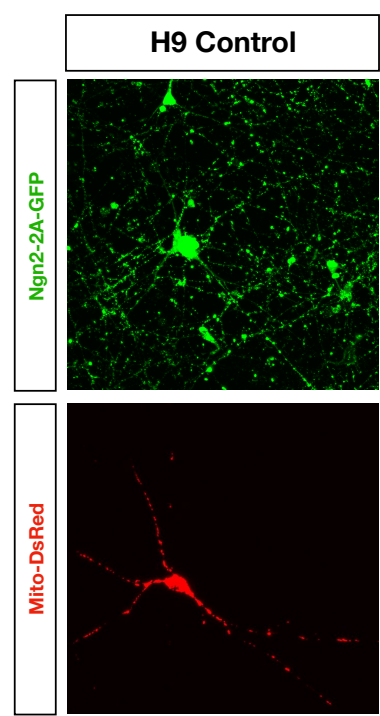

$\infty+\infty \ldots+\ldots$
B
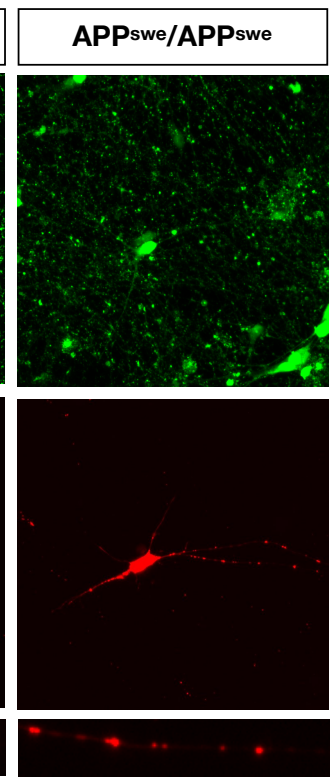

D
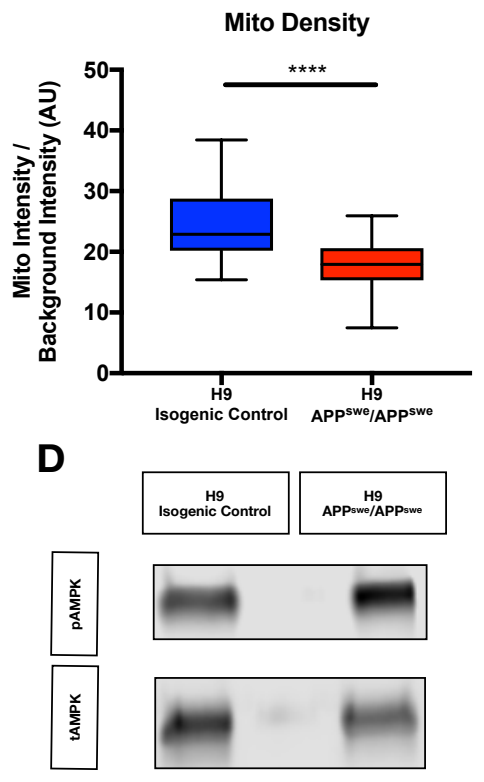

C

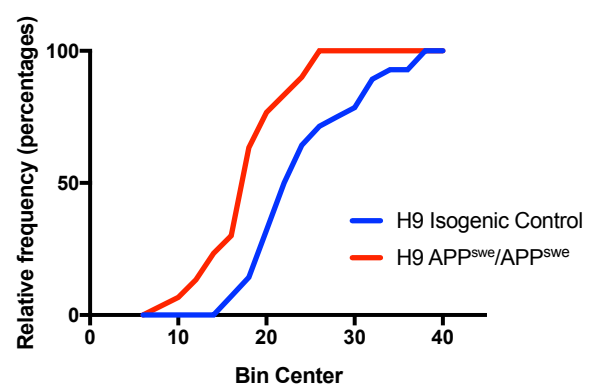

E

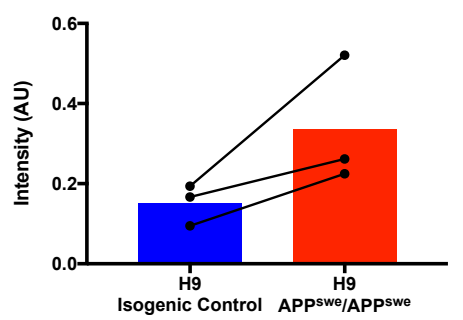

Figure S3. hESC-derived cortical neurons expressing endogenous APP ${ }^{\text {SWE }}$ mutations exhibit decreases in mitochondrial density and increased AMPK activation relative to isogenic controls.

(A) Representative images neurons of control H9 embryonic stem (hESC) cells and H9: APPSWE/SWE knockin hESCs at 24 DIV (post dox-induction). hESC-derived cortical-like neurons were induced using Ngn2-2A-eGFP driven neural induction (see methods), which allows visualization by eGFP. Neurons were transfected using Lipofectamine 3000 at 21-31 DIV with a pCAG-mitoDSRed reporter gene to label mitochondria, fixed 4 days post transfection, and imaged by confocal microscopy. Endogenous expression of the APPSWE/SWE mutation is sufficient to induce a significant decrease in mitochondrial density in their dendrites (bottom high magnification panels) relative to isogenic control neurons. (B) Quantification of neuritic mitochondrial density. All of the analyses were done by kymographic fluorescent density measurement in Nikon Elements software. (C) Cumulative frequency distribution of mitochondrial density in Ngn2-induced cortical neurons derived from control H9 (blue) and APP ${ }^{\text {SWEISWE }}$ cells (red). (D) Sample western blot of H9 isogenic control vs. H9: APP ${ }^{\text {SWEISWE }}{ }^{\text {cortical }}$

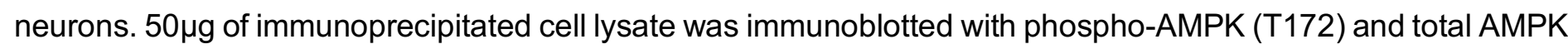
to confirm activation of AMPK under endogenous expression of APPSWE mutation. (E) Quantification of increased phospho-AMPK, representing mean and SEM. All of the statistical analyses were performed in Prism 6 (GraphPad Software). Data is represented by box plots displaying minimum to maximum values, with the box denoting 25th, 50th (median) and 75th percentile from 3 independent experiments. In A-C: $\mathrm{n}_{\text {H9 control }}=227$ neurites, 70 neurons; $n_{A P P s w e}$ control $=203$ neurites, 73 neurons. Statistical analyses were performed using One- 
bioRxiv preprint doi: https://doi.org/10.1101/637199; this version posted May 14, 2019. The copyright holder for this preprint (which was not certified by peer review) is the author/funder, who has granted bioRxiv a license to display the preprint in perpetuity. It is made available under aCC-BY-NC-ND 4.0 International license.

way ANOVA followed by Kruskal-Wallis post-hoc test in B. The test was considered significant when $p<0.05$ with the following criteria: ${ }^{* * * *} p<0.0001$; ns, not significant. 

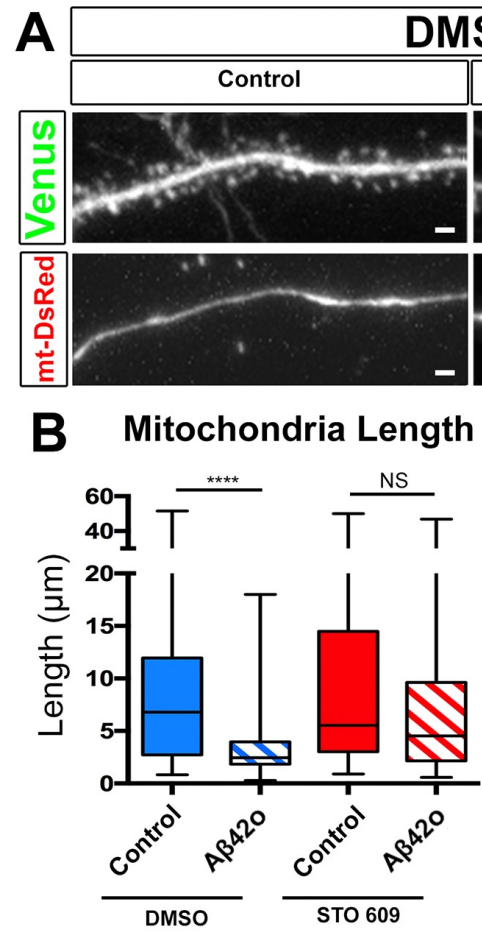
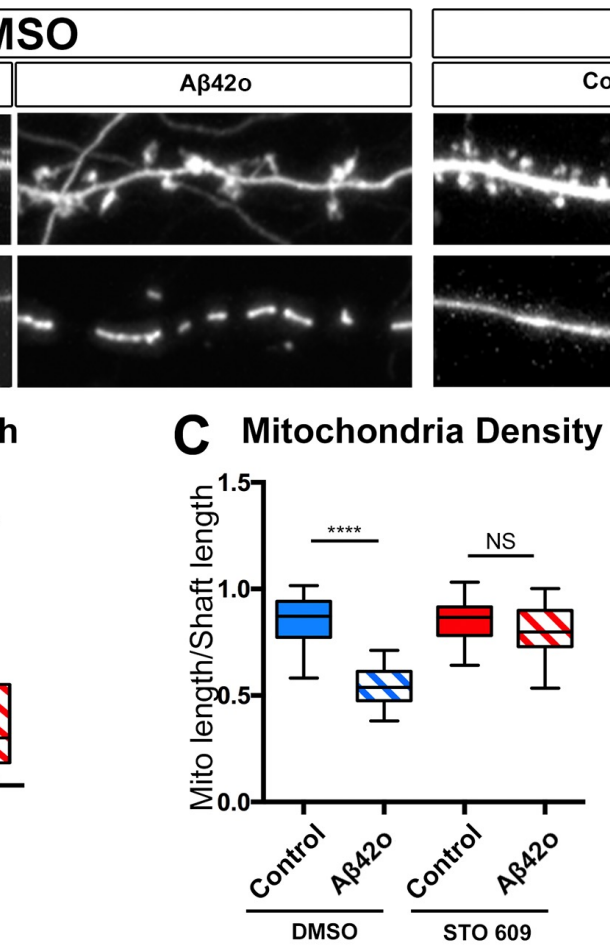
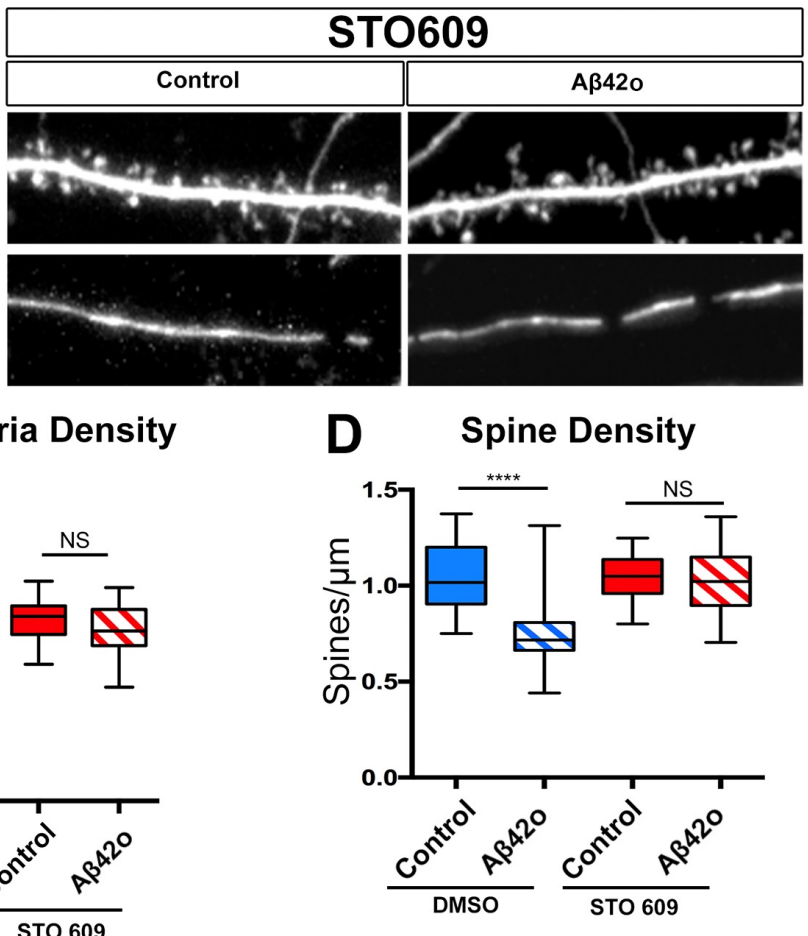

Figure S4. Oligomeric Aß42 induced synaptotoxicity and dendritic mitochondrial fragmentation is CAMKK2 dependent.

\section{Related to Figure 4}

(A) Dendritic segments (secondary) of primary cortical neuron at 21 DIV. Embryos at E15.5 were ex utero electroporated with pCAG-Venus and pCAG-mito-DsRed. At 20 DIV, neurons were pre-treated with either DMSO or STO609 $(2.5 \mu \mathrm{M})$ for 2 hours before being treated with either control or A 342 oligomers for 24 hr. Blocking CAMKK2 activity by STO609 blocks both dendritic mitochondrial remodeling and synaptotoxicity. (B) Quantification of dendritic mitochondrial length, (C) dendritic mitochondrial density, and (D) spine Density. All the analysis was done blind to the experimental conditions, and was done by manual counting using FIJI. Data is represented by box plots displaying minimum to maximum values, with the box denoting $25^{\text {th }}, 50^{\text {th }}$ (median) and $75^{\text {th }}$ percentile from 3 independent experiments. $\mathrm{n}_{\mathrm{DMSO}}$ control $=37$ dendrites, 163 mitochondria; $\mathrm{n}_{D M S O A \beta 420}=$ 31 dendrites; 248 mitochondria; $\mathrm{n}_{\mathrm{STO}} 609$ control $=37$ dendrites; 163 mitochondria; $\mathrm{n}_{\mathrm{STO} 609 \text { A } 420}=35$ dendrites, 157 mitochondria. All of the statistical analyses were performed with Prism 6 (GraphPad Software). Statistical analysis was performed using Kruskal-Wallis test followed by Dunn's post-hoc test in (B-D). The test was considered significant when $p<0.05$ with the following criteria: ${ }^{*} p<0.05 ;{ }^{* *} p<0.01 ;{ }^{* *} p<0.001 ;{ }^{* * *} p<0.0001$; ns, not significant. Scale bar $=2 \mu \mathrm{m}$. 


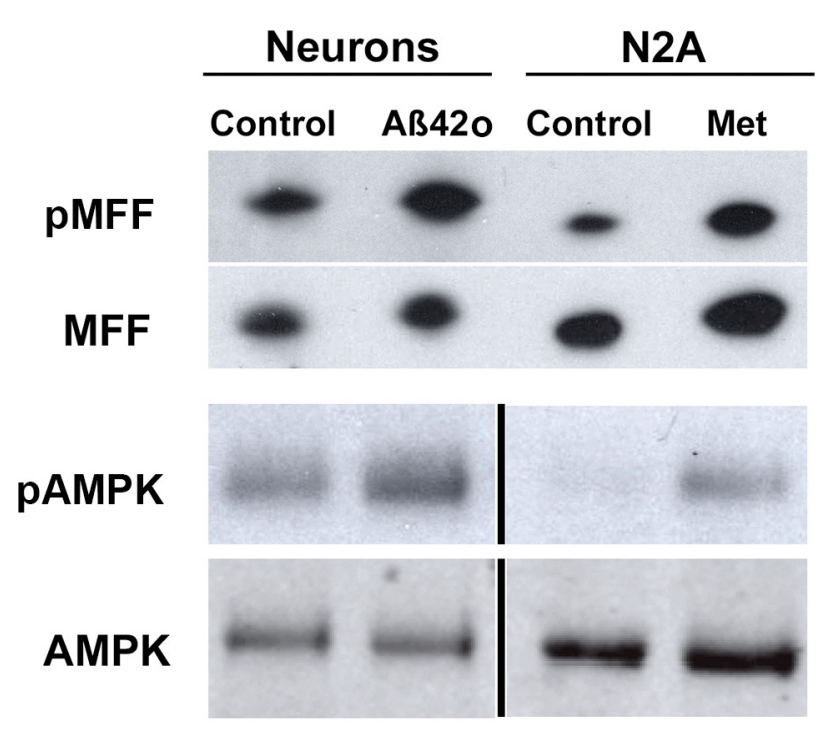

Figure S5. Aß420-dependent increase in MFF phosphorylation.

\section{Related to Figure 6}

Cortical PNs maintained in dissociated culture were treated at 20DIV with either control vehicle or A 3420 for 24 hours. N2A cells were treated with either DMSO or $2 \mathrm{mM}$ of Metformin for 5 hours as positive control for AMPKdependent MFF phosphorylation (Toyama et al., 2016). 80 $\mu \mathrm{g}$ of whole cell lysate was immunoprecipitated with MFF antibody (Progen) and immunoblotted with MFF antibody or phospho-MFF antibody (S146, CST). Whole cell lysates were also immunoblotted with phospho-AMPK (T172, CST) and AMPK to confirm activation of AMPK under both $A \beta 420$ for 24 hours (Mairet-Coello et al., 2013) and metformin treatment (Toyama et al., 2016). 
A
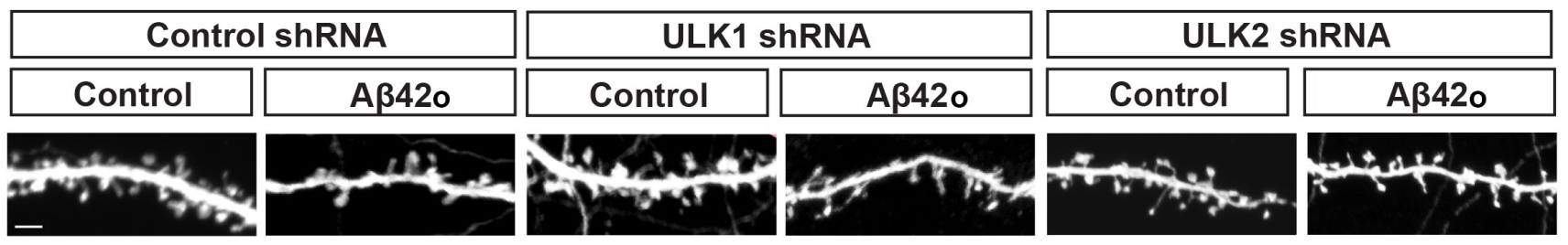

B

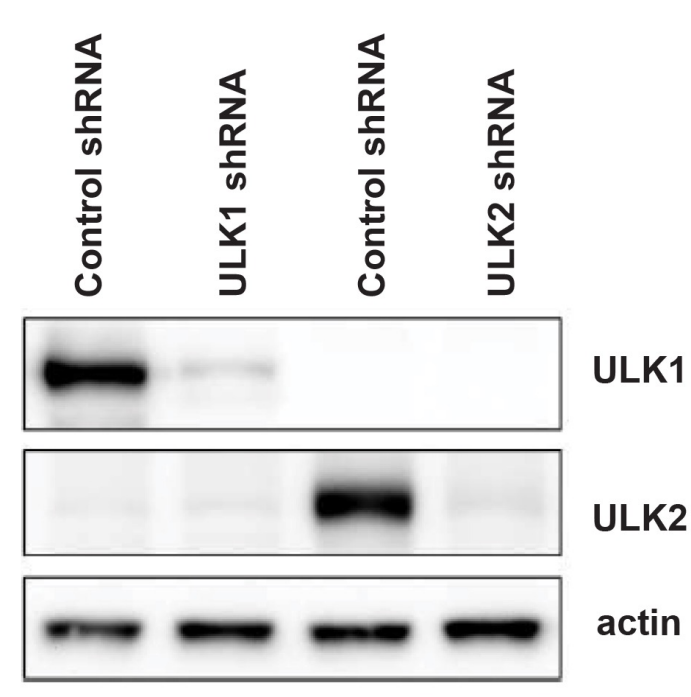

HEK293T
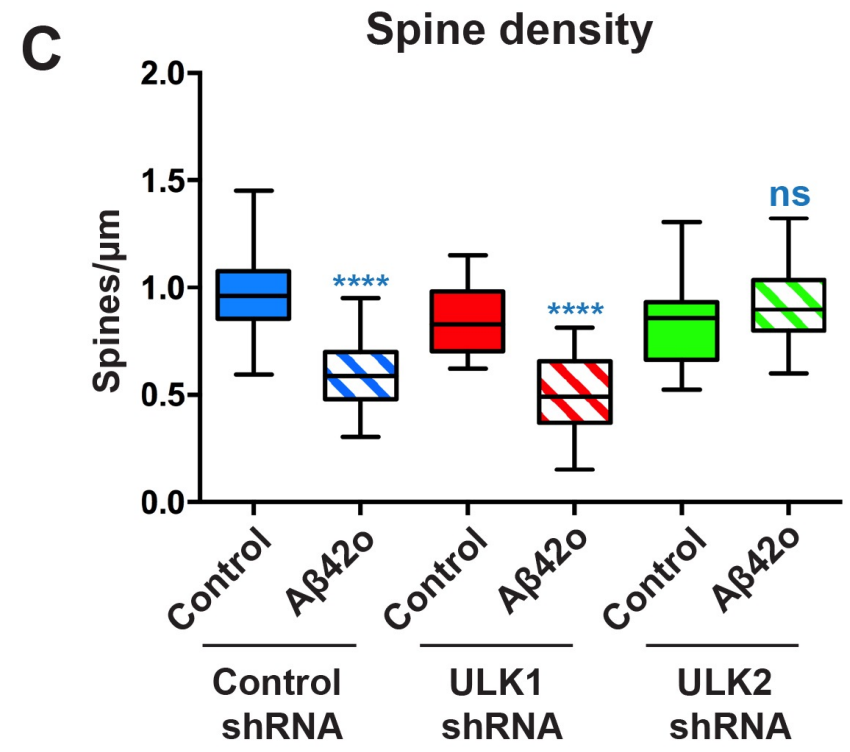

Figure S6. ULK2 but not ULK1 lies downstream is required for Aß420-dependent synaptotoxic effects.

\section{Related to Figure 7}

(A) Representative images of secondary dendritic segments of primary cortical neurons treated with either control or $A \beta 420$ at 21DIV for 24 hours. Embryos were subjected to ex-utero electroporation at E15.5 with pCAGVenus and control shRNA or an shRNA specific for the kinase ULK1 or ULK2. Knockdown of ULK2, but not ULK1 kinase blocks oligomeric Aß42o-induced synaptotoxicity. (B) Western blot showing validation of ULK1 and ULK2 shRNA and the specificity of the shRNAs for the respective isoform. HEK 293T cells were transiently cotransfected with control shRNA or ULK1/ULK2 shRNA along with over-expressed myc-mULK1 and Flag-mULK2 respectively and western blotting was performed with $25 \mu \mathrm{g}$ of lysate with the indicated antibody. (C) Quantification of spine density for panel A. All the analysis was done blind to the experimental conditions, and was done by manual counting using FIJI. All of the statistical analyses were performed with Prism 6 (GraphPad Software). Data is represented by box plots displaying minimum to maximum values, with the box denoting $25^{\text {th }}$, $50^{\text {th }}$ (median) and $75^{\text {th }}$ percentile from 3 independent experiments. $n_{P L K O}$ control $=31$ dendrites; $n_{P L K O ~ A \beta 420}=27$

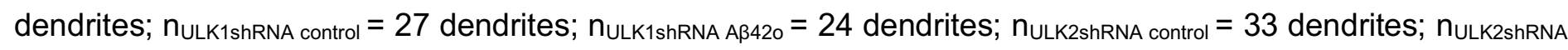
$\mathrm{A}_{420}=35$ dendrites. Statistical analysis was performed using Kruskal-Wallis test followed by Dunn's post-hoc test in (C). The test was considered significant when $p<0.05$ with the following criteria: ${ }^{*} p<0.05$; ${ }^{* *} p<0.01$; ${ }^{* * *} p<0.001 ;{ }^{* * * *} p<0.0001 ; n s$, not significant. Scale bar $=2 \mu \mathrm{m}$. 
bioRxiv preprint doi: https://doi.org/10.1101/637199; this version posted May 14, 2019. The copyright holder for this preprint (which was not certified by peer review) is the author/funder, who has granted bioRxiv a license to display the preprint in perpetuity. It is made available under aCC-BY-NC-ND 4.0 International license. 
A

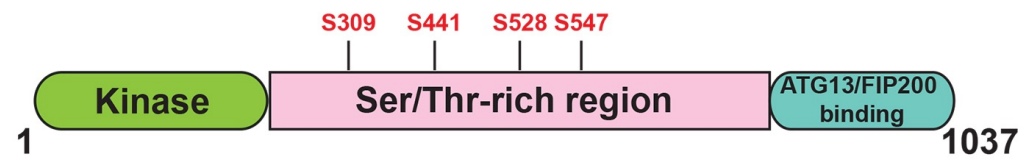

B
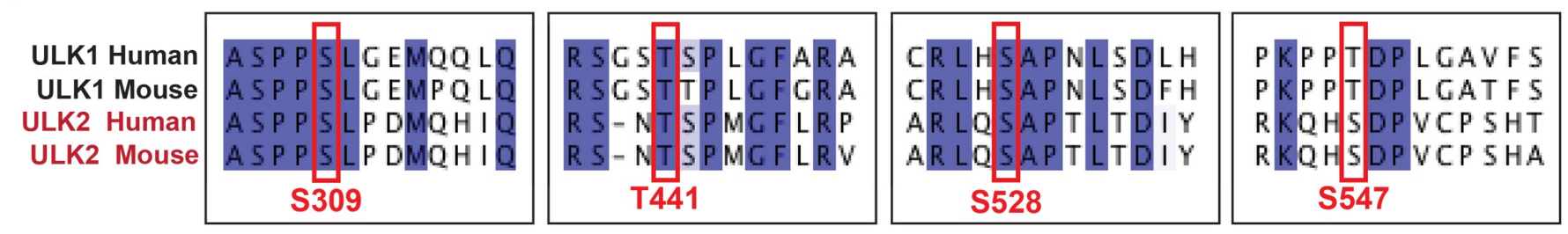

Figure S7. Schematic of conserved AMPK-mediated phosphorylation sites in ULK2.

\section{Related to Figure 8}

Schematic of mULK2 domain structure highlighting the four predicted AMPK-mediated phosphorylation sites. (B) ClustalW multiple sequence alignment of human and mouse ULK1 and ULK2 showing a high degree of conservation of four AMPK-mediated phosphorylation sites in ULK2 which were previously reported in ULK1 (Toyama et al., 2016). 
Supplementary Movie S1 Time-lapse of DIV 21 Inverse peptide treated cortical pyramidal neurons expressing mito-mTagBFP2, LAMP1-mEmerald and mRFP-LC3 to visualize mitochondria, lysosome and autophagosome (dendritic segment corresponds to the representative image in Fig. 3). Neurons were imaged every 15min for 14hr. Related to Figure 3.

Supplementary Movie S2 Time-lapse of DIV 21 A 3420 treated cortical pyramidal neurons expressing mitomTagBFP2, LAMP1-mEmerald and RFP-LC3 to visualize mitochondria, lysosome and autophagosome (dendritic segment corresponds to the representative image in Fig. 3). Neurons were imaged every 15min for 14hr. Related to Figure 3. 


\section{Supplementary Experimental Procedures}

\section{Aß42 oligomers trigger synaptic loss through \\ AMPK-dependent activation of mitochondrial fission and mitophagy}

Lee, Kondapalli et al.

\section{Constructs}

pSCV2-Venus and pCAG-mito-dsRed were described in previous publication (Lewis et al., 2016). pLKO-MFFsh specific for mouse was obtained from Sigma Aldrich (TRCN0000174665). Validation of the shRNA knockdown efficiency was done elsewhere (Lewis et al., 2018). cDNA encoding Renalin, MFF WT (Uniprot Q9GZY8 isoform 5), and MFF AA were described previously (Toyama et al., 2016) and sublconed in the pCAG plasmid backbone 3 ' to CAG promoter using PCR. $0.25 \mu \mathrm{g} / \mathrm{mL}$ of individual constructs were used for the MFF rescue experiments. pCAG-Cre, pCAG-Scrambled Cre, pCIG-hTau (isoform 4R2N), and non-phosphorylatable hTau-S262A were described in previous publications (Courchet et al., 2013). pcDNA3-flag-ULK2 specific for mouse was obtained from Addgene (\#27637) and was used to generate the kinase-inactive (K39I) and serine-to-alanine 4SA mutant (S309/T441/S528/S547A) using Quikchange II site directed mutagenesis kit (Agilent technologies) and were subcloned into pCAG vector backbone using PCR. pSUPER-scrambled, ULK1 shRNA and ULK2 shRNA constructs were validated in a previous study (Zhou et al., 2007).

\section{In utero hippocampal electroporation}

Timed pregnant hybrid F1 females were obtained by mating inbred 129/SvJ females and C57BI/6J males. These F1 females were then mated with J20 males to obtain both mice that do express the APP transgene and mice that do not express the transgene in the same littermate. In utero electroporations were performed as detailed in (Szczurkowska et al., 2016) with the exception that J20 mice were used. Briefly, a mix of endotoxin-free plasmid preparation (PSCV2-mVenus $-1 \mu \mathrm{g} / \mathrm{mL}$, pCAG-mitoDsRed $-0.5 \mu \mathrm{g} / \mathrm{mL}$ ) was injected into both lateral hemisphere of E15.5 embryos using a picospritzer. Electroporation was performed with triple electrode to target hippocampal progenitors in E15.5 embryos by placing the two anodes (positively charged) on either side of the head and the cathode on top of the head at a $0^{\circ}$ angle to the horizontal plane. Four pulses of $45 \mathrm{~V}$ for $50 \mathrm{~ms}$ with $500 \mathrm{~ms}$ interval were used for the electroporation.

\section{Tissue slice preparation and Immunofluorescence}

Animals were sacrificed 3 months after birth by terminal transcardial perfusion of $4 \%$ paraformaldehyde (PFA, Electron Microscopy Sciences) followed by overnight post-fixation in 4\% PFA. Brains were washed 3X with PBS for 5 minutes and sectioned at 100 $\mu$ m thickness using vibratome (Leica). Sections were permeabilized with $0.2 \%$ Triton X-100 3X for 5 minutes, blocked in PBS based blocking buffer with $5 \%$ BSA and $0.2 \%$ Triton, and stained with chicken anti-GFP (Aves, 1:1000) and rabbit anti-RFP (Abcam, 1:1000) for enhancement of PSCV2-mVenus and pCAG-mitoDsRed, respectively overnight at $4^{\circ} \mathrm{C}$. Sections were washed with $0.2 \%$ Triton buffer $3 \mathrm{X}$ for 5 minutes and incubated with Alexa488- and Alexa 555- labeled secondary antibodies for PSCV2-mVenus and pCAG-mitoDsRed, respectively overnight at $4^{\circ} \mathrm{C}$. Sections were washed with $0.2 \%$ Triton buffer $3 \mathrm{X}$ for 10 minutes each and mounted using VectaShield $®$ Mounting Medium (Vector Laboratory).

\section{Drug Treatments}

Neuronal cells at 20-25 DIV were treated with STO609 $(2.5 \mu \mathrm{M}$, Millipore), a CAMKK2 inhibitor, 2.5 hours prior to A 342 treatment. N2A cells were transfected with pCAG-MFF-WT construct using the recommended protocol from FuGENE®HD Transfection Reagent overnight. Cells were treated with either DMSO or Metformin (2mM, Sigma) for 5 hours before cells were lysed using Triton lysis buffer as described under Cell Lysis and Immunoprecipitation. 


\section{eSC Immunoprecipitation and Western Blotting}

For phospho-T172 AMPK and total AMPK for eSC experiments, equal amounts of lysates $(50 \mu g)$ were immunoprecipitated with phospho-T172 AMPKa or total AMPKa to enhance AMPK proteins. Lysates from H9Control and H9APP lines were incubated overnight at $4^{\circ} \mathrm{C}$ in a rotator with either phospho-T172 AMPK or total AMPK primary antibodies. Protein A-Agarose beads (Sigma) were washed three times with PBS and added to the immuno-bound lysates for at least $1 \mathrm{hr}$ at $4^{\circ} \mathrm{C}$. The lysates and beads were then washed thrice with lysis buffer and eluted in SDS lysis buffer for 5 minutes at $95^{\circ} \mathrm{C}$ and resolved by loading equal amounts of eluted protein on a Mini-Protean TGX (4-20\%) SDS-PAGE (Bio-rad). The separated proteins were transferred onto polyvinylidene difluoride membrane (PVDF, Bio-Rad), and blocked for 1 hour with Odyssey Blocking Buffer (PBS). Membranes were then incubated overnight at $4^{\circ} \mathrm{C}$ with phospho-T172 AMPK or total AMPK primary antibodies diluted in the same blocking buffer. Incubations with Li-Cor fluorescence-coupled secondary antibodies were performed for 1 hour at room temperature, and visualization was performed by Li-Cor Odyssey Blot Imager.

\section{Analysis of Spine Density and Mitochondrial Morphology}

Dendritic spine densities were estimated on secondary or tertiary dendritic branches for cultured neurons and in the depth of the $z$ stack for slices, using FiJi software (ImageJ, NIH). The length of the dendritic segment was measured on the $z$ projection, which implies the density could be overestimated. To limit this issue, only dendrites that were parallel to the plane of the slices were analyzed. Spines were quantified over an average of $40 \mu \mathrm{m}$ in

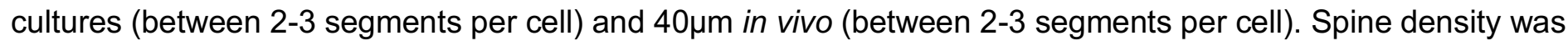
defined as the number of quantified spines divided by the length over which the spines were quantified. The criteria for measuring mitochondrial length for each dendritic segment were same as above. Mitochondrial density was defined as the total sum of all the mitochondria divided by the length over which the mitochondria lengths were measured.

\section{Analysis of Mitophagy Events}

For Fig.2B, manual counting of total number of dendrites and number of dendrites showing accumulation of fluorescently-tagged LC3-RFP and LAMP1-mEmerald was performed for all imaged neurons. To confirm both LC3 and LAMP1 accumulation over time following A 420 application using an independent quantification approach, we selectively examined all of the 251 dendritic segments categorized as showing LC3+/LAMP1+ accumulation upon A 3420 application and measured the intensities of LC3 and LAMP1 fluorescence (within an ROI ranging from $20-30 \mu \mathrm{m}$ in length) over time. All the dendrites that showed accumulation of LC3 and LAMP1 in control conditions were also analyzed, as well as randomly selected dendritic segments that did not show detectable accumulation of LC3 and LAMP1. Optical density of LAMP1-mEmerald and LC3-RFP fluorescence were normalized to the initial starting fluorescence intensities at $t_{0}\left(\Delta F / F_{0}\right)$. For these selected dendrites, mitomtagBFP2 intensities were also measured over time for Fig. 2E. To measure local mitophagy events as indicated in Fig. 2F, random dendrites analyzed in Fig.2B were selected for each condition. For a given dendritic segment, intensity line scan analysis (pixel neighborhood-1 pixel) was performed where two vertical lines were drawn: one along the region of dendrite without LC3/LAMP1 puncta and one along the region with LC3/LAMP1 puncta. The fold change of mitochondrial intensity from time point zero to $14 \mathrm{hr}$ after respective treatment was calculated as illustrated in Fig. 2F.

\section{Analysis of Mitochondrial Density in hES cell-derived cortical neurons}

For Figure S7B-C, lines of equal thickness and of an average length of $\sim 40 \mu \mathrm{m}$ were drawn along the neurites of differentiated hES cell-derived cortical neurons in Nikon NIS-Elements Software. A kymograph was subsequently created from each drawn line, for which the mean fluorescence intensity of the mitochondria as a function of area was calculated and normalized to the background fluorescence intensity of the image.

\section{References}


Courchet, J., Lewis, T.L., Jr., Lee, S., Courchet, V., Liou, D.Y., Aizawa, S., and Polleux, F. (2013). Terminal axon branching is regulated by the LKB1-NUAK1 kinase pathway via presynaptic mitochondrial capture. Cell 153, 1510-1525.

Lewis, T.L., Jr., Turi, G.F., Kwon, S.K., Losonczy, A., and Polleux, F. (2016). Progressive Decrease of Mitochondrial Motility during Maturation of Cortical Axons In Vitro and In Vivo. Curr Biol 26, 2602-2608.

Szczurkowska, J., Cwetsch, A.W., dal Maschio, M., Ghezzi, D., Ratto, G.M., and Cancedda, L. (2016). Targeted in vivo genetic manipulation of the mouse or rat brain by in utero electroporation with a triple-electrode probe. Nat Protoc 11, 399-412.

Toyama, E., Herzig, S., Courchet, J., Lewis Jr., T., Loson, O., Hellberg, K., Young, N., Chen, H., Polleux, F., Chan, D.C., et al. (2016). AMP-activated protein kinase mediates mitochondrial fission in response to energy stress. Science 351, 275-281.

Zhou, X., Babu, J.R., da Silva, S., Shu, Q., Graef, I.A., Oliver, T., Tomoda, T., Tani, T., Wooten, M.W., and Wang, F. (2007). Unc-51-like kinase 1/2-mediated endocytic processes regulate filopodia extension and branching of sensory axons. Proc Natl Acad Sci U S A 104, 5842-5847. 